Article

\title{
Cu-Catalyzed Arylation of Bromo-Difluoro-Acetamides by Aryl Boronic Acids, Aryl Trialkoxysilanes and Dimethyl-Aryl-Sulfonium Salts: New Entries to Aromatic Amides
}

\author{
Satenik Mkrtchyan 1,*(D), Michał Jakubczyk ${ }^{1,2} \mathbb{D}^{\mathbb{D}}$, Suneel Lanka ${ }^{1,3} \mathbb{D}^{\mathbb{D}}$, Michael Pittelkow ${ }^{4}(\mathbb{D})$ \\ and Viktor O. Iaroshenko $1,5,6, *$ (D)
}

check for updates

Citation: Mkrtchyan, S.; Jakubczyk, M.; Lanka, S.; Pittelkow, M.; Iaroshenko, V.O. Cu-Catalyzed Arylation of

Bromo-Difluoro-Acetamides by Aryl Boronic Acids, Aryl Trialkoxysilanes and Dimethyl-Aryl-Sulfonium Salts: New Entries to Aromatic Amides. Molecules 2021, 26, 2957. https:// doi.org/10.3390/molecules26102957

Academic Editor: Norio Shibata

Received: 30 April 2021

Accepted: 14 May 2021

Published: 16 May 2021

Publisher's Note: MDPI stays neutral with regard to jurisdictional claims in published maps and institutional affiliations.

Copyright: (c) 2021 by the authors. Licensee MDPI, Basel, Switzerland. This article is an open access article distributed under the terms and conditions of the Creative Commons Attribution (CC BY) license (https:// creativecommons.org/licenses/by/ $4.0 /)$.
1 Laboratory of Homogeneous Catalysis and Molecular Design at the Center of Molecular and Macromolecular Studies, Polish Academy of Sciences, Sienkiewicza 112, 90-363 Łodź, Poland; drmjakubczyk@gmail.com (M.J.); suneellanka89@gmail.com (S.L.)

2 Institute of Bioorganic Chemistry, Polish Academy of Sciences, Noskowskiego 12/14, 61-704 Poznań, Poland

3 Institute of General and Ecological Chemistry, Lodz University of Technology, Zeromskiego 116, 90-924 Lodz, Poland

4 Department of Chemistry, University of Copenhagen, Universitetsparken 5, 2100 Copenhagen, Denmark; pittel@chem.ku.dk

5 Dipartimento di Chimica e Biologia “A. Zambelli” Università di Salerno, Via Giovanni Paolo II, 84084 Fisciano (SA), Italy

6 Department of Chemistry, University of Helsinki, A.I. Virtasen aukio 1, 00014 Helsinki, Finland

* Correspondence: sm19860214@googlemail.com (S.M.); yva108@yahoo.co.uk (V.O.I.)

Abstract: We describe a mechanism-guided discovery of a synthetic methodology that enables the preparation of aromatic amides from 2-bromo-2,2-difluoroacetamides utilizing a copper-catalyzed direct arylation. Readily available and structurally simple aryl precursors such as aryl boronic acids, aryl trialkoxysilanes and dimethyl-aryl-sulfonium salts were used as the source for the aryl substituents. The scope of the reactions was tested, and the reactions were insensitive to the electronic nature of the aryl groups, as both electron-rich and electron-deficient aryls were successfully introduced. A wide range of 2-bromo-2,2-difluoroacetamides as either aliphatic or aromatic secondary or tertiary amides were also reactive under the developed conditions. The described synthetic protocols displayed excellent efficiency and were successfully utilized for the expeditious preparation of diverse aromatic amides in good-to-excellent yields. The reactions were scaled up to gram quantities.

Keywords: fluorine; amides; copper; catalysis; C-C-coupling; boronic acids; aryl trialkoxysilanes; dimethyl-aryl-sulfonium salts

\section{Introduction}

The amide functional group is abundant in peptides and numerous natural products and is also ubiquitous in a vast range of biologically active compounds, marketed drugs, and a broad spectrum of agrochemicals [1-7]. The presence of the amide motif or its isosteres condition biological activity of many privileged scaffolds [7]. By recent estimates, almost a quarter of all marketed pharmaceuticals possesses an amide bond, making this functional group the most encountered in medicinal chemistry. Amides are prevalent in advanced materials [7,8], and many life science relevant substances; amides also play pivotal roles in supramolecular chemistry [9,10], molecular recognition [9-11], and catalysis $[12,13]$. The amide functional group can be tuned electronically and conformationally to gain desired structural, physical, and biological properties. The chemistry of amide group is vast, and by its virtue amides can be transformed into many other functional groups [14-20]. Due to the omnipresence and profound importance of the amide functionality, the development of principally new synthetic routes aiming at installation of the 
amide structural moiety is of current importance in both modern organic and medicinal chemistry. In this context, many new synthetic routes were elaborated [21-24]. Among those, it is important to mention such game-changing strategy as aminocarbonylations of aryl halides utilizing $\mathrm{CO}$ [25-27].

One conceptually underexplored strategy to prepare new amides was the installation of the amide structural unit by the attaching an appropriate substituent onto the prefunctionalized $\mathrm{CO}-\mathrm{N}$ structural motif bearing a tuned leaving group on the amide carbon. Analysis of the literature revealed that this tactic has been realized using $\mathrm{C}-\mathrm{N}$ synthons bearing $\mathrm{Cl}$ [28] and $\mathrm{CHal}_{3}(\mathrm{Hal}=\mathrm{Cl}, \mathrm{Br}, \mathrm{I})$ as a leaving group [29-31]. These strategies were predominately used for the construction of aromatic amides with different substituents on the nitrogen atom. Another method was developed that is based upon the transition-metalcatalyzed arylation of N-substituted formamides by different aryl-containing reagents, predominantly aryl halides [32-34].

Based on a mechanistic consideration, we considered that 2-bromo-2,2-difluoroacetamides 1 would be particularly attractive for the formation of aryl-amides by activation using transition-metal catalysis. Combining the halogens in this particular fashion on the trihaloacetamide enables us to harness the attractive features of copper catalysis and fluoride-mediated catalysis. We set out to explore 2-bromo-2,2-difluoroacetamides 1 in coupling reactions with aryl boronic acids 2 and (aryl)trialkoxysilanes 3 arylation agents as donors of aryl or heteroaryl substituents (Scheme 1a). We hypothesized (Scheme 1b) that using transition-metal-assisted catalysis, a 2-bromo-2,2-difluoroacetamide unit could undergo an oxidative addition on an appropriately tuned by ligands metal nuclei, forming an organometallic intermediate (structure 6) [35-37], followed by a rearrangement possibly via a $\mathrm{CF}_{2}$-carbene complex 7 , which undergoes loss of difluorocarben and simultaneous exchange of $\mathrm{Br}$ versus $\mathrm{F}$ giving rise to an organometallic (intermediate 8) capable of undergoing reaction with aryl boronic acids or aryl trialkoxysilanes to deliver a new intermediate (9), which after the reductive elimination would result in the formation of a new $\mathrm{C}-\mathrm{C}$ bond to yield the desired aryl amide (5). An alternative mechanistic pathway could be via copper-intermediate $\mathbf{1 1}$ (Scheme 1c), as a result of the reaction between a fluorinated transition-metal catalyst and an aryl boronic acid (or aryl trialkoxysilane). This species could react with a carbon-centered radical 10 to form the species $\mathbf{9 ,}$ which then decomposes into the final amide product $\mathbf{5}$. The formation of the radical species $\mathbf{1 0}$ would be unusual from the mechanistic point of view. A similar mechanism has, however, been suggested on the instance of palladium-catalyzed carboxylate-assisted ethoxycarboxylation of aromatic acids by ethyl bromodifluoroacetate in a very recent study [38,39]. It is worth noting that the concept of $\mathrm{F}$ versus $\mathrm{B}(\mathrm{OR})_{2}$ (or $\mathrm{Si}(\mathrm{OR})_{3}$ ) exchange on the copper nuclei, which we are postulating here, was suggested by Giri and Brawn for the mechanism in their copper-catalyzed Suzuki-Miyaura C-C couplings. These protocols were operational not only for boronic esters, but also for a broad range of trialkoxysilanes [40-42]. Based on the assumption of a fluoride-bearing Ar-Cu-F intermediate being active (similar to structure 8), and in a view of the resent literature on copper-supported $C-C$ coupling protocols, we envisioned the use of copper catalysts. We also envisioned the preparation of aromatic amides as a result of the C-C coupling between aryl boronic acids, aryl trialkoxysilanes, or sulphonium salts with 2-bromo-2,2-difluoroacetamides according to the general synthetic scenario depicted in the Scheme 1. 

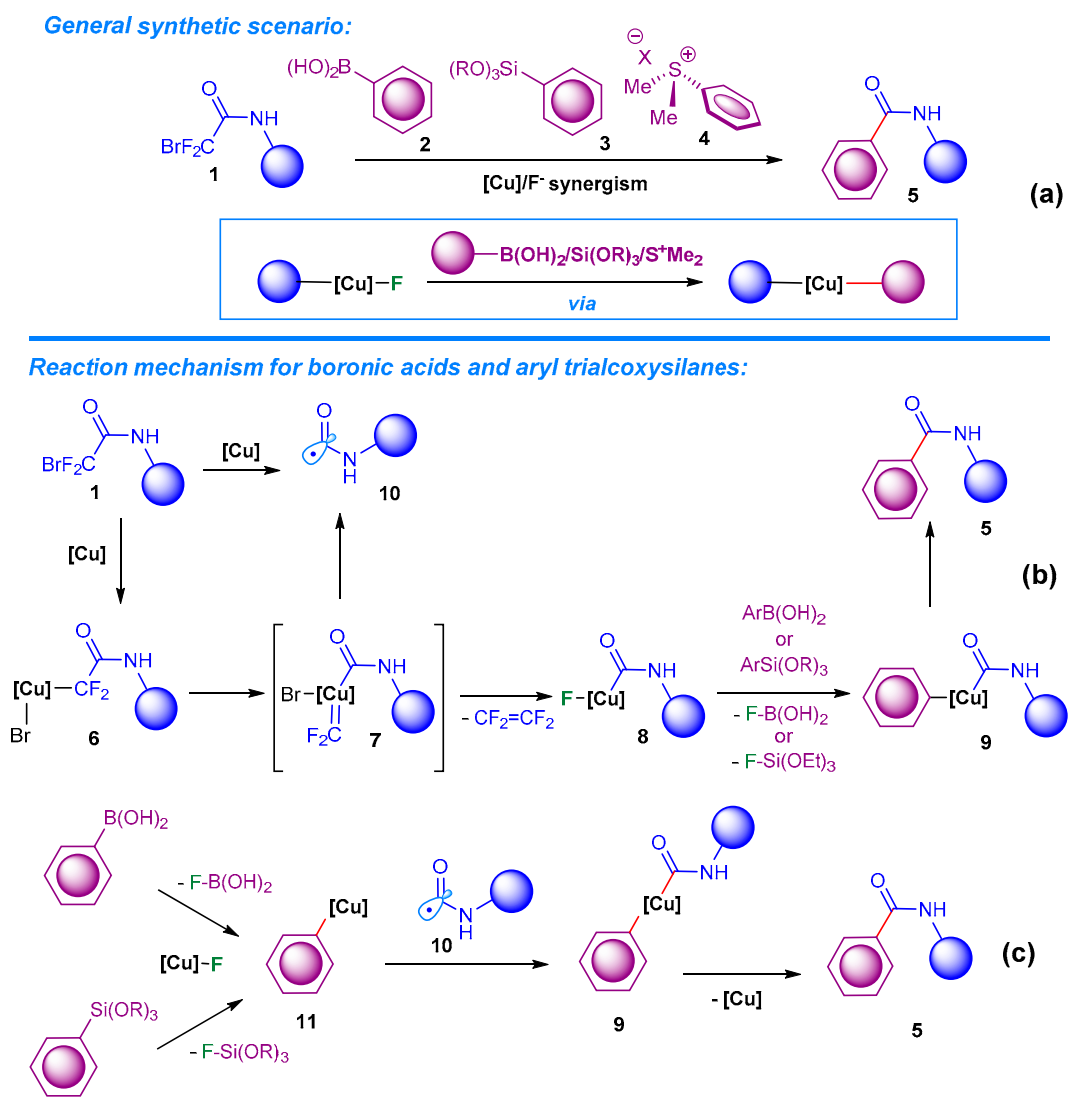

Scheme 1. (a) Synthetic scenario, (b,c) Proposed reaction mechanisms.

We first considered the use of 2-bromo-2,2-difluoroacetamides as a source of the -CO$\mathrm{NR}_{2}$ synthon. The only literature example known to date where ethoxycarboxylation of aromatic acids occurs using ethyl bromodifluoroacetate was described recently by Zhao et al. [38]. Similar access was proposed by Shi and co-workers in an alkoxycarbonylation of benzamides utilizing chloroformates [28]. Trifluoroacetyl amides have been used for the construction of aromatic and aliphatic amides via $\mathrm{C}(\mathrm{O})-\mathrm{CF}_{3}$ bond cleavage utilizing the reaction with Grignard reagents [43]. The routes proposed by us utilize commercially or readily available reagents aryl donors and are visibly more atom economic and efficient than those using metalorganic reagents, thus enabling the creation larger amide structural diversities.

\section{Results and Discussion}

We selected three model reactions and performed a set of trial experiments to identify the trends and generalities depicted in Scheme 2 and Tables 1-3. After testing numerous reaction parameters, among which are catalysts, ligands, solvents, and bases, we noticed that some of the copper salts in combination with nitrogen-containing ligands (not indicated in the optimization Tables), in particular solvents, facilitate the expected C-C-coupling reaction and thus the formation of the desired aromatic amide. Furthermore, we succeeded in establishing the optimal reaction conditions for synthetic protocols (a) and (b), which were identical and consisted in the use of $\mathrm{CuBr}_{2}$ (0.1 equiv.), $\mathrm{KF}$ (2 equiv.), $\mathrm{MgCl}_{2}$ (1 equiv.) with hexafluoropropanol as the solvent, where all reactions were conducted in ACE pressure tubes at $70{ }^{\circ} \mathrm{C}$ for $8 \mathrm{~h}$. One crucial aspect appeared to be the addition of calix[4]arene derivatives, which most probably act as ligands for the coper salt. The best efficiency was observed for the corresponding calix[4]arene L1. The magnesium salt, due to the high affinity of $\mathrm{Mg}^{2+}$ towards electron rich fluoride ion (hardness of $\mathrm{Mg}^{2+}$ in terms of the Pearson Hard-Soft acid-base theory), is most probably involved in the activation of one of the C-Hal bonds, like the corresponding C-F bond, by the coordination onto fluorine 
(where the fluoride ion in turn is a hard base, as per the Pearson Hard-Soft acid-base theory) and formation of the Mg-haloalkane complex [44,45]. The optimized reaction conditions allowed the efficient preparation of the model amide compound $\mathbf{5 a}$ in $87 \%$ and $90 \%$, respectively (Tables 1 and 2). This success encouraged further exploration of the scope and limitation of these two new protocols. We set out to test the scope and limitations of these coupling reactions by selecting twenty-two 2-bromo-2,2-difluoroacetamides 1 and reacting those with a range of aryl boronic acids 2 (twenty-three different substrates) and aryl trialkoxysilanes 3 (seventeen substrates). In a result of this study, we successfully prepared thirty-one amide derivativities 5 in good-to-excellent yields.

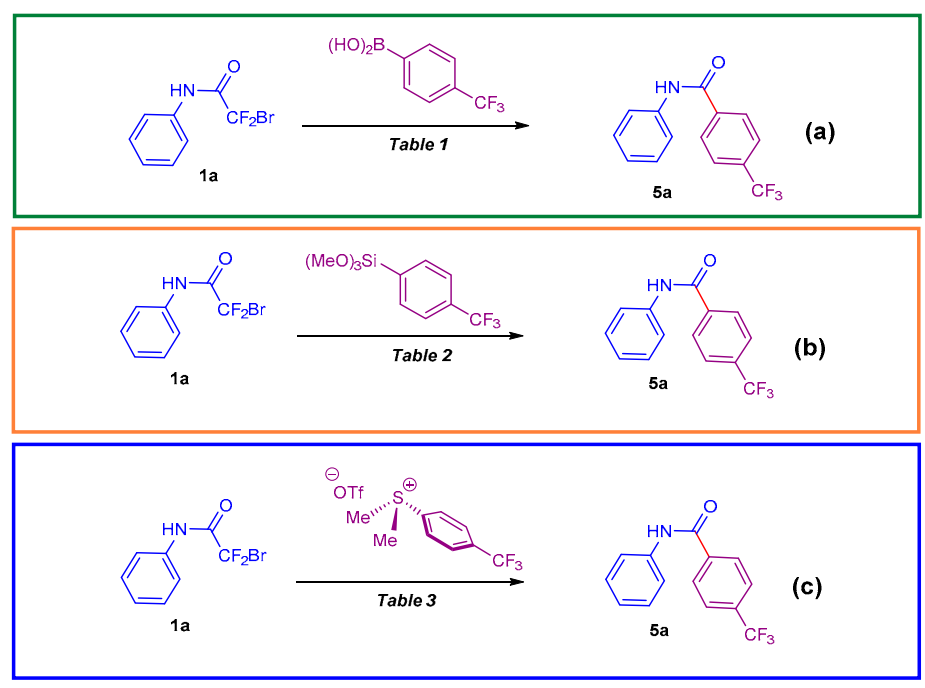

Scheme 2. Model reactions for reaction conditions optimization: (a) Reaction of 2-bromo-2,2-difluoro$\mathrm{N}$-phenylacetamide with (4-(trifluoromethyl)phenyl)boronic acid, (b) Reaction of 2-bromo-2,2-difluoro$N$-phenylacetamide with trimethoxy(4-(trifluoromethyl)phenyl)silane, (c) Reaction of 2-bromo-2,2difluoro- $N$-phenylacetamide with dimethyl(4-(trifluoromethyl)phenyl)sulfonium triflate.

Focusing first on the reactions utilizing aryl boronic acids and aryl trialkoxysilanes, these synthetic protocols were tolerant to numerous functional groups placed on both coupling partners. In particular, both methodologies allowed the coupling of aryl substrates bearing a vast range of electron-withdrawing and electron-donating substituents placed in ortho-, meta-, and para- positions, respectively; among those are alkyl groups, alkoxy groups, $\mathrm{Ph}$, halogens including fluorine, as well as $\mathrm{CF}_{3}, \mathrm{CF}_{3} \mathrm{O}$, and $\mathrm{CF}_{3} \mathrm{~S}$ groups. Substrates bearing 1-naphthyl, 1-thiophenyl, and 3-pyridyl moieties also showed excellent efficiency with some discrepancy for the formation of the thionyl derivative $5 \mathbf{n}$ (Scheme 3). Interestingly, both protocols were operational for aryl substrates bearing diverse ortho substituents $\left(\mathrm{Me}, \mathrm{F}, \mathrm{Cl}, \mathrm{Br}, \mathrm{CF}_{3}, \mathrm{CF}_{3} \mathrm{O}\right.$ ). Of note, highly fluorinated boronic acids and aryl trialkoxysilanes were prone to enter those protocols readily delivering the corresponding amides 5g, 5o, 5q. Regarding the reactivity of 2-bromo-2,2-difluoroacetamide counterparts 1, we did not observe any influence on the reaction efficiency of the substituents placed on the amide nitrogen - both alkyl and aryl groups as well as mixed derivatives exerted excellent tolerability within the developed protocols (Scheme 3). These reactions were not affected by changing a substitution pattern on the 2-bromo-2,2-difluoroacetamides: Species with alkyl as well as aryl substituents on the amide motif were equally effective within both synthetic protocols (Scheme 2). To further demonstrate the synthetic utility of these methodologies, the gram-scale reactions were successfully performed using $10 \mathrm{mmol}$ of the 2-bromo-2,2-difluoroacetamides, which yielded the expected products in high yields. 
Table 1. Optimization of the reaction conditions for synthetic protocol (a).

\begin{tabular}{|c|c|c|c|}
\hline \multicolumn{4}{|c|}{ Reaction (a) $^{b}$} \\
\hline Entry & Reagent (Equiv)/Catalyst/Ligand/Additive & Solvent/Temperature/Time & Yield (\%) $5 a^{a}$ \\
\hline 1 & boronic acid (1.5)/CuI (0.1)/KF (2.0) & $\mathrm{DMF} / 100^{\circ} \mathrm{C} / 12 \mathrm{~h}$ & 0 \\
\hline 2 & boronic acid (1.5)/ $\mathrm{CuBr}_{2}(0.1) / \mathrm{KF}(2.0)$ & $\mathrm{DMF} / 100^{\circ} \mathrm{C} / 12 \mathrm{~h}$ & Trace \\
\hline 3 & $\begin{array}{c}\text { boronic acid (1.5)/CuI (0.1)/KF (2.0), } \mathrm{MgCl}_{2} \\
\text { (1.0) }\end{array}$ & $\left(\mathrm{CF}_{3}\right)_{2} \mathrm{CHOH} / 70^{\circ} \mathrm{C} / 12 \mathrm{~h}$ & 12 \\
\hline 4 & $\begin{array}{c}\text { boronic acid (1.5)/CuI (0.1)/L1 (0.2)/KF (2.0), } \\
\mathrm{MgCl}_{2}(1.0)\end{array}$ & $\left(\mathrm{CF}_{3}\right)_{2} \mathrm{CHOH} / 70^{\circ} \mathrm{C} / 12 \mathrm{~h}$ & 38 \\
\hline 5 & $\begin{array}{c}\text { boronic acid (1.5)/ } \mathrm{CuF}_{2}(0.1) / \mathrm{L} 1(0.2) / \mathrm{KF} \\
\text { (2.0), } \mathrm{MgCl}_{2}(1.0)\end{array}$ & $\left(\mathrm{CF}_{3}\right)_{2} \mathrm{CHOH} / 70^{\circ} \mathrm{C} / 12 \mathrm{~h}$ & 49 \\
\hline 6 & $\begin{array}{c}\text { boronic acid (1.5)/ } \mathrm{CuCl}_{2}(0.1) / \mathrm{L} 1(0.2) / \mathrm{KF} \\
(2.0), \mathrm{MgCl}_{2}(1.0)\end{array}$ & $\left(\mathrm{CF}_{3}\right)_{2} \mathrm{CHOH} / 70^{\circ} \mathrm{C} / 12 \mathrm{~h}$ & 67 \\
\hline 7 & $\begin{array}{c}\text { boronic acid (1.5)/ } \mathrm{CuBr}_{2}(0.1) / \mathrm{L} 1 \text { (0.2)/KF } \\
\text { (2.0), } \mathrm{MgCl}_{2}(1.0)\end{array}$ & $\left(\mathrm{CF}_{3}\right)_{2} \mathrm{CHOH} / 70^{\circ} \mathrm{C} / 12 \mathrm{~h}$ & 88 \\
\hline 8 & $\begin{array}{c}\text { boronic acid (1.3)/CuBr} 2(0.1) / \mathrm{L} 1(0.2) / \mathrm{KF} \\
\text { (2.0), } \mathrm{MgCl}_{2}(1.0)\end{array}$ & $\left(\mathrm{CF}_{3}\right)_{2} \mathrm{CHOH} / 70^{\circ} \mathrm{C} / 8 \mathrm{~h}$ & 87 \\
\hline 9 & $\begin{array}{c}\text { boronic acid (1.3)/ } \mathrm{CuBr}_{2}(0.1) / \mathrm{L} 1(0.2) / \mathrm{KF} \\
\text { (2.0), } \mathrm{MgCl}_{2}(1.0)\end{array}$ & $\mathrm{DMF} / 70^{\circ} \mathrm{C} / 8 \mathrm{~h}$ & 11 \\
\hline 10 & $\begin{array}{c}\text { boronic acid (1.3)/ } \mathrm{CuBr}_{2}(0.1) / \mathrm{KF}(2.0) \text {, } \\
\qquad \mathrm{MgCl}_{2}(1.0)\end{array}$ & $\left(\mathrm{CF}_{3}\right)_{2} \mathrm{CHOH} / 70^{\circ} \mathrm{C} / 8 \mathrm{~h}$ & 18 \\
\hline 11 & $\begin{array}{c}\text { boronic acid (1.3)/L1 (0.2)/KF (2.0), } \mathrm{MgCl}_{2} \\
\text { (1.0) }\end{array}$ & $\left(\mathrm{CF}_{3}\right)_{2} \mathrm{CHOH} / 70^{\circ} \mathrm{C} / 8 \mathrm{~h}$ & 0 \\
\hline 12 & $\begin{array}{c}\text { boronic acid (1.3)/ } \mathrm{CuBr}_{2}(0.1) / \mathrm{L} 1(0.2) / \mathrm{KF} \\
\qquad(2.0)\end{array}$ & $\left(\mathrm{CF}_{3}\right)_{2} \mathrm{CHOH} / 70^{\circ} \mathrm{C} / 8 \mathrm{~h}$ & 27 \\
\hline 13 & $\begin{array}{c}\text { boronic acid (1.3)/ } \mathrm{CuBr}_{2}(0.1) / \mathrm{L} 1(0.2) / \mathrm{KF} \\
(2.0), \mathrm{MgCl}_{2}(1.0) / \mathrm{TEMPO}(2.0)\end{array}$ & $\left.\mathrm{CF}_{3}\right)_{2} \mathrm{CHOH} / 70^{\circ} \mathrm{C} / 8 \mathrm{~h}$ & 75 \\
\hline 14 & $\begin{array}{c}\text { boronic acid (1.3)/ } \mathrm{CuBr}_{2}(0.1) / \mathrm{L} 1(0.2) / \mathrm{KF} \\
(2.0), \mathrm{MgCl}_{2}(1.0) / \mathrm{TEMPO}(3.0)\end{array}$ & $\left(\mathrm{CF}_{3}\right)_{2} \mathrm{CHOH} / 70^{\circ} \mathrm{C} / 8 \mathrm{~h}$ & 60 \\
\hline 15 & $\begin{array}{c}\text { boronic acid (1.3)/ } \mathrm{CuBr}_{2}(0.1) / \mathrm{L} 1(0.2) / \mathrm{KF} \\
(2.0), \mathrm{MgCl}_{2}(1.0) \text { - in dark }\end{array}$ & $\left(\mathrm{CF}_{3}\right)_{2} \mathrm{CHOH} / 70^{\circ} \mathrm{C} / 8 \mathrm{~h}$ & 84 \\
\hline
\end{tabular}

a Isolated yield. ${ }^{\mathrm{b}}$ All reactions were conducted in inert atmosphere.

Table 2. Optimization of the reaction conditions for synthetic protocol (b).

\begin{tabular}{|c|c|c|c|}
\hline \multicolumn{4}{|c|}{ Reaction (b) $b$} \\
\hline Entry & Reagent (Equiv)/Catalyst/Ligand/Additive & Solvent/Temperature/Time & Yield (\%) $5 \mathrm{a}^{\mathrm{a}}$ \\
\hline 1 & $\begin{array}{l}\text { aryl trialkoxysilane }(1.4) / \mathrm{CuBr}_{2}(0.1) / \mathrm{L} 1 \\
(0.2) / \mathrm{KF}(2.0), \mathrm{MgCl}_{2}(1.0)\end{array}$ & $\left(\mathrm{CF}_{3}\right)_{2} \mathrm{CHOH} / 70^{\circ} \mathrm{C} / 8 \mathrm{~h}$ & 90 \\
\hline 2 & $\begin{array}{l}\text { aryl trialkoxysilane (1.4)/ } / \mathrm{CuBr}_{2}(0.1) / \mathrm{L} 1 \\
(0.2) / \mathrm{KF}(2.0), \mathrm{MgCl}_{2}(1.0)\end{array}$ & $\mathrm{DMF} / 70^{\circ} \mathrm{C} / 8 \mathrm{~h}$ & 22 \\
\hline 3 & $\begin{array}{l}\text { aryl trialkoxysilane }(1.4) / \mathrm{CuBr}_{2}(0.1) / \mathrm{KF} \\
\text { (2.0), } \mathrm{MgCl}_{2}(1.0)\end{array}$ & $\left(\mathrm{CF}_{3}\right)_{2} \mathrm{CHOH} / 70^{\circ} \mathrm{C} / 8 \mathrm{~h}$ & 21 \\
\hline 4 & $\begin{array}{l}\text { aryl trialkoxysilane (1.4)/L1 (0.2)/KF (2.0), } \\
\qquad \mathrm{MgCl}_{2}(1.0)\end{array}$ & $\left(\mathrm{CF}_{3}\right)_{2} \mathrm{CHOH} / 70^{\circ} \mathrm{C} / 8 \mathrm{~h}$ & 0 \\
\hline 5 & $\begin{array}{l}\text { aryl trialkoxysilane }(1.4) / \mathrm{CuBr}_{2}(0.1) / \mathrm{L} 1 \\
(0.2) / \mathrm{KF}(2.0)\end{array}$ & $\left(\mathrm{CF}_{3}\right)_{2} \mathrm{CHOH} / 70^{\circ} \mathrm{C} / 8 \mathrm{~h}$ & 25 \\
\hline 6 & $\begin{array}{l}\text { aryl trialkoxysilane (1.4)/CuBr }(0.1) / \mathrm{L} 1 \\
(0.2) / \mathrm{KF}(2.0), \mathrm{MgCl}_{2}(1.0) / \mathrm{TEMPO}(2.0)\end{array}$ & $\left(\mathrm{CF}_{3}\right)_{2} \mathrm{CHOH} / 70^{\circ} \mathrm{C} / 8 \mathrm{~h}$ & 72 \\
\hline
\end{tabular}


Table 2. Cont.

\begin{tabular}{cccc}
\hline \multicolumn{2}{c}{ Reaction $(\mathbf{b}) \mathbf{b}$} & \\
Entry & Reagent (Equiv)/Catalyst/Ligand/Additive & Solvent/Temperature/Time & Yield (\%) 5a ${ }^{\mathbf{a}}$ \\
\hline 7 & $\begin{array}{c}\text { aryl trialkoxysilane }(1.4) / \mathrm{CuBr}_{2}(0.1) / \mathrm{L} 1 \\
(0.2) / \mathrm{KF}(2.0), \mathrm{MgCl}_{2}(1.0) / \mathrm{TEMPO}(3.0)\end{array}$ & $\left(\mathrm{CF}_{3}\right)_{2} \mathrm{CHOH} / 70{ }^{\circ} \mathrm{C} / 8 \mathrm{~h}$ & 58 \\
\hline \multirow{2}{*}{8} & $\begin{array}{c}\text { aryl trialkoxysilane }(1.4) / \mathrm{CuBr}_{2}(0.1) / \mathrm{L} 1 \\
(0.2) / \mathrm{KF}(2.0), \mathrm{MgCl}_{2}(1.0) \text {-in dark }\end{array}$ & $\left(\mathrm{CF}_{3}\right)_{2} \mathrm{CHOH} / 70{ }^{\circ} \mathrm{C} / 8 \mathrm{~h}$ & 91 \\
\hline
\end{tabular}

${ }^{\mathrm{a}}$ Isolated yield. ${ }^{\mathrm{b}}$ All reactions were conducted at room temperature in inert atmosphere.

Table 3. Optimization of the reaction conditions for synthetic protocol (c).

\begin{tabular}{|c|c|c|c|}
\hline Entry & Reagent (Equiv)/Catalyst/Ligand/Additive & Solvent/Temperature/ Time & Yield $(\%) 5 a^{a}$ \\
\hline 1 & $\begin{array}{c}\text { sulphonium salt (1.4)/ } / \mathrm{CuBr}_{2}(0.1) / \mathrm{L} 1 \\
(0.2) / \mathrm{KF}(2.0), \mathrm{MgCl}_{2}(1.0)\end{array}$ & $\left(\mathrm{CF}_{3}\right)_{2} \mathrm{CHOH} / 70^{\circ} \mathrm{C} / 12 \mathrm{~h}$ & 47 \\
\hline 2 & $\begin{array}{l}\text { sulphonium salt (2)/ } \mathrm{CuCl}_{2}(0.1), / \mathrm{L} 1 \\
(0.2) / \mathrm{KF}(2.0), \mathrm{MgCl}_{2}(1.0)\end{array}$ & $\left(\mathrm{CF}_{3}\right)_{2} \mathrm{CHOH} / 70{ }^{\circ} \mathrm{C} / 12 \mathrm{~h}$ & Trace \\
\hline 3 & $\begin{array}{l}\text { sulphonium salt (2)/CuI (0.1), / } \mathrm{L} 1 \text { (0.2)/KF } \\
\qquad(2.0), \mathrm{MgCl}_{2}(1.0)\end{array}$ & $\left(\mathrm{CF}_{3}\right)_{2} \mathrm{CHOH} / 70^{\circ} \mathrm{C} / 12 \mathrm{~h}$ & Trace \\
\hline 4 & $\begin{array}{l}\text { sulphonium salt (1.6)/ } \mathrm{CuBr}_{2}(0.3), \mathrm{Pd}(\mathrm{OAc})_{2} \\
(0.2) / \mathrm{L} 1(0.25) / \mathrm{KF}(2.0), \mathrm{MgCl}_{2}(1.0)\end{array}$ & $\left(\mathrm{CF}_{3}\right)_{2} \mathrm{CHOH} / 70^{\circ} \mathrm{C} / 12 \mathrm{~h}$ & 17 \\
\hline 5 & $\begin{array}{l}\text { sulphonium salt (1.6)/ } \mathrm{CuBr}_{2}(0.3) / \mathrm{L} 2 \\
(0.25) / \mathrm{KF}(2.0), \mathrm{MgCl}_{2}(1.0)\end{array}$ & $\left(\mathrm{CF}_{3}\right)_{2} \mathrm{CHOH} / 70^{\circ} \mathrm{C} / 12 \mathrm{~h}$ & 29 \\
\hline 6 & $\begin{array}{l}\text { sulphonium salt (1.6)/ } \mathrm{CuBr}_{2}(0.3), \mathrm{PdCl}_{2} \\
\quad(0.2) / \mathrm{L} 2(0.25) / \mathrm{KF}(2.0), \mathrm{MgCl}_{2}(1.0)\end{array}$ & $\left(\mathrm{CF}_{3}\right)_{2} \mathrm{CHOH} / 70^{\circ} \mathrm{C} / 12 \mathrm{~h}$ & 48 \\
\hline 7 & $\begin{array}{l}\text { sulphonium salt }(1.6) / \mathrm{CuBr}_{2}(0.3), \mathrm{Pd}(\mathrm{OAc})_{2} \\
(0.2) / \mathrm{L} 2(0.25) / \mathrm{KF}(2.0), \mathrm{MgCl}_{2}(1.0)\end{array}$ & $\left(\mathrm{CF}_{3}\right)_{2} \mathrm{CHOH} / 70^{\circ} \mathrm{C} / 12 \mathrm{~h}$ & 53 \\
\hline 8 & $\begin{array}{c}\text { sulphonium salt (1.6)/CuBr}{ }_{2}(0.3) \\
{\left[\mathrm{Ru}\left(\mathrm{p} \text {-cymene) } \mathrm{Cl}_{2}\right]_{2}(0.2) / \mathrm{L} 2(0.25) / \mathrm{KF}(2.0)\right.} \\
\mathrm{MgCl}_{2}(1.0)\end{array}$ & $\left(\mathrm{CF}_{3}\right)_{2} \mathrm{CHOH} / 70^{\circ} \mathrm{C} / 11 \mathrm{~h}$ & 84 \\
\hline 9 & $\begin{array}{c}\text { sulphonium salt }(1.6) / \mathrm{CuBr}_{2}(0.3), \\
{\left[\mathrm{Ru}(\mathrm{p} \text {-cymene }) \mathrm{Cl}_{2}\right]_{2}(0.2) / \mathrm{KF}(2.0), \mathrm{MgCl}_{2}} \\
(1.0)\end{array}$ & $\left(\mathrm{CF}_{3}\right)_{2} \mathrm{CHOH} / 70{ }^{\circ} \mathrm{C} / 11 \mathrm{~h}$ & 27 \\
\hline 10 & $\begin{array}{l}\left.\text { sulphonium salt (1.6)/[Ru(p-cymene) } \mathrm{Cl}_{2}\right]_{2} \\
\quad(0.2) / \mathrm{L} 2(0.25) / \mathrm{KF}(2.0), \mathrm{MgCl}_{2}(1.0)\end{array}$ & $\left(\mathrm{CF}_{3}\right)_{2} \mathrm{CHOH} / 70^{\circ} \mathrm{C} / 11 \mathrm{~h}$ & 0 \\
\hline 11 & $\begin{array}{c}\text { sulphonium salt (1.6)/ } \mathrm{CuBr}_{2}(0.3) \\
{\left[\mathrm{Ru}(\mathrm{p} \text {-cymene }) \mathrm{Cl}_{2}\right]_{2}(0.2) / \mathrm{L} 2(0.25) / \mathrm{KF}(2.0)}\end{array}$ & $\left(\mathrm{CF}_{3}\right)_{2} \mathrm{CHOH} / 70^{\circ} \mathrm{C} / 11 \mathrm{~h}$ & 18 \\
\hline 12 & $\begin{array}{c}\text { sulphonium salt (1.6)/CuBr} 2(0.3) \\
{\left[\begin{array}{c}\left.\mathrm{Ru}(\mathrm{p} \text {-cymene }) \mathrm{Cl}_{2}\right]_{2}(0.2) / \mathrm{L} 2(0.25) / \mathrm{KF}(2.0) \\
\mathrm{MgCl}_{2}(1.0) / \mathrm{TEMPO}(2.0)\end{array}\right.}\end{array}$ & $\left(\mathrm{CF}_{3}\right)_{2} \mathrm{CHOH} / 70^{\circ} \mathrm{C} / 11 \mathrm{~h}$ & 69 \\
\hline 13 & $\begin{array}{l}\text { sulphonium salt (1.6)/CuBr} 2(0.3) \\
{\left[\mathrm{Ru}(\mathrm{p} \text {-cymene }) \mathrm{Cl}_{2}\right]_{2}(0.2) / \mathrm{L} 2(0.25) / \mathrm{KF}(2.0)} \\
\mathrm{MgCl}_{2}(1.0) / \mathrm{TEMPO}(3.0)\end{array}$ & $\left(\mathrm{CF}_{3}\right)_{2} \mathrm{CHOH} / 70{ }^{\circ} \mathrm{C} / 11 \mathrm{~h}$ & 55 \\
\hline 14 & $\begin{array}{c}\text { sulphonium salt (1.6)/CuBr} 2(0.3) \\
{\left[\mathrm{Ru}(\mathrm{p} \text {-cymene }) \mathrm{Cl}_{2}\right]_{2}(0.2) / \mathrm{L} 2(0.25) / \mathrm{KF}(2.0)} \\
\mathrm{MgCl}_{2}(1.0) \text {-in dark }\end{array}$ & $\left(\mathrm{CF}_{3}\right)_{2} \mathrm{CHOH} / 70^{\circ} \mathrm{C} / 11 \mathrm{~h}$ & 86 \\
\hline
\end{tabular}

${ }^{\mathrm{a}}$ Isolated yield. ${ }^{\mathrm{b}}$ All reactions were conducted at room temperature in inert atmosphere. 

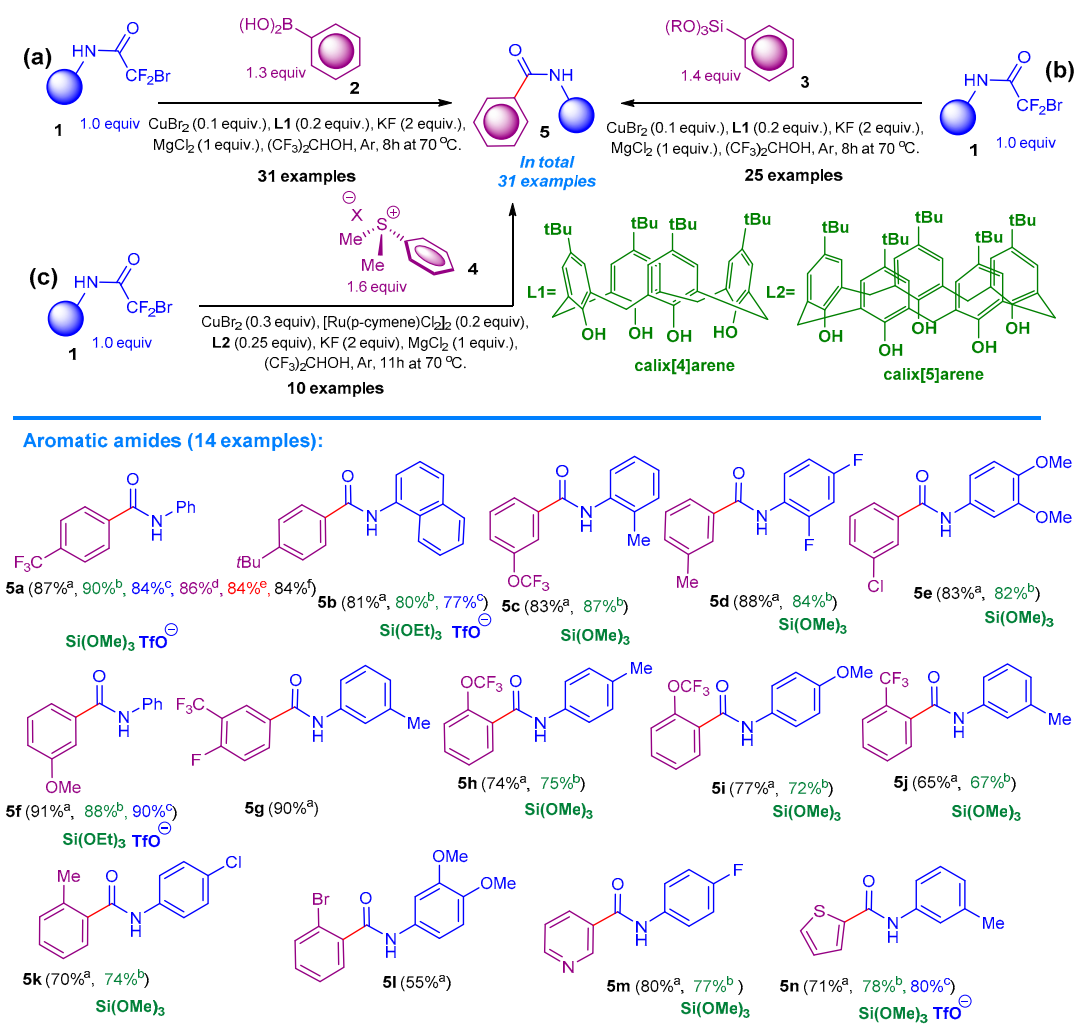

aUsing boronic acids (14 examples); bUsing aryl trialcoxysilanes (12 examples); ' Using dimethyl(aryl)sulfonium salts (4 example); 'Using potassium aryltrifluoroborate (1 example); ${ }^{\circledR} U s i n g$ aryl pinacol boronates ( 1 example); ${ }^{f} U s i n g$ boronic acid and 2,2-difluoro-2-iodoacetamide (1 example).
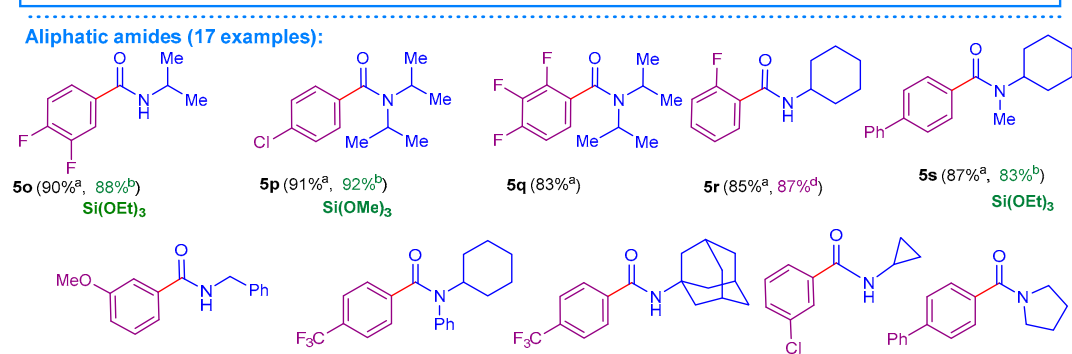

$\mathbf{5 t}\left(90 \%^{\mathrm{a}}, 87 \%^{\mathrm{b}}, 84 \%^{\mathrm{c}}, 80 \%^{\mathrm{d}}, 83 \%^{\mathrm{e}}, 87 \%^{\mathrm{f}}\right) \mathbf{5 u}\left(90 \%^{\mathrm{a}}, 90 \%^{\mathrm{b}}, 81 \%^{\mathrm{c}}\right) \quad \mathbf{5 v}\left(87 \%^{\mathrm{a}}, 90 \%^{\mathrm{b}}, 84 \%^{\mathrm{d}}\right) \mathbf{5} \mathbf{w}\left(65 \%^{\mathrm{a}}, 64 \%^{\mathrm{b}}, 58 \%\right) \mathbf{5 x}\left(91 \%^{\mathrm{a}}, 89 \%^{\mathrm{b}}, 82 \%^{\mathrm{c}}\right)$

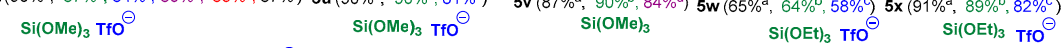<smiles>O=C(c1ccc(F)cc1)c1ccc(CNC(=O)c2ccccc2C(=O)Nc2c(Cl)cccc2C(=O)NCc2ccccc2)cc1</smiles>

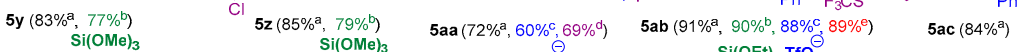

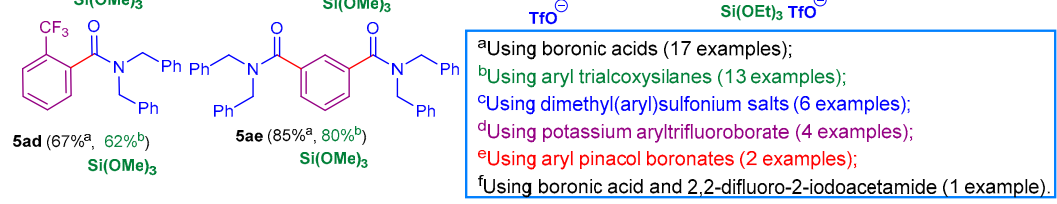
Scale-up synthesis (4 examples):

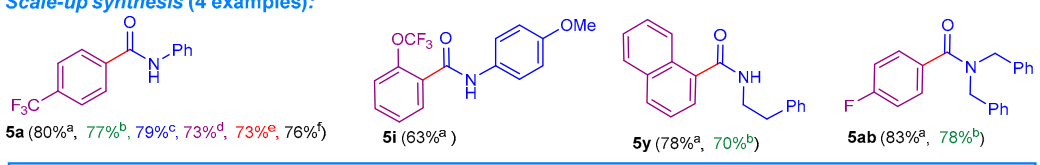

aUsing boronic acids (4 examples); bUsing aryl trialcoxysilanes ( 3 examples); 'Using dimethyl(aryl)sulfonium salts (1 example); dUsing potassium aryltrifluoroborate (1 example); eUsing aryl pinacol boronates (1 example); fUsing boronic acid and 2,2-difluoro-2-iodoacetamide (1 example).

Scheme 3. (a) Reactions of 2-bromo-2,2-difluoro-acetamides with aryl boronic acid, (b) Reactions of 2bromo-2,2-difluoro-acetamides with aryl trialkoxysilanes, (c) Reactions of 2-bromo-2,2-difluoro-acetamides with dimethyl-aryl-sulfonium triflates. Product scope of amides using developed synthetic protocols. 
To the general scope and limitations, it is also important to note: (1) Within both described syntactic protocols we tried numerous other N-substituted and N-unsubstituted derivatives of 2-bromo-2,2-difluoroacetic acid, for instance: 2-bromo-2,2-difluoroethanethioamides, 2-bromo-2,2-difluoroacetimidamides, 2-bromo-2,2-difluoroacetohydrazonamides; all these substrates were not prone to enter the developed arylation protocols; (2) Aryl pinacol borates as well as aryl trifluoroborates in the form of potassium salts act as arylation agents in the frames of both synthetic protocols ( 2 and 4 examples respectively, Scheme 3); (3) 2,2-Difluoro-2-iodoacetamides exerted similar activity as the corresponding bromo derivatives (2 examples, Scheme 3).

As the final accord of this work, we turned our attention to aryl sulphonium salts 4 . These are donors of aryl groups and are often considered as equivalents of aryl halides, possessing low reduction potentials [46-48]. We assumed that those species might have capacity to enter the title synthetic protocol (Scheme 2c). These compounds did not react well under previously optimized reaction conditions, where the model compound 5a was obtained in 47\% yield (Table 3, Entry 1). Thus, we embarked once more on the search for new operational reaction conditions for the model reaction. It is worthwhile to note that in the case of this reaction, we had to increase the amount of copper salt to 0.3 equiv. and add 0.2 equiv. of $\left[\mathrm{Ru}\left(\mathrm{p} \text {-cymene) } \mathrm{Cl}_{2}\right]_{2}\right.$, which was superior to other TM co-catalysts (Table 3). Finally, by employing $\mathrm{CuBr}_{2}$ (0.3 equiv.), [ $\mathrm{Ru}\left(\mathrm{p} \text {-cymene) } \mathrm{Cl}_{2}\right]_{2}$ (0.2 equiv.), $\mathrm{KF}$ (2 equiv.), $\mathrm{MgCl}_{2}$ (1 equiv.) and 0.25 equiv. of calix[5]arene derivative (L2), in hexafluoropropanol, the model amide 5a was prepared in $84 \%$ yield. Further study of the scope resulted in the preparation of ten amides in total (Scheme 3c).

To gain the insight to the reaction mechanism, we performed several control experiments: (a) Reactions without addition of calixarenes; (b) reactions without $\mathrm{CuBr}_{2}$ and $\mathrm{MgCl}_{2}$; (c) reactions in the dark and (d) reactions with 2 equiv. and 3 equiv. of TEMPO, which led to the modest decrease of the yield of title model amid compound. All these experiments are depicted in the Tables 1-3.

\section{Materials and Methods}

Commercially available starting materials, reagents, catalysts, anhydrous, and degassed solvents were used without further purification. Flash column chromatography was performed with Merck Silica gel 60 (230-400 mesh). The solvents for column chromatography were distilled before the use. Thin layer chromatography was carried out using Merck TLC Silica gel 60 F254 and visualized by short-wavelength ultraviolet light or by treatment with potassium permanganate $\left(\mathrm{KMnO}_{4}\right)$ stain. ${ }^{1} \mathrm{H},{ }^{13} \mathrm{C}$, and ${ }^{19} \mathrm{~F}-\mathrm{NMR}$ spectra were recorded on a Bruker 250 and $500 \mathrm{MHz}$ at $20{ }^{\circ} \mathrm{C}$. All ${ }^{1} \mathrm{H}-\mathrm{NMR}$ spectra are reported in parts per million ( $\mathrm{ppm}$ ) downfield of TMS and were measured relative to the signals for $\mathrm{CHCl} 3$ (7.26 ppm) and DMSO (2.50 ppm). All ${ }^{13} \mathrm{C}\left\{{ }^{1} \mathrm{H}\right\}-\mathrm{NMR}$ spectra were reported in ppm relative to residual $\mathrm{CHCl}_{3}(77.00 \mathrm{ppm})$ or DMSO $(39.70 \mathrm{ppm})$ and were obtained with ${ }^{1} \mathrm{H}$ decoupling. Coupling constants, J, are reported in Hertz $(\mathrm{Hz})$. Gas chromatographic analyses was performed on Gas Chromatograph Mass Spectrometer GCMS-QP2010 Ultra instrument.

The optimal reaction conditions were identified by microscale high-throughput experimentation screening. Parallel synthesis was accomplished in an MBraun glovebox operating with a constant Ar-purge (oxygen and water $<5 \mathrm{ppm}$ ). Screening reactions were carried out in $10 \mathrm{~mL}$ vials using suitable heating blocks. Liquid chemicals were dosed using gas tight micro syringes. Isolation of obtained compounds was achieved by column chromatography on Silica gel.

All used boronic acids 2 and some aryl trialkoxysilanes $\mathbf{3}$ are commercially available and were purchased from appropriate vendors. 2-Bromo-2,2-difluoroacetamides [49-57], 1, 2-iodo-2,2-difluoroacetamides [54], aryl trialkoxysilanes 3 [58-64], sulfonium salts 4 [65-67], and calixarenes L1, L2 $[68,69]$ are known compounds in the literature and were prepared according to the known literature, and the spectral data are identical with the corresponding literature. Copies ${ }^{1} \mathrm{H}$ and ${ }^{13} \mathrm{C}$-NMR spectra are placed in Supplementary Materials. 
General procedure for the synthesis of amides 5 by the reaction of 2-bromo-2,2-difluoroacetamides 1 with aryl boronic acids 2.

Under inert atmosphere (glovebox operating with a constant Ar-purge), to an $18 \mathrm{~mL}$ ACE pressure tube equipped with a stir bar, consequently, an appropriate 2-bromo-2,2difluoroacetamide (1.0 mmol, 1.0 equiv.), $\mathrm{KF}$ (116 mg, $2.0 \mathrm{mmol}, 2.0$ equiv.), $\mathrm{MgCl}_{2}$ (95 mg, $1.0 \mathrm{mmol}, 1.0$ equiv.), appropriate aryl boronic acid ( $1.3 \mathrm{mmol}, 1.3$ equiv.), the $\mathbf{L 1}(0.2 \mathrm{mmol}$, 0.2 equiv.), and finally $\mathrm{CuBr}_{2}(0.1 \mathrm{mmol}, 0.1$ equiv. $)$ were placed; then the hexafluoropropanol $(0.12 \mathrm{mmol} / \mathrm{mL})$ was added and the reaction vessel was properly capped by Teflon stopper. Finally, the reaction vessel was removed from the glovebox and subjected to heating under vigorous stirring for $8 \mathrm{~h}$. The progress of the reaction was controlled by TLC. After completion, the reaction mixture was evaporated until it reached dryness using a rotary evaporator, the content of the flask was generously treated with distilled water, filtered, and finally properly dried in vacuum. The resulting crude was directly subjected to gradient flash chromatography on silica gel using a mixture of hexane/ethyl acetate as eluent to isolate the desired amide derivative.

The gram scale synthesis was performed on $10 \mathrm{mmol}$ of the starting 2-bromo-2,2difluoroacetamide.

General procedure for the synthesis of amides 5 by the reaction of 2-bromo-2,2-difluoroacetamides 1 with aryl trialkoxysilanes 3.

Under inert atmosphere (glovebox operating with a constant Ar-purge), to an $18 \mathrm{~mL}$ ACE pressure tube equipped with a stir bar, an appropriate 2-bromo-2,2-difluoroacetamide (1.0 mmol, 1.0 equiv.), $\mathrm{KF}$ (116 mg, $2.0 \mathrm{mmol}, 2.0$ equiv.), $\mathrm{MgCl}_{2}$ (95 mg, $1.0 \mathrm{mmol}, 1.0$ equiv.), appropriate trialkoxysilane (1.4 mmol, 1.4 equiv.), the L1 ( $0.2 \mathrm{mmol}, 0.2$ equiv.), and finally $\mathrm{CuBr}_{2}$ ( $0.1 \mathrm{mmol}, 0.1$ equiv.) was consequently placed; then the hexafluoropropanol $(0.12 \mathrm{mmol} / \mathrm{mL})$ was added and the reaction vessel was properly capped by Teflon stopper. Finally, the reaction vessel was removed from the glovebox and subjected to heating under vigorous stirring for $8 \mathrm{~h}$. The progress of the reaction was controlled by TLC. After completion, the reaction mixture was evaporated until it reached dryness using a rotary evaporator, the content of the flask was generously treated with distilled water, filtered, and finally properly dried in vacuum. The resulting crude was directly subjected to gradient flash chromatography on silica gel using a mixture of hexane/ethyl acetate as eluent to isolate the desired amide derivative.

The gram scale synthesis was performed on $10 \mathrm{mmol}$ of the starting 2-bromo-2,2difluoroacetamide.

General procedure for the synthesis of amides 5 by the reaction of 2-bromo-2,2-difluoroacetamides 1 with (aryl)dimethylsulfonium salts 4.

Under inert atmosphere (glovebox operating with a constant Ar-purge), to an $18 \mathrm{~mL}$ ACE pressure equipped with a stir bar, an appropriate 2-bromo-2,2-difluoroacetamide (1.0 mmol, 1.0 equiv.), KF (116 mg, $2.0 \mathrm{mmol}, 2.0$ equiv.), $\mathrm{MgCl}_{2}$ (95 mg, $1.0 \mathrm{mmol}$, 1.0 equiv.), aryl sulphonium salt (1.6 mmol, 1.6 equiv.), the L2 ( $0.25 \mathrm{mmol}, 0.25$ equiv.), $\left[\mathrm{Ru} \text { (p-cymene) } \mathrm{Cl}_{2}\right]_{2}(0.2 \mathrm{mmol}, 0.2$ equiv. $)$, and finally $\mathrm{CuBr}_{2}(0.3 \mathrm{mmol}, 0.3$ equiv. $)$ was consequently placed; then the hexafluoropropanol $(0.12 \mathrm{mmol} / \mathrm{mL})$ was added and the reaction vessel was properly capped by Teflon stopper. Finally, the reaction vessel was removed from the glovebox and subjected to heating under vigorous stirring for $11 \mathrm{~h}$. The progress of the reaction was controlled by TLC. After completion, the reaction mixture was evaporated until it reached dryness using rotary evaporator, the content of the flask was generously treated with distilled water, filtered, and finally properly dried in vacuum. The resulting crude was directly subjected to gradient flash chromatography on silica gel using a mixture of hexane/ethyl acetate as eluent to isolate the desired amide derivative. The gram scale synthesis was performed on $10 \mathrm{mmol}$ of the starting 2-bromo-2,2-difluoroacetamide.

N-phenyl-4-(trifluoromethyl)benzamide 5a. The title compound was prepared, starting with 2-bromo-2,2-difluoroacetamide $1 \mathrm{a}(250 \mathrm{mg}, 1.0 \mathrm{mmol}, 1.0$ equiv.), KF (116 mg, $2.0 \mathrm{mmol}, 2.0$ equiv.), $\mathrm{MgCl}_{2}$ (95 mg, $1.0 \mathrm{mmol}, 1.0$ equiv.), aryl boronic acid $2 \mathrm{n}$ ( $247 \mathrm{mg}$, $1.3 \mathrm{mmol}, 1.3$ equiv.), L1 (130 mg, $0.2 \mathrm{mmol}, 0.2$ equiv.), $\mathrm{CuBr}_{2}$ (22.3 mg, $0.1 \mathrm{mmol}, 0.1$ equiv.), 
and hexafluoropropanol $(0.12 \mathrm{mmol} / \mathrm{mL})$. The purification of the dry crude performed by column chromatography on silica gel provides the amide 5 a $(231 \mathrm{mg}, 0.87 \mathrm{mmol}$, $87 \%$ ). The gram scale synthesis was performed on $10 \mathrm{mmol}$ of the starting 2-bromo2,2-difluoroacetamide $1 \mathbf{a}$ and the amide $5 \mathbf{a}$ was prepared in $80 \%$ yield $(2.12 \mathrm{~g}, 8 \mathrm{mmol})$. Alternatively, the title compound was prepared starting with an appropriate 2-bromo-2,2difluoroacetamide 1a (250 mg, $1.0 \mathrm{mmol}, 1.0$ equiv.), KF (116 mg, $2.0 \mathrm{mmol}, 2.0$ equiv.), $\mathrm{MgCl}_{2}$ (95 mg, $1.0 \mathrm{mmol}, 1.0$ equiv.), appropriate trialkoxysilane $3 \mathrm{k}$ (372 mg, $1.4 \mathrm{mmol}$, 1.4 equiv.), the $\mathbf{L} 1$ ( $130 \mathrm{mg}, 0.2 \mathrm{mmol}, 0.2$ equiv.), $\mathrm{CuBr}_{2}$ ( $22.3 \mathrm{mg}, 0.1 \mathrm{mmol}, 0.1$ equiv.), and hexafluoropropanol $(0.12 \mathrm{mmol} / \mathrm{mL})$. The purification of the dry crude performed by column chromatography on silica gel provides the amide 5 a $(239 \mathrm{mg}, 0.90 \mathrm{mmol}$, $90 \%$ ). The gram scale synthesis was performed on $10 \mathrm{mmol}$ of the starting 2-bromo-2,2difluoroacetamide $1 \mathbf{a}$ and the amide $5 \mathbf{a}$ was prepared in $77 \%$ yield $(2.04 \mathrm{~g}, 7.7 \mathrm{mmol})$.

Alternatively, the title compound was prepared starting with an appropriate 2-bromo2,2-difluoroacetamide 1a (250 mg, $1.0 \mathrm{mmol}, 1.0$ equiv.), $\mathrm{KF}$ (116 mg, $2.0 \mathrm{mmol}, 2.0$ equiv.), $\mathrm{MgCl}_{2}$ (95 mg, $1.0 \mathrm{mmol}, 1.0$ equiv.), aryl sulphonium salt $4 \mathbf{a}(584 \mathrm{mg}, 1.6 \mathrm{mmol}, 1.6$ equiv.), the $\mathbf{L} 2$ (202 mg, $0.25 \mathrm{mmol}, 0.25$ equiv.), [ $\mathrm{Ru}\left(\mathrm{p} \text {-cymene) } \mathrm{Cl}_{2}\right]_{2}$ (122 mg, $0.2 \mathrm{mmol}, 0.2$ equiv.), $\mathrm{CuBr}_{2}$ (67 mg, $0.3 \mathrm{mmol}, 0.3$ equiv.), and hexafluoropropanol $(0.12 \mathrm{mmol} / \mathrm{mL})$. The purification of the dry crude performed by column chromatography on silica gel provides the amide $5 \mathbf{a}(222 \mathrm{mg}, 0.84 \mathrm{mmol}, 84 \%)$. The gram scale synthesis was performed on $10 \mathrm{mmol}$ of the starting 2-bromo-2,2-difluoroacetamide $1 \mathbf{a}$ and the amide $5 \mathbf{a}$ was prepared in $79 \%$ yield $(2.09 \mathrm{~g}, 7.9 \mathrm{mmol})$.

Alternatively, the title compound was prepared starting with an appropriate 2-bromo2,2-difluoroacetamide $1 \mathrm{a}$ (250 mg, $1.0 \mathrm{mmol}, 1.0$ equiv.), KF (116 mg, $2.0 \mathrm{mmol}, 2.0$ equiv.), $\mathrm{MgCl}_{2}$ (95 mg, $1.0 \mathrm{mmol}, 1.0$ equiv.), appropriate potassium trifluoro(4-(trifluoromethyl) phenyl)borate ( $328 \mathrm{mg}, 1.3 \mathrm{mmol}, 1.3$ equiv.), the $\mathbf{L 1}$ ( $130 \mathrm{mg}, 0.2 \mathrm{mmol}, 0.2$ equiv.), $\mathrm{CuBr}_{2}$ (22.3 $\mathrm{mg}, 0.1 \mathrm{mmol}, 0.1$ equiv.), and hexafluoropropanol $(0.12 \mathrm{mmol} / \mathrm{mL})$. The purification of the dry crude performed by column chromatography on silica gel provides the amide $5 a(228 \mathrm{mg}, 0.86 \mathrm{mmol}, 86 \%)$. The gram scale synthesis was performed on $10 \mathrm{mmol}$ of the starting 2-bromo-2,2-difluoroacetamide $1 \mathbf{a}$ and the amide $5 \mathbf{a}$ was prepared in $73 \%$ yield $(1.93 \mathrm{~g}, 7.3 \mathrm{mmol})$.

Alternatively, the title compound was prepared starting with an appropriate 2-bromo2,2-difluoroacetamide $1 \mathrm{a}(250 \mathrm{mg}, 1.0 \mathrm{mmol}, 1.0$ equiv.), $\mathrm{KF}(116 \mathrm{mg}, 2.0 \mathrm{mmol}, 2.0$ equiv.), $\mathrm{MgCl}_{2}$ (95 mg, $1.0 \mathrm{mmol}, 1.0$ equiv.), appropriate 4,4,5,5-tetramethyl-2-(4-(trifluoromethyl) phenyl)-1,3,2-dioxaborolane (354 mg, $1.3 \mathrm{mmol}, 1.3$ equiv.), the $\mathbf{L 1}(130 \mathrm{mg}, 0.2 \mathrm{mmol}$, 0.2 equiv.), $\mathrm{CuBr}_{2}(22.3 \mathrm{mg}, 0.1 \mathrm{mmol}, 0.1$ equiv.), and hexafluoropropanol $(0.12 \mathrm{mmol} / \mathrm{mL})$. The purification of the dry crude performed by column chromatography on silica gel provides the amide $5 \mathbf{a}(222 \mathrm{mg}, 0.84 \mathrm{mmol}, 84 \%)$. The gram scale synthesis was performed on $10 \mathbf{m m o l}$ of the starting 2-bromo-2,2-difluoroacetamide $\mathbf{1 a}$ and the amide $\mathbf{5 a}$ was prepared in $73 \%$ yield $(1.93 \mathrm{~g}, 7.3 \mathrm{mmol})$.

Alternatively, the title compound was prepared starting with an appropriate 2,2difluoro-2-iodo-N-phenylacetamide ( $297 \mathrm{mg}, 1.0 \mathrm{mmol}, 1.0$ equiv.), KF (116 mg, $2.0 \mathrm{mmol}$, 2.0 equiv.), $\mathrm{MgCl}_{2}$ (95 mg, $1.0 \mathrm{mmol}, 1.0$ equiv.), appropriate aryl boronic acid $\mathbf{2 n}$ ( $247 \mathrm{mg}$, $1.3 \mathrm{mmol}, 1.3$ equiv.), the $\mathbf{L} 1$ ( $130 \mathrm{mg}, 0.2 \mathrm{mmol}, 0.2$ equiv.), $\mathrm{CuBr}_{2}(22.3 \mathrm{mg}, 0.1 \mathrm{mmol}$, 0.1 equiv.), and hexafluoropropanol $(0.12 \mathrm{mmol} / \mathrm{mL})$. The purification of the dry crude performed by column chromatography on silica gel provides the amide $5 \mathrm{a}(222 \mathrm{mg}, 0.84 \mathrm{mmol}$, $84 \%$ ). The gram scale synthesis was performed on $10 \mathrm{mmol}$ of the starting 2-bromo-2,2difluoroacetamide $1 \mathbf{a}$ and the amide $5 \mathbf{a}$ was prepared in $76 \%$ yield $(2.01 \mathrm{~g}, 7.6 \mathrm{mmol})$.

Flash column chromatography was performed using a mixture of hexane/ethyl acetate 3:1 as an eluent to provide the corresponding amide product.

White solid, mp 184-185 ${ }^{\circ} \mathrm{C} .{ }^{1} \mathrm{H}-\mathrm{NMR}\left(500 \mathrm{MHz}, \mathrm{DMSO}-d_{6}\right): \delta 7.12\left(\mathrm{t}, 1 \mathrm{H},{ }^{3} \mathrm{~J}=7.3 \mathrm{~Hz}\right.$, $\left.\mathrm{CH}_{\mathrm{Ar}}\right), 7.37\left(\mathrm{t}, 2 \mathrm{H},{ }^{3} \mathrm{~J}=8.3 \mathrm{~Hz}, \mathrm{CH}_{\mathrm{Ar}}\right), 7.80\left(\mathrm{~d}, 2 \mathrm{H},{ }^{3} \mathrm{~J}=7.6 \mathrm{~Hz}, \mathrm{CH}_{\mathrm{Ar}}\right), 7.89\left(\mathrm{~d}, 2 \mathrm{H},{ }^{3} \mathrm{~J}=8.2 \mathrm{~Hz}\right.$, $\left.\mathrm{CH}_{\mathrm{Ar}}\right), 8.16\left(\mathrm{~d}, 2 \mathrm{H},{ }^{3} \mathrm{~J}=8.1 \mathrm{~Hz}, \mathrm{CH}_{\mathrm{Ar}}\right), 10.5(\mathrm{~s}, 1 \mathrm{H}, \mathrm{NH})$.

${ }^{13} \mathrm{C}\left\{{ }^{1} \mathrm{H}\right\}$-NMR $\left(126 \mathrm{MHz}, \mathrm{DMSO}-d_{6}\right): \delta 120.5,123.9\left(\mathrm{q},{ }^{1} J_{C F}=273.8 \mathrm{~Hz}, \mathrm{CF}_{3}\right), 124.0,125.4$ $\left(\mathrm{d}, J_{C F}=3.1 \mathrm{~Hz}\right), 128.6,128.7,131.4\left(\mathrm{q},{ }^{2} J_{C F}=30.3 \mathrm{~Hz}, C_{C F}\right), 138.8\left(\mathrm{~d}, J_{C F}=11.3 \mathrm{~Hz}\right), 164.4$. 
HRMS (TOF MS ES+): Calcd for $\mathrm{C}_{14} \mathrm{H}_{11} \mathrm{NOF}_{3}(\mathrm{M}+\mathrm{H})$ 266.0809. Found 266.0793.

4-(tert-butyl)-N-(naphthalen-1-yl)benzamide $5 \mathrm{~b}$. The title compound was prepared starting with an appropriate 2-bromo-2,2-difluoroacetamide 1 e $(300 \mathrm{mg}, 1.0 \mathrm{mmol}$, 1.0 equiv.), $\mathrm{KF}$ (116 mg, $2.0 \mathrm{mmol}, 2.0$ equiv.), $\mathrm{MgCl}_{2}$ ( $95 \mathrm{mg}, 1.0 \mathrm{mmol}, 1.0$ equiv.), appropriate aryl boronic acid $\mathbf{2 a}(231 \mathrm{mg}, 1.3 \mathrm{mmol}, 1.3$ equiv. $)$, the $\mathbf{L 1}(130 \mathrm{mg}, 0.2 \mathrm{mmol}$, 0.2 equiv.), $\mathrm{CuBr}_{2}(22.3 \mathrm{mg}, 0.1 \mathrm{mmol}, 0.1$ equiv.), and hexafluoropropanol $(0.12 \mathrm{mmol} / \mathrm{mL})$. The purification of the dry crude performed by column chromatography on silica gel provides the amide $5 \mathrm{~b}(245 \mathrm{mg}, 0.81 \mathrm{mmol}, 81 \%)$.

Alternatively, the title compound was prepared starting with an appropriate 2-bromo2,2-difluoroacetamide 1 e (300 mg, $1.0 \mathrm{mmol}, 1.0$ equiv.), $\mathrm{KF}(116 \mathrm{mg}, 2.0 \mathrm{mmol}, 2.0$ equiv.), $\mathrm{MgCl}_{2}$ (95 mg, $1.0 \mathrm{mmol}, 1.0$ equiv.), appropriate trialkoxysilane $3 \mathrm{a}(414 \mathrm{mg}, 1.4 \mathrm{mmol}$, 1.4 equiv.), the $\mathbf{L} 1$ ( $130 \mathrm{mg}, 0.2 \mathrm{mmol}, 0.2$ equiv.), $\mathrm{CuBr}_{2}$ ( $22.3 \mathrm{mg}, 0.1 \mathrm{mmol}, 0.1$ equiv.), and hexafluoropropanol $(0.12 \mathrm{mmol} / \mathrm{mL})$. The purification of the dry crude performed by column chromatography on silica gel provides the amide $\mathbf{5 b}(242 \mathrm{mg}, 0.80 \mathrm{mmol}, 80 \%)$.

Alternatively, the title compound was prepared starting with an appropriate 2-bromo2,2-difluoroacetamide 1e (300 mg, $1.0 \mathrm{mmol}, 1.0$ equiv.), $\mathrm{KF}(116 \mathrm{mg}, 2.0 \mathrm{mmol}, 2.0$ equiv.), $\mathrm{MgCl}_{2}$ (95 mg, $1.0 \mathrm{mmol}, 1.0$ equiv.), aryl sulphonium salt $4 \mathrm{~h}$ (312 mg, $1.6 \mathrm{mmol}, 1.6$ equiv.), the $\mathbf{L} 2$ (202 mg, $0.25 \mathrm{mmol}, 0.25$ equiv.), [ $\mathrm{Ru}\left(\mathrm{p} \text {-cymene) } \mathrm{Cl}_{2}\right]_{2}$ (122 $\mathrm{mg}, 0.2 \mathrm{mmol}, 0.2$ equiv.), $\mathrm{CuBr}_{2}(67 \mathrm{mg}, 0.3 \mathrm{mmol}, 0.3$ equiv.), and hexafluoropropanol $(0.12 \mathrm{mmol} / \mathrm{mL})$. The purification of the dry crude performed by column chromatography on silica gel provides the amide $5 \mathbf{b}(233 \mathrm{mg}, 0.77 \mathrm{mmol}, 77 \%)$.

Flash column chromatography was performed using a mixture of hexane/ethyl acetate 3:1 as an eluent to provide the corresponding amide product.

White solid, mp 146-147 ${ }^{\circ} \mathrm{C} .{ }^{1} \mathrm{H}-\mathrm{NMR}\left(500 \mathrm{MHz}, \mathrm{CDCl}_{3}\right)$ : $\delta 1.38(\mathrm{~s}, 9 \mathrm{H}, t \mathrm{Bu}), 7.46-7.51$ $\left(\mathrm{m}, 5 \mathrm{H}, \mathrm{CH}_{\mathrm{Ar}}\right), 7.72\left(\mathrm{~d}, 1 \mathrm{H},{ }^{3} \mathrm{~J}=8.4 \mathrm{~Hz}, \mathrm{CH}_{\mathrm{Ar}}\right), 7.87-7.90\left(\mathrm{~m}, 3 \mathrm{H}, \mathrm{CH}_{\mathrm{Ar}}\right), 7.92\left(\mathrm{~s}, 1 \mathrm{H}, \mathrm{CH}_{\mathrm{Ar}}\right)$, 7.97 (br. s, $\left.1 \mathrm{H}, \mathrm{CH}_{\mathrm{Ar}}\right), 8.33(\mathrm{~s}, 1 \mathrm{H}, \mathrm{NH})$.

$\left.{ }^{13} \mathrm{C}_{1}{ }^{1} \mathrm{H}\right\}$-NMR $\left(126 \mathrm{MHz}, \mathrm{CDCl}_{3}\right): \delta 31.2,35.0,120.79,120.80,121.2,125.7,125.9,126.3$, 127.1, 127.5, 127.51, 128.7, 131.9, 132.5, 134.1.

HRMS (TOF MS ES+): Calcd for $\mathrm{C}_{21} \mathrm{H}_{22} \mathrm{NO}(\mathrm{M}+\mathrm{H})$ 304.1707. Found 304.1701.

$\mathrm{N}$-(o-tolyl)-3-(trifluoromethoxy)benzamide $5 \mathrm{c}$. The title compound was prepared starting with an appropriate 2-bromo-2,2-difluoroacetamide 1d $(264 \mathrm{mg}, 1.0 \mathrm{mmol}$, 1.0 equiv.), KF (116 mg, $2.0 \mathrm{mmol}, 2.0$ equiv.), $\mathrm{MgCl}_{2}$ (95 mg, $1.0 \mathrm{mmol}, 1.0$ equiv.), appropriate aryl boronic acid $2 \mathbf{r}(268 \mathrm{mg}, 1.3 \mathrm{mmol}, 1.3$ equiv.), the $\mathbf{L 1}(130 \mathrm{mg}, 0.2 \mathrm{mmol}$, 0.2 equiv.), $\mathrm{CuBr}_{2}$ (22.3 mg, $0.1 \mathrm{mmol}, 0.1$ equiv.), and hexafluoropropanol $(0.12 \mathrm{mmol} / \mathrm{mL})$. The purification of the dry crude performed by column chromatography on silica gel provides the amide $5 \mathrm{c}(245 \mathrm{mg}, 0.83 \mathrm{mmol}, 83 \%)$.

Alternatively, the title compound was prepared starting with an appropriate 2-bromo2,2-difluoroacetamide $1 \mathbf{d}$ ( $264 \mathrm{mg}, 1.0 \mathrm{mmol}, 1.0$ equiv.), $\mathrm{KF}$ (116 mg, $2.0 \mathrm{mmol}, 2.0$ equiv.), $\mathrm{MgCl}_{2}$ (95 mg, $1.0 \mathrm{mmol}$, 1.0 equiv.), appropriate trialkoxysilane $3 \mathbf{n}(395 \mathrm{mg}, 1.4 \mathrm{mmol}$, 1.4 equiv.), the $\mathbf{L} 1$ (130 mg, $0.2 \mathrm{mmol}, 0.2$ equiv.) $\mathrm{CuBr}_{2}$ (22.3 mg, $0.1 \mathrm{mmol}, 0.1$ equiv.), and hexafluoropropanol $(0.12 \mathrm{mmol} / \mathrm{mL})$. The purification of the dry crude performed by column chromatography on silica gel provides the amide $5 \mathrm{c}(257 \mathrm{mg}, 0.87 \mathrm{mmol}, 87 \%)$.

Flash column chromatography was performed using a mixture of hexane/ethyl acetate 5:1 as an eluent to provide the corresponding amide product.

White solid, mp 94-95 ${ }^{\circ} \mathrm{C} .{ }^{1} \mathrm{H}-\mathrm{NMR}\left(500 \mathrm{MHz}, \mathrm{CDCl}_{3}\right): \delta 2.30(\mathrm{~s}, 3 \mathrm{H}, \mathrm{Me}), 7.12-7.15$ $\left(\mathrm{m}, 1 \mathrm{H}, \mathrm{CH}_{\mathrm{Ar}}\right), 7.21-7.24\left(\mathrm{~m}, 2 \mathrm{H}, \mathrm{CH}_{\mathrm{Ar}}\right), 7.40\left(\mathrm{~d}, 1 \mathrm{H},{ }^{3} \mathrm{~J}=8.1 \mathrm{~Hz}, \mathrm{CH}_{\mathrm{Ar}}\right), 7.50(\mathrm{t}, 1 \mathrm{H}$, $\left.{ }^{3} J=7.9 \mathrm{~Hz}, \mathrm{CH}_{\mathrm{Ar}}\right), 7.74-7.78\left(\mathrm{~m}, 3 \mathrm{H}, \mathrm{CH}_{\mathrm{Ar}}\right), 7.81(\mathrm{br} \mathrm{s}, 1 \mathrm{H}, \mathrm{NH})$.

$\left.{ }^{13} \mathrm{C}_{1}{ }^{1} \mathrm{H}\right\}$-NMR $\left(126 \mathrm{MHz}, \mathrm{CDCl}_{3}\right): \delta 17.8,120.1,120.4\left(\mathrm{q},{ }^{1} J_{\mathrm{CF}}=258.6 \mathrm{~Hz}\right), 123.6,124.1$, $125.1,125.9,126.8,130.0,130.2,130.6,135.2,137.0,149.5,164.2$.

HRMS (TOF MS ES+): Calcd for $\mathrm{C}_{15} \mathrm{H}_{13} \mathrm{NO}_{2} \mathrm{~F}_{3}(\mathrm{M}+\mathrm{H})$ 296.0901. Found 296.0898.

$\mathrm{N}$-(2,4-difluorophenyl)-3-methylbenzamide $5 \mathrm{~d}$. The title compound was prepared starting with an appropriate 2-bromo-2,2-difluoroacetamide $\mathbf{1 j}(286 \mathrm{mg}, 1.0 \mathrm{mmol}$, 1.0 equiv.), $\mathrm{KF}$ (116 mg, $2.0 \mathrm{mmol}, 2.0$ equiv.), $\mathrm{MgCl}_{2}$ (95 mg, $1.0 \mathrm{mmol}, 1.0$ equiv.), appropriate aryl boronic acid $\mathbf{2 b}(177 \mathrm{mg}, 1.3 \mathrm{mmol}, 1.3$ equiv.), the $\mathbf{L 1}(130 \mathrm{mg}, 0.2 \mathrm{mmol}$, 
0.2 equiv.), $\mathrm{CuBr}_{2}$ ( $22.3 \mathrm{mg}, 0.1 \mathrm{mmol}, 0.1$ equiv.), and hexafluoropropanol $(0.12 \mathrm{mmol} / \mathrm{mL})$. The purification of the dry crude performed by column chromatography on silica gel provides the amide $5 \mathrm{~d}(217 \mathrm{mg}, 0.88 \mathrm{mmol}, 88 \%)$.

Alternatively, the title compound was prepared starting with an appropriate 2-bromo2,2-difluoroacetamide 1 j ( $286 \mathrm{mg}$, $1.0 \mathrm{mmol}, 1.0$ equiv.), KF (116 mg, $2.0 \mathrm{mmol}, 2.0$ equiv.), $\mathrm{MgCl}_{2}$ (95 mg, $1.0 \mathrm{mmol}$, 1.0 equiv.), appropriate trialkoxysilane $3 \mathbf{b}(297 \mathrm{mg}, 1.4 \mathrm{mmol}$, 1.4 equiv.), the $\mathbf{L} 1$ (130 mg, $0.2 \mathrm{mmol}, 0.2$ equiv.), $\mathrm{CuBr}_{2}$ ( $22.3 \mathrm{mg}, 0.1 \mathrm{mmol}, 0.1$ equiv.), and hexafluoropropanol $(0.12 \mathrm{mmol} / \mathrm{mL})$. The purification of the dry crude performed by column chromatography on silica gel provides the amide $5 \mathrm{~d}(207 \mathrm{mg}, 0.84 \mathrm{mmol}, 84 \%)$.

Flash column chromatography was performed using a mixture of hexane/ethyl acetate 4:1 as an eluent to provide the corresponding amide product.

White solid, mp 106-107 ${ }^{\circ} \mathrm{C} .{ }^{1} \mathrm{H}-\mathrm{NMR}\left(500 \mathrm{MHz}, \mathrm{CDCl}_{3}\right): \delta 2.41$ (s, 3H, Me), 6.85-6.90 $\left(\mathrm{m}, 2 \mathrm{H}, \mathrm{CH}_{\mathrm{Ar}}\right), 7.35-7.36\left(\mathrm{~m}, 2 \mathrm{H}, \mathrm{CH}_{\mathrm{Ar}}\right), 7.63-7.65\left(\mathrm{~m}, 1 \mathrm{H}, \mathrm{CH}_{\mathrm{Ar}}\right), 7.68\left(\mathrm{~s}, 1 \mathrm{H}, \mathrm{CH}_{\mathrm{Ar}}\right), 8.02$ (br s, 1H, NH), 8.29-8.33 (m, 1H, $\left.\mathrm{CH}_{\mathrm{Ar}}\right)$.

$\left.{ }^{13} \mathrm{C}^{1}{ }^{1} \mathrm{H}\right\}-\mathrm{NMR}\left(126 \mathrm{MHz}, \mathrm{CDCl}_{3}\right): \delta 21.4,103.4\left(\mathrm{~d}, J_{C F}=26.5 \mathrm{~Hz}\right), 103.6\left(\mathrm{~d}, J_{C F}=26.5 \mathrm{~Hz}\right)$, $111.2\left(\mathrm{dd}, J_{C F}=21.7 \mathrm{~Hz}, J_{C F}=3.6 \mathrm{~Hz}\right), 122.6\left(\mathrm{dd}, J_{C F}=10.5 \mathrm{~Hz}, J_{C F}=3.7 \mathrm{~Hz}\right), 123.1(\mathrm{~d}$, $\left.J_{C F}=9.3 \mathrm{~Hz}\right), 124.0,127.8,128.6,132.9,134.1,138.7,152.9\left(\mathrm{dd},{ }^{1} J_{C F}=246.4 \mathrm{~Hz}, J_{C F}=11.9 \mathrm{~Hz}\right)$, $158.6\left(\mathrm{dd},{ }^{1} J_{C F}=246.5 \mathrm{~Hz}, J_{C F}=11.4 \mathrm{~Hz}\right), 165.7$.

HRMS (TOF MS ES+): Calcd for $\mathrm{C}_{14} \mathrm{H}_{12} \mathrm{NOF}_{2}(\mathrm{M}+\mathrm{H})$ 248.0894. Found 248.0898.

3-chloro-N-(3,4-dimethoxyphenyl)benzamide 5e. The title compound was prepared starting with an appropriate 2-bromo-2,2-difluoroacetamide $1 \mathrm{~g}(310 \mathrm{mg}, 1.0 \mathrm{mmol}$, 1.0 equiv.), $\mathrm{KF}$ (116 mg, $2.0 \mathrm{mmol}, 2.0$ equiv.), $\mathrm{MgCl}_{2}$ (95 mg, $1.0 \mathrm{mmol}, 1.0$ equiv.), appropriate aryl boronic acid $2 \mathrm{a}(203 \mathrm{mg}, 1.3 \mathrm{mmol}, 1.3$ equiv.), the $\mathbf{L 1}(130 \mathrm{mg}, 0.2 \mathrm{mmol}$, 0.2 equiv.), $\mathrm{CuBr}_{2}(22.3 \mathrm{mg}, 0.1 \mathrm{mmol}, 0.1$ equiv.), and hexafluoropropanol $(0.12 \mathrm{mmol} / \mathrm{mL})$. The purification of the dry crude performed by column chromatography on silica gel provides the amide $5 \mathbf{e}(242 \mathrm{mg}, 0.83 \mathrm{mmol}, 83 \%)$.

Alternatively, the title compound was prepared starting with an appropriate 2-bromo2,2-difluoroacetamide 1 g (310 mg, $1.0 \mathrm{mmol}, 1.0$ equiv.), KF (116 mg, $2.0 \mathrm{mmol}, 2.0$ equiv.), $\mathrm{MgCl}_{2}$ (95 mg, $1.0 \mathrm{mmol}, 1.0$ equiv.), appropriate trialkoxysilane $3 \mathbf{i}(326 \mathrm{mg}, 1.4 \mathrm{mmol}$, 1.4 equiv.), the $\mathbf{L 1}\left(130 \mathrm{mg}, 0.2 \mathrm{mmol}, 0.2\right.$ equiv.), $\mathrm{CuBr}_{2}$ (22.3 mg, $0.1 \mathrm{mmol}, 0.1$ equiv.), and hexafluoropropanol $(0.12 \mathrm{mmol} / \mathrm{mL})$. The purification of the dry crude performed by column chromatography on silica gel provides the amide $5 \mathbf{e}(239 \mathrm{mg}, 0.82 \mathrm{mmol}, 82 \%)$.

Flash column chromatography was performed using a mixture of hexane/ethyl acetate 4:1 as an eluent to provide the corresponding amide product.

Light purple, solid mp 127-128 ${ }^{\circ} \mathrm{C} .{ }^{1} \mathbf{H}-\mathbf{N M R}\left(500 \mathrm{MHz}, \mathrm{CDCl}_{3}\right): \delta 3.79$ (s, 3H, OMe), $3.83(\mathrm{~s}, 3 \mathrm{H}, \mathrm{OMe}), 6.77\left(\mathrm{~d}, 1 \mathrm{H},{ }^{3} \mathrm{~J}=8.4 \mathrm{~Hz}, \mathrm{CH}_{\mathrm{Ar}}\right), 7.02\left(\mathrm{dd}, 1 \mathrm{H},{ }^{3} \mathrm{~J}=8.7 \mathrm{~Hz},{ }^{4} \mathrm{~J}=2.1 \mathrm{~Hz}\right.$, $\left.\mathrm{CH}_{\mathrm{Ar}}\right), 7.32\left(\mathrm{t}, 1 \mathrm{H},{ }^{3} J=7.9 \mathrm{~Hz}, \mathrm{CH}_{\mathrm{Ar}}\right), 7.36-7.37\left(\mathrm{~m}, 1 \mathrm{H}, \mathrm{CH}_{\mathrm{Ar}}\right), 7.44\left(\mathrm{dd}, 1 \mathrm{H},{ }^{3} J=8.0 \mathrm{~Hz}\right.$, $\left.{ }^{4} J=1.0 \mathrm{~Hz}, \mathrm{CH}_{\mathrm{Ar}}\right) .7 .70\left(\mathrm{~d}, 1 \mathrm{H},{ }^{3} \mathrm{~J}=7.0 \mathrm{~Hz}, \mathrm{CH}_{\mathrm{Ar}}\right), 7.81\left(\mathrm{~s}, 1 \mathrm{H}, \mathrm{CH}_{\mathrm{Ar}}\right), 8.26(\mathrm{~s}, 1 \mathrm{H}, \mathrm{NH})$.

$\left.{ }^{13} \mathrm{C}_{1}{ }^{1} \mathrm{H}\right\}$-NMR $\left(126 \mathrm{MHz}, \mathrm{CDCl}_{3}\right): \delta 55.7,55.9,105.2,111.1,112.6,125.1,127.3,129.9$, 131.1, 131.6, 134.7, 136.6, 146.1, 148.8, 164.5 .

HRMS (TOF MS ES+): Calcd for $\mathrm{C}_{15} \mathrm{H}_{15} \mathrm{NO}_{3} \mathrm{Cl}(\mathrm{M}+\mathrm{H})$ 292.0738. Found 292.0740.

3-methoxy-N-phenylbenzamide $5 f$. The title compound was prepared starting with an appropriate 2-bromo-2,2-difluoroacetamide $1 \mathrm{a}(250 \mathrm{mg}, 1.0 \mathrm{mmol}, 1.0$ equiv.), $\mathrm{KF}$ (116 mg, $2.0 \mathrm{mmol}, 2.0$ equiv.), $\mathrm{MgCl}_{2}$ (95 mg, $1.0 \mathrm{mmol}, 1.0$ equiv.), appropriate aryl boronic acid 2w (198 mg, $1.3 \mathrm{mmol}, 1.3$ equiv.), the $\mathbf{L 1}$ (130 mg, $0.2 \mathrm{mmol}, 0.2$ equiv.), $\mathrm{CuBr}_{2}$ (22.3 mg, $0.1 \mathrm{mmol}, 0.1$ equiv.), and hexafluoropropanol $(0.12 \mathrm{mmol} / \mathrm{mL})$. The purification of the dry crude performed by column chromatography on silica gel provides the amide $\mathbf{5 f}(207 \mathrm{mg}$, $0.91 \mathrm{mmol}, 91 \%)$.

Alternatively, the title compound was prepared starting with an appropriate 2-bromo2,2-difluoroacetamide 1a (250 mg, $1.0 \mathrm{mmol}, 1.0$ equiv.), KF (116 mg, $2.0 \mathrm{mmol}, 2.0$ equiv.), $\mathrm{MgCl}_{2}$ (95 mg, $1.0 \mathrm{mmol}, 1.0$ equiv.), appropriate trialkoxysilane $3 \mathbf{j}$ ( $378 \mathrm{mg}, 1.4 \mathrm{mmol}$, 1.4 equiv.), the $\mathbf{L} 1$ (130 mg, $0.2 \mathrm{mmol}, 0.2$ equiv.), $\mathrm{CuBr}_{2}(22.3 \mathrm{mg}, 0.1 \mathrm{mmol}, 0.1$ equiv.), and hexafluoropropanol $(0.12 \mathrm{mmol} / \mathrm{mL})$. The purification of the dry crude performed by column chromatography on silica gel provides the amide $5 \mathrm{f}(200 \mathrm{mg}, 0.88 \mathrm{mmol}, 88 \%)$. 
Alternatively, the title compound was prepared starting with an appropriate 2-bromo2,2-difluoroacetamide $1 \mathrm{a}(250 \mathrm{mg}, 1.0 \mathrm{mmol}, 1.0$ equiv.), $\mathrm{KF}$ (116 mg, $2.0 \mathrm{mmol}, 2.0$ equiv.), $\mathrm{MgCl}_{2}$ (95 mg, $1.0 \mathrm{mmol}, 1.0$ equiv.), aryl sulphonium salt $4 \mathbf{b}$ ( $410 \mathrm{mg}, 1.6 \mathrm{mmol}, 1.6$ equiv.), the $\mathbf{L} 2$ (202 $\mathrm{mg}, 0.25 \mathrm{mmol}, 0.25$ equiv.), [ $\mathrm{Ru}\left(\mathrm{p} \text {-cymene) } \mathrm{Cl}_{2}\right]_{2}$ (122 mg, $0.2 \mathrm{mmol}, 0.2$ equiv.), $\mathrm{CuBr}_{2}(67 \mathrm{mg}, 0.3 \mathrm{mmol}, 0.3$ equiv.), and hexafluoropropanol $(0.12 \mathrm{mmol} / \mathrm{mL})$. The purification of the dry crude performed by column chromatography on silica gel provides the amide $5 f(204 \mathrm{mg}, 0.90 \mathrm{mmol}, 90 \%)$.

Flash column chromatography was performed using a mixture of hexane/ethyl acetate 4:1 as an eluent to provide the corresponding amide product.

White solid, mp $116-117^{\circ} \mathrm{C} .{ }^{1} \mathrm{H}-\mathrm{NMR}\left(500 \mathrm{MHz}, \mathrm{CDCl}_{3}\right): \delta 3.78(\mathrm{~s}, 3 \mathrm{H}, \mathrm{OMe}), 7.02$ $\left(\mathrm{dd}, 1 \mathrm{H},{ }^{3} \mathrm{~J}=8.2 \mathrm{~Hz},{ }^{4} \mathrm{~J}=2.5 \mathrm{~Hz}, \mathrm{CH}_{\mathrm{Ar}}\right), 7.13\left(\mathrm{t}, 1 \mathrm{H},{ }^{3} \mathrm{~J}=7.5 \mathrm{~Hz}, \mathrm{CH}_{\mathrm{Ar}}\right), 7.28-7.40(\mathrm{~m}, 5 \mathrm{H}$, $\left.\mathrm{CH}_{\mathrm{Ar}}\right), 7.64\left(\mathrm{~d}, 2 \mathrm{H},{ }^{3} J=7.9 \mathrm{~Hz}, \mathrm{CH}_{\mathrm{Ar}}\right), 8.23(\mathrm{~s}, 1 \mathrm{H}, \mathrm{NH})$.

$\left.{ }^{13} \mathrm{C}_{\{}{ }^{1} \mathrm{H}\right\}$-NMR $\left(126 \mathrm{MHz}, \mathrm{CDCl}_{3}\right): \delta 55.3,112.3,117.9,118.8,120.3,124.5,128.9,129.6$, $136.3,137.9,159.8,165.8$.

HRMS (TOF MS ES+): Calcd for $\mathrm{C}_{14} \mathrm{H}_{14} \mathrm{NO}_{2}(\mathrm{M}+\mathrm{H})$ 228.1025. Found 228.1025.

4-fluoro-N-(m-tolyl)-3-(trifluoromethyl)benzamide $5 g$. The title compound was prepared starting with an appropriate 2-bromo-2,2-difluoroacetamide 1c $(264 \mathrm{mg}, 1.0 \mathrm{mmol}$, 1.0 equiv.), $\mathrm{KF}$ (116 mg, $2.0 \mathrm{mmol}, 2.0$ equiv.), $\mathrm{MgCl}_{2}$ (95 mg, $1.0 \mathrm{mmol}, 1.0$ equiv.), appropriate aryl boronic acid $\mathbf{2 p}(270 \mathrm{mg}, 1.3 \mathrm{mmol}, 1.3$ equiv.), the $\mathbf{L 1}(130 \mathrm{mg}, 0.2 \mathrm{mmol}$, 0.2 equiv.), $\mathrm{CuBr}_{2}(22.3 \mathrm{mg}, 0.1 \mathrm{mmol}, 0.1$ equiv.), and hexafluoropropanol $(0.12 \mathrm{mmol} / \mathrm{mL})$. The purification of the dry crude performed by column chromatography on silica gel provides the amide $5 \mathrm{~g}(267 \mathrm{mg}, 0.90 \mathrm{mmol}, 90 \%)$.

Flash column chromatography was performed using a mixture of hexane/ethyl acetate 5:1 as an eluent to provide the corresponding amide product.

Colorless solid, mp 121-122 ${ }^{\circ} \mathrm{C} .{ }^{1} \mathrm{H}-\mathrm{NMR}\left(500 \mathrm{MHz}, \mathrm{DMSO}-d_{6}\right): \delta 2.31$ (s, 3H, Me), $6.94\left(\mathrm{~d}, 1 \mathrm{H},{ }^{3} \mathrm{~J}=7.4 \mathrm{~Hz}, \mathrm{CH}_{\mathrm{Ar}}\right), 7.24\left(\mathrm{~d}, 1 \mathrm{H},{ }^{3} \mathrm{~J}=8.2 \mathrm{~Hz}, \mathrm{CH}_{\mathrm{Ar}}\right), 7.56\left(\mathrm{~d}, 2 \mathrm{H},{ }^{3} \mathrm{~J}=8.3 \mathrm{~Hz}\right.$, $\left.\mathrm{CH}_{\mathrm{Ar}}\right), 7.60\left(\mathrm{~s}, 1 \mathrm{H}, \mathrm{CH}_{\mathrm{Ar}}\right), 8.33-8.36\left(\mathrm{~m}, 2 \mathrm{H}, \mathrm{CH}_{\mathrm{Ar}}\right), 10.36(\mathrm{~s}, 1 \mathrm{H}, \mathrm{NH})$,

${ }^{13} \mathrm{C}\left\{{ }^{1} \mathrm{H}\right\}$-NMR $\left(126 \mathrm{MHz}, \mathrm{DMSO}-d_{6}\right): \delta 21.2,116.5\left(\mathrm{dd}, J_{C F}=33.5 \mathrm{~Hz}, J_{C F}=12.1 \mathrm{~Hz}\right)$, $117.5\left(\mathrm{~d}, J_{C F}=20.8 \mathrm{~Hz}\right), 117.7,121.1,122.4\left(\mathrm{q},{ }^{1} J_{C F}=272.3 \mathrm{~Hz}, \mathrm{CF}_{3}\right), 124.8,126.9,128.5,131.7$ $\left(\mathrm{d}, J_{C F}=3.1 \mathrm{~Hz}\right), 135.1\left(\mathrm{~d}, J_{C F}=9.4 \mathrm{~Hz}\right), 137.9,138.7,160.6\left(\mathrm{~d},{ }^{1} J_{C F}=257.0 \mathrm{~Hz}\right), 163.0$.

HRMS (TOF MS ES+): Calcd for $\mathrm{C}_{15} \mathrm{H}_{12} \mathrm{NOF}_{4}(\mathrm{M}+\mathrm{H})$ 298.0861. Found 298.0855.

$\mathrm{N}$-(p-tolyl)-2-(trifluoromethoxy)benzamide $5 \mathrm{~h}$. The title compound was prepared starting with an appropriate 2-bromo-2,2-difluoroacetamide $\mathbf{1 b}(264 \mathrm{mg}, 1.0 \mathrm{mmol}$, 1.0 equiv.), $\mathrm{KF}$ (116 mg, $2.0 \mathrm{mmol}, 2.0$ equiv.), $\mathrm{MgCl}_{2}$ ( $95 \mathrm{mg}, 1.0 \mathrm{mmol}, 1.0$ equiv.), appropriate aryl boronic acid $\mathbf{2 q}(268 \mathrm{mg}, 1.3 \mathrm{mmol}, 1.3$ equiv.), the $\mathbf{L 1}(130 \mathrm{mg}, 0.2 \mathrm{mmol}$, 0.2 equiv.), $\mathrm{CuBr}_{2}(22.3 \mathrm{mg}, 0.1 \mathrm{mmol}, 0.1$ equiv.), and hexafluoropropanol $(0.12 \mathrm{mmol} / \mathrm{mL})$. The purification of the dry crude performed by column chromatography on silica gel provides the amide $5 \mathrm{~h}(218 \mathrm{mg}, 0.74 \mathrm{mmol}, 74 \%)$.

Alternatively, the title compound was prepared starting with an appropriate 2-bromo2,2-difluoroacetamide $\mathbf{1 b}$ ( $264 \mathrm{mg}, 1.0 \mathrm{mmol}, 1.0$ equiv.), $\mathrm{KF}$ (116 mg, $2.0 \mathrm{mmol}, 2.0$ equiv.), $\mathrm{MgCl}_{2}$ (95 mg, $1.0 \mathrm{mmol}, 1.0$ equiv.), appropriate trialkoxysilane $3 \mathrm{~m}(395 \mathrm{mg}, 1.4 \mathrm{mmol}$, 1.4 equiv.), the $\mathbf{L 1}$ (130 mg, $0.2 \mathrm{mmol}, 0.2$ equiv.), $\mathrm{CuBr}_{2}$ ( $22.3 \mathrm{mg}, 0.1 \mathrm{mmol}, 0.1$ equiv.), and hexafluoropropanol $(0.12 \mathrm{mmol} / \mathrm{mL})$. The purification of the dry crude performed by column chromatography on silica gel provides the amide $5 \mathrm{~h}(221 \mathrm{mg}, 0.75 \mathrm{mmol}, 75 \%)$.

Flash column chromatography was performed using a mixture of hexane/ethyl acetate 5:1 as an eluent to provide the corresponding amide product.

White solid, mp 108-109 ${ }^{\circ} \mathrm{C} .{ }^{1} \mathrm{H}-\mathrm{NMR}\left(500 \mathrm{MHz}, \mathrm{CDCl}_{3}\right): \delta 2.35$ (s, 3H, Me), $7.18(\mathrm{~d}$, $\left.2 \mathrm{H},{ }^{3} \mathrm{~J}=8.0 \mathrm{~Hz}, \mathrm{CH}_{\mathrm{Ar}}\right), 7.32\left(\mathrm{~d}, 1 \mathrm{H},{ }^{3} \mathrm{~J}=8.0 \mathrm{~Hz}, \mathrm{CH}_{\mathrm{Ar}}\right), 7.42\left(\mathrm{t}, 1 \mathrm{H},{ }^{3} J=7.5 \mathrm{~Hz}, \mathrm{CH}_{\mathrm{Ar}}\right)$, 7.51-7.55 (m, 3H, $\left.\mathrm{CH}_{\mathrm{Ar}}\right), 8.04\left(\mathrm{~d}, 1 \mathrm{H},{ }^{3} \mathrm{~J}=7.7 \mathrm{~Hz}, \mathrm{CH}_{\mathrm{Ar}}\right), 8.30(\mathrm{~s}, 1 \mathrm{H}, \mathrm{NH})$.

$\left.{ }^{13} \mathrm{C}^{1}{ }^{1} \mathrm{H}\right\}$-NMR $\left(126 \mathrm{MHz}, \mathrm{CDCl}_{3}\right): \delta 20.9,120.3\left(\mathrm{q},{ }^{1} J_{\mathrm{CF}}=261.0 \mathrm{~Hz}, \mathrm{OCF}_{3}\right), 120.4,121.2$, 127.6, 128.3, 129.6, 131.9, 132.6, 134.6, 135.0, 145.7, 162.1 .

HRMS (TOF MS ES+): Calcd for $\mathrm{C}_{15} \mathrm{H}_{13} \mathrm{NO}_{3} \mathrm{~F}_{3}(\mathrm{M}+\mathrm{H})$ 312.0847. Found 312.0848.

$\mathrm{N}$-(4-methoxyphenyl)-2-(trifluoromethoxy)benzamide 5i. The title compound was prepared starting with an appropriate 2-bromo-2,2-difluoroacetamide $1 \mathbf{f}(280 \mathrm{mg}, 1.0 \mathrm{mmol}$, 
1.0 equiv.), $\mathrm{KF}$ ( $116 \mathrm{mg}, 2.0 \mathrm{mmol}, 2.0$ equiv.), $\mathrm{MgCl}_{2}$ (95 mg, $1.0 \mathrm{mmol}, 1.0$ equiv.), appropriate aryl boronic acid $\mathbf{2 q}(268 \mathrm{mg}, 1.3 \mathrm{mmol}, 1.3$ equiv.), the $\mathbf{L 1}(130 \mathrm{mg}, 0.2 \mathrm{mmol}$, 0.2 equiv.), $\mathrm{CuBr}_{2}(22.3 \mathrm{mg}, 0.1 \mathrm{mmol}, 0.1$ equiv.), and hexafluoropropanol $(0.12 \mathrm{mmol} / \mathrm{mL})$. The purification of the dry crude performed by column chromatography on silica gel provides the amide $5 \mathbf{i}(239 \mathrm{mg}, 0.77 \mathrm{mmol}, 77 \%)$. The gram scale synthesis was performed on $10 \mathrm{mmol}$ of the starting 2-bromo-2,2-difluoroacetamide $\mathbf{1 f}$ and the amide $5 \mathbf{i}$ was prepared in $63 \%$ yield $(1.96 \mathrm{~g}, 0.63 \mathrm{mmol})$.

Alternatively, the title compound was prepared starting with an appropriate 2-bromo2,2-difluoroacetamide 1f ( $280 \mathrm{mg}, 1.0 \mathrm{mmol}, 1.0$ equiv.), KF (116 mg, $2.0 \mathrm{mmol}, 2.0$ equiv.), $\mathrm{MgCl}_{2}$ (95 mg, $1.0 \mathrm{mmol}, 1.0$ equiv.), appropriate trialckoxysilane $3 \mathrm{~m}$ (395 mg, $1.4 \mathrm{mmol}$, 1.4 equiv.), the $\mathbf{L 1}\left(130 \mathrm{mg}, 0.2 \mathrm{mmol}, 0.2\right.$ equiv.), $\mathrm{CuBr}_{2}$ ( $22.3 \mathrm{mg}, 0.1 \mathrm{mmol}, 0.1$ equiv.), and hexafluoropropanol $(0.12 \mathrm{mmol} / \mathrm{mL})$. The purification of the dry crude performed by column chromatography on silica gel provides the amide $5 \mathbf{i}(224 \mathrm{mg}, 0.72 \mathrm{mmol}, 72 \%)$.

Flash column chromatography was performed using a mixture of hexane/ethyl acetate 6:1 as an eluent to provide the corresponding amide product.

Pink solid, mp 117-118 ${ }^{\circ} \mathrm{C} .{ }^{1} \mathrm{H}-\mathrm{NMR}\left(500 \mathrm{MHz}, \mathrm{CDCl}_{3}\right): \delta 3.81$ (s, 3H, OMe), 6.88-6.92 $\left(\mathrm{m}, 2 \mathrm{H}, \mathrm{CH}_{\mathrm{Ar}}\right), 7.32\left(\mathrm{~d}, 1 \mathrm{H},{ }^{3} \mathrm{~J}=8.2 \mathrm{~Hz}, \mathrm{CH}_{\mathrm{Ar}}\right), 7.42\left(\mathrm{dt}, 1 \mathrm{H},{ }^{3} \mathrm{~J}=7.6 \mathrm{~Hz},{ }^{4} J=0.7 \mathrm{~Hz}, \mathrm{CH}_{\mathrm{Ar}}\right)$, $7.51-7.54\left(\mathrm{~m}, 3 \mathrm{H}, \mathrm{CH}_{\mathrm{Ar}}\right), 8.02\left(\mathrm{dd}, 1 \mathrm{H},{ }^{3} \mathrm{~J}=7.8 \mathrm{~Hz},{ }^{4} J=1.7 \mathrm{~Hz}, \mathrm{CH}_{\mathrm{Ar}}\right), 8.25(\mathrm{~s}, 1 \mathrm{H}, \mathrm{NH})$.

${ }^{13} \mathrm{C}\left\{{ }^{1} \mathrm{H}\right\}$-NMR $\left(126 \mathrm{MHz}, \mathrm{CDCl}_{3}\right): \delta 55.5,114.2,120.3\left(\mathrm{q},{ }^{1} J_{\mathrm{CF}}=260.4 \mathrm{~Hz}, \mathrm{OCF}_{3}\right), 121.2$, 122.2, 127.6, 128.3, 130.6, 131.8, 132.5, 145.7, 156.8, 162.1 .

HRMS (TOF MS ES+): Calcd for $\mathrm{C}_{15} \mathrm{H}_{13} \mathrm{NO}_{2} \mathrm{~F}_{3}(\mathrm{M}+\mathrm{H})$ 296.0904. Found 296.0898.

$\mathrm{N}$-(m-tolyl)-2-(trifluoromethyl)benzamide $5 \mathrm{j}$. The title compound was prepared starting with an appropriate 2-bromo-2,2-difluoroacetamide $1 \mathrm{c}(264 \mathrm{mg}, 1.0 \mathrm{mmol}, 1.0$ equiv.), $\mathrm{KF}$ (116 mg, $2.0 \mathrm{mmol}, 2.0$ equiv.), $\mathrm{MgCl}_{2}$ ( $95 \mathrm{mg}, 1.0 \mathrm{mmol}, 1.0$ equiv.), appropriate aryl boronic acid 20 ( $247 \mathrm{mg}, 1.3 \mathrm{mmol}, 1.3$ equiv.), the $\mathbf{~} \mathbf{1}$ ( $130 \mathrm{mg}, 0.2 \mathrm{mmol}, 0.2$ equiv.), $\mathrm{CuBr}_{2}$ (22.3 $\mathrm{mg}, 0.1 \mathrm{mmol}, 0.1$ equiv.), and hexafluoropropanol $(0.12 \mathrm{mmol} / \mathrm{mL})$. The purification of the dry crude performed by column chromatography on silica gel provides the amide $5 \mathbf{j}$ (239 mg, $0.65 \mathrm{mmol}, 65 \%$ ).

Alternatively, the title compound was prepared starting with an appropriate 2-bromo2,2-difluoroacetamide 1c (264 mg, $1.0 \mathrm{mmol}, 1.0$ equiv.), $\mathrm{KF}$ (116 mg, $2.0 \mathrm{mmol}, 2.0$ equiv.), $\mathrm{MgCl}_{2}$ (95 mg, $1.0 \mathrm{mmol}, 1.0$ equiv.), appropriate trialkoxysilane $3 \mathrm{~m}(372 \mathrm{mg}, 1.4 \mathrm{mmol}$, 1.4 equiv.), the $\mathbf{L 1}$ (130 mg, $0.2 \mathrm{mmol}, 0.2$ equiv.), $\mathrm{CuBr}_{2}$ (22.3 mg, $0.1 \mathrm{mmol}, 0.1$ equiv.), and hexafluoropropanol $(0.12 \mathrm{mmol} / \mathrm{mL})$. The purification of the dry crude performed by column chromatography on silica gel provides the amide $5 \mathbf{j}(224 \mathrm{mg}, 0.67 \mathrm{mmol}, 67 \%)$.

Flash column chromatography was performed using a mixture of hexane/ethyl acetate 5:1 as an eluent to provide the corresponding amide product.

Colorless solid, mp 120-121 ${ }^{\circ} \mathrm{C} .{ }^{1} \mathrm{H}-\mathrm{NMR}\left(500 \mathrm{MHz}, \mathrm{CDCl}_{3}\right): \delta 2.35$ (s, 3H, Me), 6.98 $\left(\mathrm{d}, 1 \mathrm{H},{ }^{3} \mathrm{~J}=7.57 \mathrm{~Hz}, \mathrm{CH}_{\mathrm{Ar}}\right), 7.22\left(\mathrm{t}, 1 \mathrm{H},{ }^{3} \mathrm{~J}=8.1 \mathrm{~Hz}, \mathrm{CH}_{\mathrm{Ar}}\right), 7.33\left(\mathrm{~d}, 1 \mathrm{H},{ }^{3} \mathrm{~J}=7.6 \mathrm{~Hz}, \mathrm{CH}_{\mathrm{Ar}}\right)$, $7.44\left(\mathrm{~s}, 1 \mathrm{H}, \mathrm{CH}_{\mathrm{Ar}}\right), 7.54-7.57\left(\mathrm{~m}, 3 \mathrm{H}, \mathrm{CH}_{\mathrm{Ar}}\right), 7.69-7.71\left(\mathrm{~m}, 3 \mathrm{H}, \mathrm{NH}, \mathrm{CH}_{\mathrm{Ar}}\right)$.

$\left.{ }^{13} \mathrm{C}^{1}{ }^{1} \mathrm{H}\right\}$-NMR $\left(126 \mathrm{MHz}, \mathrm{CDCl}_{3}\right): \delta 21.4,117.3,120.8,123.7\left(\mathrm{q},{ }^{1} J_{\mathrm{CF}}=276.1 \mathrm{~Hz}, \mathrm{CF}_{3}\right)$, $125.7,126.4\left(\mathrm{q}, J_{C F}=5.2 \mathrm{~Hz}, \mathrm{CF}_{3}\right), 127.1\left(\mathrm{q},{ }^{2} J_{C F}=31.5 \mathrm{~Hz}, \mathrm{CCF}_{3}\right), 128.5,128.9,130.0,132.1$, $135.7,137.4,139.0,165.7$.

HRMS (TOF MS ES+): Calcd for $\mathrm{C}_{15} \mathrm{H}_{13} \mathrm{NOF}_{3}(\mathrm{M}+\mathrm{H})$ 280.0957. Found 280.0949.

$N$-(4-chlorophenyl)-2-methylbenzamide $5 k$. The title compound was prepared starting with an appropriate 2-bromo-2,2-difluoroacetamide $1 \mathrm{~h}$ ( $285 \mathrm{mg}, 1.0 \mathrm{mmol}, 1.0$ equiv.), $\mathrm{KF}$ (116 mg, $2.0 \mathrm{mmol}, 2.0$ equiv.), $\mathrm{MgCl}_{2}$ ( $95 \mathrm{mg}, 1.0 \mathrm{mmol}, 1.0$ equiv.), appropriate aryl boronic acid 2c (177 mg, $1.3 \mathrm{mmol}, 1.3$ equiv.), the $\mathbf{L 1}\left(130 \mathrm{mg}, 0.2 \mathrm{mmol}, 0.2\right.$ equiv.), $\mathrm{CuBr}_{2}$ (22.3 $\mathrm{mg}, 0.1 \mathrm{mmol}, 0.1$ equiv.), and hexafluoropropanol $(0.12 \mathrm{mmol} / \mathrm{mL})$. The purification of the dry crude performed by column chromatography on silica gel provides the amide 5k (172 mg, $0.70 \mathrm{mmol}, 70 \%)$.

Alternatively, the title compound was prepared starting with an appropriate 2-bromo2,2-difluoroacetamide $1 \mathrm{~h}$ ( $285 \mathrm{mg}, 1.0 \mathrm{mmol}, 1.0$ equiv.), $\mathrm{KF}$ (116 mg, $2.0 \mathrm{mmol}, 2.0$ equiv.), $\mathrm{MgCl}_{2}$ (95 mg, $1.0 \mathrm{mmol}, 1.0$ equiv.), appropriate trialkoxysilane $3 \mathrm{c}(297 \mathrm{mg}, 1.4 \mathrm{mmol}$, 1.4 equiv.), the $\mathbf{L 1}$ (130 mg, $0.2 \mathrm{mmol}, 0.2$ equiv.), $\mathrm{CuBr}_{2}$ ( $22.3 \mathrm{mg}, 0.1 \mathrm{mmol}, 0.1$ equiv.), 
and hexafluoropropanol $(0.12 \mathrm{mmol} / \mathrm{mL})$. The purification of the dry crude performed by column chromatography on silica gel provides the amide $5 \mathbf{k}(182 \mathrm{mg}, 0.74 \mathrm{mmol}, 74 \%)$.

Flash column chromatography was performed using a mixture of hexane/ethyl acetate 3:1 as an eluent to provide the corresponding amide product.

White solid, mp 136-138 ${ }^{\circ} \mathrm{C} .{ }^{1} \mathrm{H}-\mathrm{NMR}\left(500 \mathrm{MHz}, \mathrm{CDCl}_{3}\right): \delta 2.41(\mathrm{~s}, 3 \mathrm{H}, \mathrm{Me}), 7.17(\mathrm{t}$, $\left.1 \mathrm{H},{ }^{3} \mathrm{~J}=7.2 \mathrm{~Hz}, \mathrm{CH}_{\mathrm{Ar}}\right), 7.20\left(\mathrm{~d}, 1 \mathrm{H},{ }^{3} \mathrm{~J}=7.2 \mathrm{~Hz}, \mathrm{CH}_{\mathrm{Ar}}\right), 7.25\left(\mathrm{~d}, 2 \mathrm{H},{ }^{3} \mathrm{~J}=8.7 \mathrm{~Hz}, \mathrm{CH}_{\mathrm{Ar}}\right)$, $7.31\left(\mathrm{dt}, 2 \mathrm{H},{ }^{3} \mathrm{~J}=7.6 \mathrm{~Hz},{ }^{4} J=0.8 \mathrm{~Hz}, \mathrm{CH}_{\mathrm{Ar}}\right), 7.36\left(\mathrm{~d}, 1 \mathrm{H},{ }^{3} \mathrm{~J}=7.2 \mathrm{~Hz}, \mathrm{CH}_{\mathrm{Ar}}\right), 7.50(\mathrm{~d}, 1 \mathrm{H}$, $\left.{ }^{3} \mathrm{~J}=8.4 \mathrm{~Hz}, \mathrm{CH}_{\mathrm{Ar}}\right), 7.87(\mathrm{br} \mathrm{s}, 1 \mathrm{H}, \mathrm{NH})$.

$\left.{ }^{13} \mathrm{C}_{1}{ }^{1} \mathrm{H}\right\}-N M R\left(126 \mathrm{MHz}, \mathrm{CDCl}_{3}\right): \delta 19.7,121.2,125.8,126.6,129.0,129.4,130.3,131.2$, $135.9,136.3,136.5,168.2$.

HRMS (TOF MS ES+): Calcd for $\mathrm{C}_{14} \mathrm{H}_{13} \mathrm{NOCl}(\mathrm{M}+\mathrm{H})$ 246.0690. Found 246.0686.

2-bromo-N-(3,4-dimethoxyphenyl)benzamide 5l. The title compound was prepared starting with an appropriate 2-bromo-2,2-difluoroacetamide $1 \mathrm{~g}(310 \mathrm{mg}, 1.0 \mathrm{mmol}$, 1.0 equiv.), $\mathrm{KF}$ (116 mg, $2.0 \mathrm{mmol}, 2.0$ equiv.), $\mathrm{MgCl}_{2}$ (95 mg, $1.0 \mathrm{mmol}, 1.0$ equiv.), appropriate aryl boronic acid $\mathbf{2 m}(261 \mathrm{mg}, 1.3 \mathrm{mmol}, 1.3$ equiv.), the $\mathbf{L 1}(130 \mathrm{mg}, 0.2 \mathrm{mmol}, 0.2$ equiv.), $\mathrm{CuBr}_{2}(22.3 \mathrm{mg}, 0.1 \mathrm{mmol}, 0.1$ equiv.), and hexafluoropropanol $(0.12 \mathrm{mmol} / \mathrm{mL})$. The purification of the dry crude performed by column chromatography on silica gel provides the amide 51 (185 mg, $0.55 \mathrm{mmol}, 55 \%)$. Flash column chromatography was performed using a mixture of hexane/ethyl acetate 2:1 as an eluent to provide the corresponding amide product.

Purple solid, mp 140-141 ${ }^{\circ} \mathrm{C} .{ }^{1} \mathrm{H}-\mathrm{NMR}\left(500 \mathrm{MHz}, \mathrm{CDCl}_{3}\right): \delta 3.83$ (s, 3H, OMe), 3.84 $(\mathrm{s}, 3 \mathrm{H}, \mathrm{OMe}), 6.79\left(\mathrm{~d}, 1 \mathrm{H},{ }^{3} \mathrm{~J}=8.7 \mathrm{~Hz}, \mathrm{CH}_{\mathrm{Ar}}\right), 7.00\left(\mathrm{dd}, 1 \mathrm{H},{ }^{3} \mathrm{~J}=8.4 \mathrm{~Hz},{ }^{4} \mathrm{~J}=2.6 \mathrm{~Hz}, \mathrm{CH}_{\mathrm{Ar}}\right)$, 7.22-7.26 (m, 1H, CH $\left.\mathrm{Ar}_{\mathrm{Ar}}\right), 7.29-7.32\left(\mathrm{~m}, 1 \mathrm{H}, \mathrm{CH}_{\mathrm{Ar}}\right), 7.41\left(\mathrm{~d}, 1 \mathrm{H},{ }^{4} \mathrm{~J}=2.3 \mathrm{~Hz}, \mathrm{CH}_{\mathrm{Ar}}\right), 7.51(\mathrm{dd}$, $\left.1 \mathrm{H},{ }^{3} \mathrm{~J}=7.6 \mathrm{~Hz},{ }^{4} \mathrm{~J}=1.6 \mathrm{~Hz}, \mathrm{CH}_{\mathrm{Ar}}\right), 7.55\left(\mathrm{~d}, 1 \mathrm{H},{ }^{3} \mathrm{~J}=8.3 \mathrm{~Hz}, \mathrm{CH}_{\mathrm{Ar}}\right), 7.96(\mathrm{~s}, 1 \mathrm{H}, \mathrm{NH})$.

$\left.{ }^{13} \mathrm{C}^{1}{ }^{1} \mathrm{H}\right\}$-NMR $\left(126 \mathrm{MHz}, \mathrm{CDCl}_{3}\right): \delta 55.8,56.0,104.8,111.2,112.0,119.2,127.5,129.4$, $131.2,131.4,133.3,137.7,146.0,148.9,165.5$.

HRMS (TOF MS ES+): Calcd for $\mathrm{C}_{15} \mathrm{H}_{15} \mathrm{NO}_{3} \mathrm{Br}(\mathrm{M}+\mathrm{H})$ 266.0809. Found 266.0793.

$\mathrm{N}$-(4-fluorophenyl)nicotinamide $5 \mathrm{~m}$. The title compound was prepared starting with an appropriate 2-bromo-2,2-difluoroacetamide $1 \mathbf{i}$ ( $268 \mathrm{mg}, 1.0 \mathrm{mmol}$, 1.0 equiv.), $\mathrm{KF}$ (116 mg, $2.0 \mathrm{mmol}, 2.0$ equiv.), $\mathrm{MgCl}_{2}$ (95 mg, $1.0 \mathrm{mmol}, 1.0$ equiv.), appropriate aryl boronic acid 2t $\left(160 \mathrm{mg}, 1.3 \mathrm{mmol}, 1.3\right.$ equiv.), the $\mathbf{L 1}$ (130 mg, $0.2 \mathrm{mmol}, 0.2$ equiv.), $\mathrm{CuBr}_{2}$ (22.3 mg, $0.1 \mathrm{mmol}, 0.1$ equiv.), and hexafluoropropanol $(0.12 \mathrm{mmol} / \mathrm{mL})$. The purification of the dry crude performed by column chromatography on silica gel provides the amide $5 \mathrm{~m}(173 \mathrm{mg}$, $0.80 \mathrm{mmol}, 80 \%$ ).

Alternatively, the title compound was prepared starting with an appropriate 2-bromo2,2-difluoroacetamide 1i (268 mg, $1.0 \mathrm{mmol}, 1.0$ equiv.), KF (116 mg, $2.0 \mathrm{mmol}, 2.0$ equiv.), $\mathrm{MgCl}_{2}$ (95 mg, $1.0 \mathrm{mmol}$, 1.0 equiv.), appropriate trialkoxysilane $3 \mathbf{o}(277 \mathrm{mg}, 1.4 \mathrm{mmol}$, 1.4 equiv.), the $\mathbf{L 1}$ (130 mg, $0.2 \mathrm{mmol}, 0.2$ equiv.), $\mathrm{CuBr}_{2}$ ( $22.3 \mathrm{mg}, 0.1 \mathrm{mmol}, 0.1$ equiv.), and hexafluoropropanol $(0.12 \mathrm{mmol} / \mathrm{mL})$. The purification of the dry crude performed by column chromatography on silica gel provides the amide $5 \mathrm{~m}(166 \mathrm{mg}, 0.77 \mathrm{mmol}, 77 \%)$.

Flash column chromatography was performed using a mixture of hexane/ethyl acetate 1:1 as an eluent to provide the corresponding amide product.

White solid, mp 130-131 ${ }^{\circ} \mathrm{C} .{ }^{1} \mathbf{H}-\mathbf{N M R}\left(500 \mathrm{MHz}, \mathrm{CDCl}_{3}\right): \delta 7.02\left(\mathrm{t}, 2 \mathrm{H},{ }^{3} \mathrm{~J}=8.7 \mathrm{~Hz}\right.$, $\left.\mathrm{CH}_{\mathrm{Ar}}\right), 7.34-7.37\left(\mathrm{~m}, 1 \mathrm{H}, \mathrm{CH}_{\mathrm{Ar}}\right), 7.55-7.58\left(\mathrm{~m}, 2 \mathrm{H}, \mathrm{CH}_{\mathrm{Ar}}\right), 8.15\left(\mathrm{~d}, 1 \mathrm{H},{ }^{3} \mathrm{~J}=8.3 \mathrm{~Hz}, \mathrm{CH}_{\mathrm{Ar}}\right)$, $8.66\left(\mathrm{dd}, 1 \mathrm{H},{ }^{3} \mathrm{~J}=4.7 \mathrm{~Hz},{ }^{4} \mathrm{~J}=1.4 \mathrm{~Hz}, \mathrm{CH}_{\mathrm{Ar}}\right), 8.80(\mathrm{~s}, 1 \mathrm{H}, \mathrm{NH}), 9.03\left(\mathrm{~s}, 1 \mathrm{H}, \mathrm{CH}_{\mathrm{Ar}}\right)$.

$\left.{ }^{13} \mathrm{C}_{1}{ }^{1} \mathrm{H}\right\}$-NMR $\left(126 \mathrm{MHz}, \mathrm{CDCl}_{3}\right): \delta 115.7\left(\mathrm{~d}, J_{\mathrm{CF}}=22.2 \mathrm{~Hz}\right), 122.5\left(\mathrm{~d}, J_{C F}=7.0 \mathrm{~Hz}\right)$, $123.7,130.6,133.5,135.6,147.9,152.2,159.7\left(\mathrm{~d},{ }^{1} J_{C F}=244.1 \mathrm{~Hz}\right), 164.1$.

HRMS (TOF MS ES+): Calcd for $\mathrm{C}_{15} \mathrm{H}_{12} \mathrm{NOF}_{3} \mathrm{Na}(\mathrm{M}+\mathrm{Na})$ 302.0769. Found 302.0769.

$\mathrm{N}$-(m-tolyl)thiophene-2-carboxamide $5 n$. The title compound was prepared starting with an appropriate 2-bromo-2,2-difluoroacetamide $1 \mathrm{c}$ ( $264 \mathrm{mg}, 1.0 \mathrm{mmol}, 1.0$ equiv.), KF (116 mg, $2.0 \mathrm{mmol}, 2.0$ equiv.), $\mathrm{MgCl}_{2}$ (95 mg, $1.0 \mathrm{mmol}, 1.0$ equiv.), appropriate aryl boronic acid $\mathbf{2 u}$ (166 mg, $1.3 \mathrm{mmol}, 1.3$ equiv.), the $\mathbf{L 1}$ ( $130 \mathrm{mg}, 0.2 \mathrm{mmol}, 0.2$ equiv.), $\mathrm{CuBr}_{2}$ (22.3 $\mathrm{mg}$, $0.1 \mathrm{mmol}, 0.1$ equiv.), and hexafluoropropanol $(0.12 \mathrm{mmol} / \mathrm{mL})$. The purification 
of the dry crude performed by column chromatography on silica gel provides the amide 5n (154 mg, $0.71 \mathrm{mmol}, 71 \%)$.

Alternatively, the title compound was prepared starting with an appropriate 2-bromo2,2-difluoroacetamide 1c (264 mg, $1.0 \mathrm{mmol}, 1.0$ equiv.), KF (116 mg, $2.0 \mathrm{mmol}, 2.0$ equiv.), $\mathrm{MgCl}_{2}$ (95 mg, $1.0 \mathrm{mmol}$, 1.0 equiv.), appropriate trialkoxysilane $3 \mathrm{p}(286 \mathrm{mg}, 1.4 \mathrm{mmol}$, 1.4 equiv.), the $\mathbf{L 1}$ (130 mg, $0.2 \mathrm{mmol}, 0.2$ equiv.), $\mathrm{CuBr}_{2}$ ( $22.3 \mathrm{mg}, 0.1 \mathrm{mmol}, 0.1$ equiv.), and hexafluoropropanol $(0.12 \mathrm{mmol} / \mathrm{mL})$. The purification of the dry crude performed by column chromatography on silica gel provides the amide $5 \mathbf{n}(169 \mathrm{mg}, 0.78 \mathrm{mmol}, 78 \%$ ).

Alternatively, the title compound was prepared starting with an appropriate 2-bromo2,2-difluoroacetamide 1c ( $264 \mathrm{mg}$, $1.0 \mathrm{mmol}, 1.0$ equiv.), $\mathrm{KF}$ (116 mg, $2.0 \mathrm{mmol}, 2.0$ equiv.), $\mathrm{MgCl}_{2}$ (95 mg, $1.0 \mathrm{mmol}, 1.0$ equiv.), aryl sulphonium salt $4 \mathrm{c}(470 \mathrm{mg}, 1.6 \mathrm{mmol}, 1.6$ equiv.), the $\mathbf{L} 2$ ( $202 \mathrm{mg}, 0.25 \mathrm{mmol}, 0.25$ equiv.), [ $\mathrm{Ru}\left(\mathrm{p} \text {-cymene) } \mathrm{Cl}_{2}\right]_{2}$ ( $122 \mathrm{mg}, 0.2 \mathrm{mmol}, 0.2$ equiv.), $\mathrm{CuBr}_{2}(67 \mathrm{mg}, 0.3 \mathrm{mmol}, 0.3$ equiv.), and hexafluoropropanol $(0.12 \mathrm{mmol} / \mathrm{mL})$. The purification of the dry crude performed by column chromatography on silica gel provides the amide 5 n (174 $\mathrm{mg}, 0.80 \mathrm{mmol}, 80 \%)$.

Flash column chromatography was performed using a mixture of hexane/ethyl acetate 6:1 as an eluent to provide the corresponding amide product.

Brownish solid, mp $160-161{ }^{\circ} \mathrm{C} .{ }^{1} \mathrm{H}-\mathrm{NMR}\left(500 \mathrm{MHz}, \mathrm{CDCl}_{3}\right): \delta 2.32(\mathrm{~s}, 3 \mathrm{H}, \mathrm{Me}), 6.94$ $\left(\mathrm{t}, 1 \mathrm{H},{ }^{3} \mathrm{~J}=7.8 \mathrm{~Hz}, \mathrm{CH}_{\mathrm{Ar}}\right), 7.01-7.08\left(\mathrm{~m}, 1 \mathrm{H}\right.$, Thiophene), $7.21\left(\mathrm{t}, 1 \mathrm{H},{ }^{3} \mathrm{~J}=7.8 \mathrm{~Hz}, \mathrm{CH}_{\mathrm{Ar}}\right), 7.39$ $\left(\mathrm{d}, 1 \mathrm{H},{ }^{3} \mathrm{~J}=8.1 \mathrm{~Hz}, \mathrm{CH}_{\mathrm{Ar}}\right), 7.47\left(\mathrm{~s}, 1 \mathrm{H}, \mathrm{CH}_{\mathrm{Ar}}\right), 7.51\left(\mathrm{~d}, 1 \mathrm{H},{ }^{3} \mathrm{~J}=4.8 \mathrm{~Hz}\right.$, Thiophene), $7.64(\mathrm{~d}$, $1 \mathrm{H},{ }^{3} \mathrm{~J}=3.6 \mathrm{~Hz}$, Thiophene).

${ }^{13} \mathrm{C}\left\{{ }^{1} \mathrm{H}\right\}-N M R\left(126 \mathrm{MHz}, \mathrm{CDCl}_{3}\right): \delta 21.4,117.4,121.0,125.4,127.8,128.4,128.8,130.7$, $137.5,138.9,139.4,160.1$.

HRMS (TOF MS ES+): Calcd for $\mathrm{C}_{12} \mathrm{H}_{12} \mathrm{NOS}(\mathrm{M}+\mathrm{H})$ 240.1394. Found 218.0640.

3,4-difluoro- $\mathrm{N}$-isopropylbenzamide 5o. The title compound was prepared starting with an appropriate 2-bromo-2,2-difluoroacetamide $1 \mathrm{k}$ (216 mg, $1.0 \mathrm{mmol}, 1.0$ equiv.), $\mathrm{KF}$ (116 mg, $2.0 \mathrm{mmol}, 2.0$ equiv.), $\mathrm{MgCl}_{2}$ (95 mg, $1.0 \mathrm{mmol}, 1.0$ equiv.), appropriate aryl boronic acid $\mathbf{2 g}$ (205 mg, $1.3 \mathrm{mmol}, 1.3$ equiv.), the $\mathbf{~} \mathbf{1}$ ( $130 \mathrm{mg}, 0.2 \mathrm{mmol}, 0.2$ equiv.), $\mathrm{CuBr}_{2}$ ( $22.3 \mathrm{mg}, 0.1 \mathrm{mmol}, 0.1$ equiv.), and hexafluoropropanol $(0.12 \mathrm{mmol} / \mathrm{mL})$. The purification of the dry crude performed by column chromatography on silica gel provides the amide 5 o (238 mg, $0.90 \mathrm{mmol}, 90 \%)$.

Alternatively, the title compound was prepared starting with an appropriate 2-bromo2,2-difluoroacetamide 1k (216 mg, $1.0 \mathrm{mmol}, 1.0$ equiv.), $\mathrm{KF}$ (116 mg, $2.0 \mathrm{mmol}, 2.0$ equiv.), $\mathrm{MgCl}_{2}$ (95 mg, $1.0 \mathrm{mmol}, 1.0$ equiv.), appropriate trialkoxysilane $3 \mathrm{~g}$ ( $386 \mathrm{mg}, 1.4 \mathrm{mmol}$, 1.4 equiv.), the $\mathbf{L} 1$ (130 mg, $0.2 \mathrm{mmol}, 0.2$ equiv.), $\mathrm{CuBr}_{2}$ ( $22.3 \mathrm{mg}, 0.1 \mathrm{mmol}, 0.1$ equiv.), and hexafluoropropanol $(0.12 \mathrm{mmol} / \mathrm{mL})$. The purification of the dry crude performed by column chromatography on silica gel provides the amide 50 (233 $\mathrm{mg}, 0.88 \mathrm{mmol}, 88 \%$ ).

Flash column chromatography was performed using a mixture of hexane/ethyl acetate 8:1 as an eluent to provide the corresponding amide product.

White solid, mp 105-106 ${ }^{\circ} \mathrm{C} .{ }^{1} \mathrm{H}-\mathrm{NMR}\left(500 \mathrm{MHz}, \mathrm{CDCl}_{3}\right): \delta 1.14\left(\mathrm{~d}, 6 \mathrm{H},{ }^{3} \mathrm{~J}=6.2 \mathrm{~Hz}\right.$, $\mathrm{Me}), 4.08(\mathrm{~m}, 1 \mathrm{H}, \mathrm{CH}), 7.49-7.54\left(\mathrm{~m}, 1 \mathrm{H}, \mathrm{CH}_{\mathrm{Ar}}\right), 7.73-7374\left(\mathrm{~m}, 1 \mathrm{H}, \mathrm{CH}_{\mathrm{Ar}}\right), 7.87-7.91(\mathrm{~m}, 1 \mathrm{H}$, $\left.\mathrm{CH}_{\mathrm{Ar}}\right), 8.32\left(\mathrm{~d}, 1 \mathrm{H},{ }^{3} J=6.8 \mathrm{~Hz}, \mathrm{NH}\right)$.

$\left.{ }^{13} \mathrm{C}_{1}{ }^{1} \mathrm{H}\right\}$-NMR $\left(126 \mathrm{MHz}, \mathrm{CDCl}_{3}\right): \delta 22.2,41.3,116.6\left(\mathrm{~d}, J_{C F}=18.3 \mathrm{~Hz}\right), 117.3(\mathrm{~d}$, $\left.J_{C F}=17.5 \mathrm{~Hz}\right), 124.7(\mathrm{~m}), 132.2(\mathrm{~m}), 149.1\left(\mathrm{dd},{ }^{1} J_{C F}=247.3 \mathrm{~Hz},{ }^{1} J_{C F}=12.3 \mathrm{~Hz}\right), 151.2(\mathrm{dd}$, $\left.{ }^{1} J_{C F}=250.2 \mathrm{~Hz},{ }^{1} J_{C F}=12.3 \mathrm{~Hz}\right), 163.0$.

HRMS (TOF MS ES+): Calcd for $\mathrm{C}_{13} \mathrm{H}_{19} \mathrm{NOCl}(\mathrm{M}+\mathrm{H})$ 240.1157. Found 240.1155.

4-chloro- $\mathrm{N}, \mathrm{N}$-diisopropylbenzamide $5 p$. The title compound was prepared starting with an appropriate 2-bromo-2,2-difluoroacetamide 11 ( $258 \mathrm{mg}, 1.0 \mathrm{mmol}, 1.0$ equiv.), KF (116 mg, $2.0 \mathrm{mmol}, 2.0$ equiv.), $\mathrm{MgCl}_{2}$ (95 mg, $1.0 \mathrm{mmol}, 1.0$ equiv.), appropriate aryl boronic acid $\mathbf{2 j}$ ( $203 \mathrm{mg}, 1.3 \mathrm{mmol}, 1.3$ equiv.), the $\mathbf{L 1}$ (130 mg, $0.2 \mathrm{mmol}, 0.2$ equiv.), $\mathrm{CuBr}_{2}$ (22.3 mg, $0.1 \mathrm{mmol}, 0.1$ equiv.), and hexafluoropropanol $(0.12 \mathrm{mmol} / \mathrm{mL})$. The purification of the dry crude performed by column chromatography on silica gel provides the amide $5 \mathbf{p}$ ( $263 \mathrm{mg}$, $0.91 \mathrm{mmol}, 91 \%$ ). 
Alternatively, the title compound was prepared starting with an appropriate 2-bromo2,2-difluoroacetamide 11 ( $258 \mathrm{mg}, 1.0 \mathrm{mmol}, 1.0$ equiv.), $\mathrm{KF}$ (116 mg, $2.0 \mathrm{mmol}, 2.0$ equiv.), $\mathrm{MgCl}_{2}$ (95 mg, $1.0 \mathrm{mmol}, 1.0$ equiv.), appropriate trialkoxysilane $3 \mathrm{~h}(326 \mathrm{mg}, 1.4 \mathrm{mmol}$, 1.4 equiv.), the $\mathbf{L} 1$ (130 mg, $0.2 \mathrm{mmol}, 0.2$ equiv.), $\mathrm{CuBr}_{2}$ ( $22.3 \mathrm{mg}, 0.1 \mathrm{mmol}, 0.1$ equiv.), and hexafluoropropanol $(0.12 \mathrm{mmol} / \mathrm{mL})$. The purification of the dry crude performed by column chromatography on silica gel provides the amide $5 \mathrm{p}$ ( $266 \mathrm{mg}, 0.92 \mathrm{mmol}, 92 \%$ ).

Flash column chromatography was performed using a mixture of hexane/ethyl acetate 8:1 as an eluent to provide the corresponding amide product.

White solid, mp 129-130 ${ }^{\circ} \mathrm{C} .{ }^{1} \mathrm{H}-\mathrm{NMR}\left(500 \mathrm{MHz}, \mathrm{CDCl}_{3}\right): \delta 1.15$ (s, 6H, Me), 1.48 (s, $6 \mathrm{H}, \mathrm{Me}), 3.58(\mathrm{~m}, 2 \mathrm{H}, 2 \mathrm{xCH}), 7.24\left(\mathrm{dt}, 2 \mathrm{H},{ }^{3} \mathrm{~J}=8.5 \mathrm{~Hz},{ }^{4} \mathrm{~J}=2.0 \mathrm{~Hz}, \mathrm{CH}_{\mathrm{Ar}}\right), 7.34(\mathrm{dt}, 2 \mathrm{H}$, $\left.{ }^{3} J=8.3 \mathrm{~Hz},{ }^{4} J=1.7 \mathrm{~Hz}, \mathrm{CH}_{\mathrm{Ar}}\right)$.

${ }^{13} \mathrm{C}\left\{{ }^{1} \mathrm{H}\right\}$-NMR $\left(126 \mathrm{MHz}, \mathrm{CDCl}_{3}\right): \delta 20.7,127.1,128.7,134.6,137.2,169.9$.

HRMS (TOF MS ES+): Calcd for $\mathrm{C}_{13} \mathrm{H}_{19} \mathrm{NOCl}(\mathrm{M}+\mathrm{H})$ 240.1157. Found 240.1155.

2,3,4-trifluoro- $\mathrm{N}, \mathrm{N}$-diisopropylbenzamide $5 q$. Alternatively, the title compound was prepared starting with an appropriate 2-bromo-2,2-difluoroacetamide 11 (258 mg, $1.0 \mathrm{mmol}$, 1.0 equiv.), $\mathrm{KF}$ (116 mg, $2.0 \mathrm{mmol}, 2.0$ equiv.), $\mathrm{MgCl}_{2}$ (95 mg, $1.0 \mathrm{mmol}, 1.0$ equiv.), appropriate aryl boronic acid $\mathbf{2 h}(229 \mathrm{mg}, 1.3 \mathrm{mmol}, 1.3$ equiv.), the $\mathbf{L 1}(130 \mathrm{mg}, 0.2 \mathrm{mmol}$, 0.2 equiv.), $\mathrm{CuBr}_{2}(22.3 \mathrm{mg}, 0.1 \mathrm{mmol}, 0.1$ equiv.), and hexafluoropropanol $(0.12 \mathrm{mmol} / \mathrm{mL})$. The purification of the dry crude performed by column chromatography on silica gel provides the amide $5 \mathrm{q}(215 \mathrm{mg}, 0.83 \mathrm{mmol}, 83 \%)$.

Flash column chromatography was performed using a mixture of hexane/ethyl acetate 11:1 as an eluent to provide the corresponding amide product.

White solid, mp $48-50{ }^{\circ} \mathrm{C} .{ }^{1} \mathrm{H}-\mathrm{NMR}\left(500 \mathrm{MHz}, \mathrm{CDCl}_{3}\right): \delta 1.10(\mathrm{~m}, 6 \mathrm{H}, 2 \mathrm{xMe}), 1.50(\mathrm{~s}$, $3 \mathrm{H}, \mathrm{Me}), 1.52$ (s, 3H, Me), 3.50-3.53 (m, 1H, NCH), 3.65-3.68 53 (m, 1H, NCH), 6.97-7.00 $\left(\mathrm{m}, 2 \mathrm{H}, \mathrm{CH}_{\mathrm{Ar}}\right)$.

$\left.{ }^{13} \mathrm{C}^{1}{ }^{1} \mathrm{H}\right\}$-NMR $\left(126 \mathrm{MHz}, \mathrm{CDCl}_{3}\right): \delta 20.5(\mathrm{~m}), 46.2,51.3,112.9\left(\mathrm{dd}, J_{\mathrm{CF}}=18.0 \mathrm{~Hz}\right.$, $\left.J_{C F}=3.3 \mathrm{~Hz}\right), 121.3(\mathrm{~m}), 124.3\left(\mathrm{dd}, J_{C F}=16.4 \mathrm{~Hz}, J_{C F}=2.2 \mathrm{~Hz}\right), 139.7\left(\mathrm{dt},{ }^{1} J_{C F}=253.7 \mathrm{~Hz}\right.$, $\left.J_{C F}=15.2 \mathrm{~Hz}\right), 147.2\left(\mathrm{ddd},{ }^{1} J_{C F}=249.8 \mathrm{~Hz}, J_{C F}=10.9 \mathrm{~Hz}, J_{C F}=3.1 \mathrm{~Hz}\right), 151.2(\mathrm{ddd}$, $\left.{ }^{1} J_{C F}=251.3 \mathrm{~Hz}, J_{C F}=9.8 \mathrm{~Hz}, J_{C F}=2.4 \mathrm{~Hz}\right), 163.5$.

HRMS (TOF MS ES+): Calcd for $\mathrm{C}_{13} \mathrm{H}_{17} \mathrm{NOF}_{3}(\mathrm{M}+\mathrm{H})$ 260.1262. Found 260.1262.

$\mathrm{N}$-cyclohexyl-2-fluorobenzamide $5 \mathrm{r}$. The title compound was prepared starting with an appropriate 2-bromo-2,2-difluoroacetamide $1 p$ ( $258 \mathrm{mg}, 1.0 \mathrm{mmol}, 1.0$ equiv.), KF (116 mg, $2.0 \mathrm{mmol}, 2.0$ equiv.), $\mathrm{MgCl}_{2}$ (95 mg, $1.0 \mathrm{mmol}, 1.0$ equiv.), appropriate aryl boronic acid $\mathbf{2 i}$ ( $203 \mathrm{mg}, 1.3 \mathrm{mmol}, 1.3$ equiv.), the $\mathbf{L 1}$ ( $130 \mathrm{mg}, 0.2 \mathrm{mmol}, 0.2$ equiv.), $\mathrm{CuBr}_{2}$ (22.3 $\mathrm{mg}, 0.1 \mathrm{mmol}, 0.1$ equiv.), and hexafluoropropanol $(0.12 \mathrm{mmol} / \mathrm{mL})$. The purification of the dry crude performed by column chromatography on silica gel provides the amide $5 r$ (188 mg, $0.85 \mathrm{mmol}, 85 \%$ ).

Alternatively, the title compound was prepared starting with an appropriate 2-bromo2,2-difluoroacetamide 1p (258 mg, $1.0 \mathrm{mmol}, 1.0$ equiv.), $\mathrm{KF}$ (116 mg, $2.0 \mathrm{mmol}, 2.0$ equiv.), $\mathrm{MgCl}_{2}$ (95 mg, $1.0 \mathrm{mmol}$, 1.0 equiv.), appropriate potassium trifluoro(2-fluorophenyl)borate (263 mg, $1.3 \mathrm{mmol}, 1.3$ equiv.), the $\mathbf{L 1}$ (130 mg, $0.2 \mathrm{mmol}, 0.2$ equiv.), $\mathrm{CuBr}_{2}$ (22.3 mg, $0.1 \mathrm{mmol}, 0.1$ equiv.), and hexafluoropropanol $(0.12 \mathrm{mmol} / \mathrm{mL})$. The purification of the dry crude performed by column chromatography on silica gel provides the amide $5 \mathrm{r}$ (192 mg, $0.87 \mathrm{mmol}, 87 \%$ ).

Flash column chromatography was performed using a mixture of hexane/ethyl acetate 10:1 as an eluent to provide the corresponding amide product.

Colorless solid, $\mathrm{mp} 45-46{ }^{\circ} \mathrm{C} .{ }^{1} \mathrm{H}-\mathrm{NMR}\left(500 \mathrm{MHz}, \mathrm{CDCl}_{3}\right): \delta 1.19-1.29(\mathrm{~m}, 3 \mathrm{H}, \mathrm{Cy})$, 1.36-1.46 (m, 2H, Cy), 1.58-1.62 (m, 1H, Cy), 1.69-1.73 (m, 2H, Cy), 1.98-2.01 (m, 2H, Cy), $3.98-4.00(\mathrm{~m}, 1 \mathrm{H}, \mathrm{Cy}), 6.61(\mathrm{~s}, 1 \mathrm{H}, \mathrm{NH}), 7.04-7.08\left(\mathrm{~m}, 1 \mathrm{H}, \mathrm{CH}_{\mathrm{Ar}}\right), 7.19-7.22\left(\mathrm{~m}, 1 \mathrm{H}, \mathrm{CH}_{\mathrm{Ar}}\right)$, 7.38-7.42 (m, $\left.1 \mathrm{H}, \mathrm{CH}_{\mathrm{Ar}}\right), 8.03\left(\mathrm{dd}, 1 \mathrm{H},{ }^{3} \mathrm{~J}=7.9 \mathrm{~Hz},{ }^{4} J=1.8 \mathrm{~Hz}, \mathrm{CH}_{\mathrm{Ar}}\right)$.

$\left.{ }^{13} \mathrm{C}_{1}{ }^{1} \mathrm{H}\right\}$-NMR $\left(126 \mathrm{MHz}, \mathrm{CDCl}_{3}\right): \delta 24.7,25.5,32.9,48.5,115.8\left(\mathrm{~d}, J_{C F}=23.6 \mathrm{~Hz}\right)$, $121.5\left(\mathrm{~d}, J_{C F}=11.0 \mathrm{~Hz}\right), 124.6\left(\mathrm{~d}, J_{C F}=2.7 \mathrm{~Hz}\right), 131.9,133.0\left(\mathrm{~d}, J_{C F}=9.0 \mathrm{~Hz}\right), 160.4(\mathrm{~d}$, $\left.{ }^{1} J_{C F}=246.0 \mathrm{~Hz}, \mathrm{CF}\right), 162.1\left(\mathrm{~d}, J_{C F}=2.4 \mathrm{~Hz}\right)$.

HRMS (TOF MS ES+): Calcd for $\mathrm{C}_{13} \mathrm{H}_{17} \mathrm{NOF}(\mathrm{M}+\mathrm{H})$ 222.1294. Found 222.1294. 
$N$-cyclohexyl-N-methyl-[1,1'-biphenyl]-4-carboxamide 5s. The title compound was prepared starting with an appropriate 2-bromo-2,2-difluoroacetamide 10 ( $270 \mathrm{mg}, 1.0 \mathrm{mmol}$, 1.0 equiv.), $\mathrm{KF}$ (116 mg, $2.0 \mathrm{mmol}, 2.0$ equiv.), $\mathrm{MgCl}_{2}$ (95 mg, $1.0 \mathrm{mmol}, 1.0$ equiv.), appropriate aryl boronic acid $\mathbf{2 e}(257 \mathrm{mg}, 1.3 \mathrm{mmol}, 1.3$ equiv.), the $\mathbf{L 1}(130 \mathrm{mg}, 0.2 \mathrm{mmol}$, 0.2 equiv.), $\mathrm{CuBr}_{2}(22.3 \mathrm{mg}, 0.1 \mathrm{mmol}, 0.1$ equiv.), and hexafluoropropanol $(0.12 \mathrm{mmol} / \mathrm{mL})$. The purification of the dry crude performed by column chromatography on silica gel provides the amide $5 \mathrm{~s}(255 \mathrm{mg}, 0.87 \mathrm{mmol}, 87 \%)$.

Alternatively, the title compound was prepared starting with an appropriate 2-bromo2,2-difluoroacetamide 10 ( $270 \mathrm{mg}, 1.0 \mathrm{mmol}, 1.0$ equiv.), $\mathrm{KF}$ (116 mg, $2.0 \mathrm{mmol}, 2.0$ equiv.), $\mathrm{MgCl}_{2}$ (95 mg, $1.0 \mathrm{mmol}$, 1.0 equiv.), appropriate trialkoxysilane $3 \mathrm{e}(442 \mathrm{mg}, 1.4 \mathrm{mmol}$, 1.4 equiv.), the $\mathbf{L} 1$ (130 mg, $0.2 \mathrm{mmol}, 0.2$ equiv.), $\mathrm{CuBr}_{2}$ ( $22.3 \mathrm{mg}, 0.1 \mathrm{mmol}, 0.1$ equiv.), and hexafluoropropanol $(0.12 \mathrm{mmol} / \mathrm{mL})$. The purification of the dry crude performed by column chromatography on silica gel provides the amide $5 \mathrm{~s}(243 \mathrm{mg}, 0.83 \mathrm{mmol}, 83 \%$ ).

Flash column chromatography was performed using a mixture of hexane/ethyl acetate 8:1 as an eluent to provide the corresponding amide product.

Colorless solid, mp $105-106{ }^{\circ} \mathrm{C} .{ }^{1} \mathbf{H}-\mathrm{NMR}\left(500 \mathrm{MHz}, \mathrm{CDCl}_{3}\right): \delta 1.07-1.09(\mathrm{~m}, 2 \mathrm{H}$, Cy), 1.47-1.56 (m, 4H, Cy), 1.72-1.82 (m, 4H, Cy), 2.83, 3.00 (s, 3H, Me cis/trans), 3.54, 4.51 (s, $1 \mathrm{H}, \mathrm{Cy}$ cis/trans), 7.33-7.36 (m, $\left.1 \mathrm{H}, \mathrm{CH}_{\mathrm{Ar}}\right), 7.42-7.45\left(\mathrm{~m}, 4 \mathrm{H}, \mathrm{CH}_{\mathrm{Ar}}\right), 7.60-7.61(\mathrm{~m}, 4 \mathrm{H}$, $\mathrm{CH}_{\mathrm{Ar}}$ ).

$\left.{ }^{13} \mathrm{C}_{\{}{ }^{1} \mathrm{H}\right\}$-NMR $\left(126 \mathrm{MHz}, \mathrm{CDCl}_{3}\right): \delta$ 25.0, 25.3, 25.4, 27.4, 29.4, 29.5, 30.7, 31.9, 52.7, 58.1, 126.5 126.7, 126.9, 127.1, 127.2, 127.5, 128.7, 135.8, 140.1, 141.8, 171.4.

HRMS (TOF MS ES+): Calcd for $\mathrm{C}_{20} \mathrm{H}_{24} \mathrm{NO}(\mathrm{M}+\mathrm{H})$ 294.1856. Found 294.1858.

$N$-benzyl-3-methoxybenzamide $5 t$. The title compound was prepared starting with an appropriate 2-bromo-2,2-difluoroacetamide $1 \mathbf{u}$ ( $264 \mathrm{mg}$, $1.0 \mathrm{mmol}$, 1.0 equiv.), KF (116 mg, $2.0 \mathrm{mmol}, 2.0$ equiv.), $\mathrm{MgCl}_{2}$ (95 mg, $1.0 \mathrm{mmol}, 1.0$ equiv.), appropriate aryl boronic acid 2w (198 mg, $1.3 \mathrm{mmol}, 1.3$ equiv.), the $\mathbf{L 1}$ (130 mg, $0.2 \mathrm{mmol}$, 0.2 equiv.), $\mathrm{CuBr}_{2}$ (22.3 mg, $0.1 \mathrm{mmol}, 0.1$ equiv. $)$, and hexafluoropropanol $(0.12 \mathrm{mmol} / \mathrm{mL})$. The purification of the dry crude performed by column chromatography on silica gel provides the amide $5 \mathbf{t}(217 \mathrm{mg}$, $0.90 \mathrm{mmol}, 90 \%)$.

Alternatively, the title compound was prepared starting with an appropriate 2-bromo2,2-difluoroacetamide $1 \mathbf{u}$ (264 mg, $1.0 \mathrm{mmol}, 1.0$ equiv.), $\mathrm{KF}$ (116 mg, $2.0 \mathrm{mmol}, 2.0$ equiv.), $\mathrm{MgCl}_{2}$ (95 mg, $1.0 \mathrm{mmol}, 1.0$ equiv.), appropriate trialkoxysilane $3 \mathbf{j}(378 \mathrm{mg}, 1.4 \mathrm{mmol}$, 1.4 equiv.), the $\mathbf{L 1}\left(130 \mathrm{mg}, 0.2 \mathrm{mmol}, 0.2\right.$ equiv.), $\mathrm{CuBr}_{2}$ ( $22.3 \mathrm{mg}, 0.1 \mathrm{mmol}, 0.1$ equiv.), and hexafluoropropanol $(0.12 \mathrm{mmol} / \mathrm{mL})$. The purification of the dry crude performed by column chromatography on silica gel provides the amide $5 \mathbf{t}(210 \mathrm{mg}, 0.87 \mathrm{mmol}, 87 \%)$.

Alternatively, the title compound was prepared starting with an appropriate 2-bromo2,2-difluoroacetamide $1 \mathbf{u}$ (264 mg, $1.0 \mathrm{mmol}, 1.0$ equiv.), KF (116 mg, $2.0 \mathrm{mmol}, 2.0$ equiv.), $\mathrm{MgCl}_{2}$ (95 mg, $1.0 \mathrm{mmol}, 1.0$ equiv.), aryl sulphonium salt $4 \mathbf{b}$ ( $410 \mathrm{mg}, 1.6 \mathrm{mmol}, 1.6$ equiv.), the $\mathbf{L} 2$ (202 mg, $0.25 \mathrm{mmol}, 0.25$ equiv.), [ $\mathrm{Ru}\left(\mathrm{p} \text {-cymene) } \mathrm{Cl}_{2}\right]_{2}$ ( $122 \mathrm{mg}, 0.2 \mathrm{mmol}, 0.2$ equiv.), $\mathrm{CuBr}_{2}$ ( $67 \mathrm{mg}, 0.3 \mathrm{mmol}, 0.3$ equiv.), and hexafluoropropanol $(0.17 \mathrm{mmol} / \mathrm{mL})$. The purification of the dry crude performed by column chromatography on silica gel provides the amide $5 t$ (202 $\mathrm{mg}, 0.84 \mathrm{mmol}, 84 \%)$.

Alternatively, the title compound was prepared starting with an appropriate 2-bromo2,2-difluoroacetamide $1 \mathbf{u}(264 \mathrm{mg}, 1.0 \mathrm{mmol}, 1.0$ equiv.), $\mathrm{KF}$ (116 mg, $2.0 \mathrm{mmol}, 2.0$ equiv.), $\mathrm{MgCl}_{2}$ (95 mg, $1.0 \mathrm{mmol}, 1.0$ equiv.), appropriate potassium trifluoro(3-methoxyphenyl) borate ( $278 \mathrm{mg}, 1.3 \mathrm{mmol}, 1.3$ equiv.), the $\mathbf{L 1}\left(130 \mathrm{mg}, 0.2 \mathrm{mmol}, 0.2\right.$ equiv.), $\mathrm{CuBr}_{2}$ (22.3 mg, $0.1 \mathrm{mmol}, 0.1$ equiv. $)$, and hexafluoropropanol $(0.12 \mathrm{mmol} / \mathrm{mL})$. The purification of the dry crude performed by column chromatography on silica gel provides the amide $5 \mathbf{t}$ (193 mg, $0.80 \mathrm{mmol}, 80 \%)$.

Alternatively, the title compound was prepared starting with an appropriate 2-bromo2,2-difluoroacetamide $1 \mathbf{u}(264 \mathrm{mg}$, $1.0 \mathrm{mmol}, 1.0$ equiv.), $\mathrm{KF}(116 \mathrm{mg}, 2.0 \mathrm{mmol}, 2.0$ equiv.), $\mathrm{MgCl}_{2}$ (95 mg, $1.0 \mathrm{mmol}, 1.0$ equiv.), appropriate 2-(3-methoxyphenyl)-4,4,5,5-tetramethyl1,3,2-dioxaborolane ( $304 \mathrm{mg}, 1.3 \mathrm{mmol}, 1.3$ equiv.), the $\mathbf{L 1}$ (130 mg, $0.2 \mathrm{mmol}, 0.2$ equiv.), $\mathrm{CuBr}_{2}(22.3 \mathrm{mg}, 0.1 \mathrm{mmol}, 0.1$ equiv.), and hexafluoropropanol $(0.12 \mathrm{mmol} / \mathrm{mL})$. The 
purification of the dry crude performed by column chromatography on silica gel provides the amide $5 \mathbf{t}$ (200 $\mathrm{mg}, 0.83 \mathrm{mmol}, 83 \%$ ).

Alternatively, the title compound was prepared starting with an appropriate Nbenzyl-2,2-difluoro-2-iodoacetamide ( $311 \mathrm{mg}$, $1.0 \mathrm{mmol}$, 1.0 equiv.), $\mathrm{KF}(116 \mathrm{mg}, 2.0 \mathrm{mmol}$, 2.0 equiv.), $\mathrm{MgCl}_{2}$ (95 mg, $1.0 \mathrm{mmol}, 1.0$ equiv.), appropriate aryl boronic acid $\mathbf{2} \mathbf{w}$ (198 mg, $1.3 \mathrm{mmol}, 1.3$ equiv.), the $\mathbf{L 1}\left(130 \mathrm{mg}, 0.2 \mathrm{mmol}, 0.2\right.$ equiv.), $\mathrm{CuBr}_{2}(22.3 \mathrm{mg}, 0.1 \mathrm{mmol}$, 0.1 equiv.), and hexafluoropropanol $(0.12 \mathrm{mmol} / \mathrm{mL})$. The purification of the dry crude performed by column chromatography on silica gel provides the amide $5 \mathbf{t}$ ( $210 \mathrm{mg}$, $0.87 \mathrm{mmol}, 87 \%)$.

Flash column chromatography was performed using a mixture of hexane/ethyl acetate 7:1 as an eluent to provide the corresponding amide product.

White solid, mp 77-78 ${ }^{\circ} \mathrm{C} .{ }^{1} \mathrm{H}-\mathrm{NMR}\left(500 \mathrm{MHz}, \mathrm{CDCl}_{3}\right): \delta 2.36(\mathrm{~s}, 3 \mathrm{H}, \mathrm{Me}), 4.60(\mathrm{~d}, 2 \mathrm{H}$, $\left.{ }^{3} J=5.6 \mathrm{~Hz}, \mathrm{CH}_{2}\right), 6.78($ br s, $1 \mathrm{H}, \mathrm{NH}), 7.26-7.33\left(\mathrm{~m}, 7 \mathrm{H}, \mathrm{CH}_{\mathrm{Ar}}\right), 7.57\left(\mathrm{~d}, 1 \mathrm{H},{ }^{3} \mathrm{~J}=7.2 \mathrm{~Hz}\right.$, $\left.\mathrm{CH}_{\mathrm{Ar}}\right), 7.63\left(\mathrm{~s}, 1 \mathrm{H}, \mathrm{CH}_{\mathrm{Ar}}\right)$.

${ }^{13} \mathrm{C}\left\{{ }^{1} \mathrm{H}\right\}-\mathrm{NMR}\left(126 \mathrm{MHz}, \mathrm{CDCl}_{3}\right): \delta 21.2,43.9,123.9,127.4,127.7,127.8,128.3,128.6$, 132.1, 134.2, 138.3, 167.6.

HRMS (TOF MS ES+): Calcd for $\mathrm{C}_{15} \mathrm{H}_{16} \mathrm{NO}(\mathrm{M}+\mathrm{H})$ 226.1234. Found 226.1232.

$\mathrm{N}$-cyclohexyl-N-phenyl-4-(trifluoromethyl)benzamide $5 u$. The title compound was prepared starting with an appropriate 2-bromo-2,2-difluoroacetamide $1 \mathbf{n}(332 \mathrm{mg}, 1.0 \mathrm{mmol}$, 1.0 equiv.), KF (116 mg, $2.0 \mathrm{mmol}, 2.0$ equiv.), $\mathrm{MgCl}_{2}$ (95 mg, $1.0 \mathrm{mmol}, 1.0$ equiv.), appropriate aryl boronic acid $\mathbf{2 n}(247 \mathrm{mg}, 1.3 \mathrm{mmol}, 1.3$ equiv.), the $\mathbf{L 1}(130 \mathrm{mg}, 0.2 \mathrm{mmol}$, 0.2 equiv.), $\mathrm{CuBr}_{2}(22.3 \mathrm{mg}, 0.1 \mathrm{mmol}, 0.1$ equiv.), and hexafluoropropanol $(0.12 \mathrm{mmol} / \mathrm{mL})$. The purification of the dry crude performed by column chromatography on silica gel provides the amide $5 \mathbf{u}(312 \mathrm{mg}, 0.90 \mathrm{mmol}, 90 \%)$.

Alternatively, the title compound was prepared starting with an appropriate 2-bromo2,2-difluoroacetamide $1 \mathrm{n}$ (332 mg, $1.0 \mathrm{mmol}, 1.0$ equiv.), KF (116 mg, $2.0 \mathrm{mmol}, 2.0$ equiv.), $\mathrm{MgCl}_{2}$ (95 mg, $1.0 \mathrm{mmol}$, 1.0 equiv.), appropriate trialkoxysilane $3 \mathrm{k}$ (372 mg, $1.4 \mathrm{mmol}$, 1.4 equiv.), the $\mathbf{L 1}$ ( $130 \mathrm{mg}, 0.2 \mathrm{mmol}, 0.2$ equiv.), $\mathrm{CuBr}_{2}$ ( $22.3 \mathrm{mg}, 0.1 \mathrm{mmol}, 0.1$ equiv.), and hexafluoropropanol $(0.12 \mathrm{mmol} / \mathrm{mL})$. The purification of the dry crude performed by column chromatography on silica gel provides the amide $5 \mathbf{u}(312 \mathrm{mg}, 0.90 \mathrm{mmol}, 90 \%)$.

Alternatively, the title compound was prepared starting with an appropriate 2-bromo2,2-difluoroacetamide 1n (332 mg, $1.0 \mathrm{mmol}, 1.0$ equiv.), KF (116 mg, $2.0 \mathrm{mmol}, 2.0$ equiv.), $\mathrm{MgCl}_{2}$ (95 mg, $1.0 \mathrm{mmol}, 1.0$ equiv.), aryl sulphonium salt $4 \mathrm{a}$ ( $584 \mathrm{mg}, 1.6 \mathrm{mmol}, 1.6$ equiv.), the $\mathbf{L} 2$ (202 $\mathrm{mg}, 0.25 \mathrm{mmol}, 0.25$ equiv.), [ $\mathrm{Ru}\left(\mathrm{p} \text {-cymene) } \mathrm{Cl}_{2}\right]_{2}$ (122 $\mathrm{mg}, 0.2 \mathrm{mmol}, 0.2$ equiv.), $\mathrm{CuBr}_{2}(67 \mathrm{mg}, 0.3 \mathrm{mmol}, 0.3$ equiv.), and hexafluoropropanol $(0.12 \mathrm{mmol} / \mathrm{mL})$. The purification of the dry crude performed by column chromatography on silica gel provides the amide $5 \mathbf{u}(281 \mathrm{mg}, 0.81 \mathrm{mmol}, 81 \%)$.

Flash column chromatography was performed using a mixture of hexane/ethyl acetate 8:1 as an eluent to provide the corresponding amide product.

Colorless solid, mp 195-196 ${ }^{\circ} \mathrm{C} .{ }^{1} \mathrm{H}-\mathrm{NMR}\left(500 \mathrm{MHz}, \mathrm{CDCl}_{3}\right): \delta 0.97\left(\mathrm{tq}, 1 \mathrm{H},{ }^{3} \mathrm{~J}=13.4 \mathrm{~Hz}\right.$, $\left.{ }^{4} J=2.9 \mathrm{~Hz}, \mathrm{Cy}\right), 1.22\left(\mathrm{dq}, 2 \mathrm{H},{ }^{3} \mathrm{~J}=12.9 \mathrm{~Hz},{ }^{4} \mathrm{~J}=2.9 \mathrm{~Hz}, \mathrm{Cy}\right), 1.45\left(\mathrm{~d}, 2 \mathrm{H},{ }^{3} \mathrm{~J}=12.4 \mathrm{~Hz}, \mathrm{Cy}\right), 1.61$ $\left(\mathrm{d}, 1 \mathrm{H},{ }^{3} \mathrm{~J}=13.3 \mathrm{~Hz}, \mathrm{Cy}\right), 1.78\left(\mathrm{~d}, 2 \mathrm{H},{ }^{3} \mathrm{~J}=13.3 \mathrm{~Hz}, \mathrm{Cy}\right), 1.96\left(\mathrm{~d}, 2 \mathrm{H},{ }^{3} \mathrm{~J}=11.1 \mathrm{~Hz}, \mathrm{Cy}\right), 4.72(\mathrm{~s}$, $1 \mathrm{H}, \mathrm{Cy}), 7.00\left(\mathrm{~d}, 2 \mathrm{H},{ }^{3} \mathrm{~J}=6.9 \mathrm{~Hz}, \mathrm{CH}_{\mathrm{Ar}}\right), 7.19-7.20\left(\mathrm{~m}, 3 \mathrm{H}, \mathrm{CH}_{\mathrm{Ar}}\right), 7.31\left(\mathrm{~d}, 2 \mathrm{H},{ }^{3} \mathrm{~J}=7.3 \mathrm{~Hz}\right.$, $\left.\mathrm{CH}_{\mathrm{Ar}}\right), 7.36\left(\mathrm{~d}, 2 \mathrm{H},{ }^{3} \mathrm{~J}=6.9 \mathrm{~Hz}, \mathrm{CH}_{\mathrm{Ar}}\right)$.

${ }^{13} \mathrm{C}\left\{{ }^{1} \mathrm{H}\right\}$-NMR $\left(126 \mathrm{MHz}, \mathrm{CDCl}_{3}\right): \delta 25.3,25.8,31.5,55.3,123.7\left(\mathrm{q},{ }^{1} J_{\mathrm{CF}}=271.2 \mathrm{~Hz}\right.$, $\left.\mathrm{CF}_{3}\right), 124.6,127.8,128.3,128.6,130.4\left(\mathrm{q},{ }^{2} J_{C F}=28.0 \mathrm{~Hz}, \mathrm{CCF}_{3}\right), 130.6,139.2,140.8,169.1$.

HRMS (TOF MS ES+): Calcd for $\mathrm{C}_{20} \mathrm{H}_{21} \mathrm{NOF}_{3}(\mathrm{M}+\mathrm{H})$ 348.1581. Found 348.1575.

$N-((1 s, 3 s)$-adamantan-1-yl)-4-(trifluoromethyl)benzamide 5v. The title compound was prepared starting with an appropriate 2-bromo-2,2-difluoroacetamide 1q (308 mg, $1.0 \mathrm{mmol}, 1.0$ equiv.), $\mathrm{KF}$ (116 mg, $2.0 \mathrm{mmol}$, 2.0 equiv.), $\mathrm{MgCl}_{2}$ ( $95 \mathrm{mg}, 1.0 \mathrm{mmol}, 1.0$ equiv.), appropriate aryl boronic acid $\mathbf{2 n}(247 \mathrm{mg}, 1.3 \mathrm{mmol}, 1.3$ equiv.), the $\mathbf{L 1}(130 \mathrm{mg}, 0.2 \mathrm{mmol}$, 0.2 equiv.), $\mathrm{CuBr}_{2}(22.3 \mathrm{mg}, 0.1 \mathrm{mmol}, 0.1$ equiv.), and hexafluoropropanol $(0.12 \mathrm{mmol} / \mathrm{mL})$. The purification of the dry crude performed by column chromatography on silica gel provides the amide $5 \mathbf{v}(281 \mathrm{mg}, 0.87 \mathrm{mmol}, 87 \%)$. 
Alternatively, the title compound was prepared starting with an appropriate 2-bromo2,2-difluoroacetamide $1 q$ ( $308 \mathrm{mg}, 1.0 \mathrm{mmol}, 1.0$ equiv.), $\mathrm{KF}$ (116 mg, $2.0 \mathrm{mmol}, 2.0$ equiv.), $\mathrm{MgCl}_{2}$ (95 mg, $1.0 \mathrm{mmol}, 1.0$ equiv.), appropriate trialkoxysilane $3 \mathbf{k}(372 \mathrm{mg}, 1.4 \mathrm{mmol}$, 1.4 equiv.), the $\mathbf{L} 1$ (130 mg, $0.2 \mathrm{mmol}, 0.2$ equiv.), $\mathrm{CuBr}_{2}$ ( $22.3 \mathrm{mg}, 0.1 \mathrm{mmol}, 0.1$ equiv.), and hexafluoropropanol $(0.12 \mathrm{mmol} / \mathrm{mL})$. The purification of the dry crude performed by column chromatography on silica gel provides the amide $5 \mathbf{v}$ ( $291 \mathrm{mg}, 0.90 \mathrm{mmol}, 90 \%$ ).

Alternatively, the title compound was prepared starting with an appropriate 2-bromo2,2-difluoroacetamide 1q (308 mg, $1.0 \mathrm{mmol}, 1.0$ equiv.), $\mathrm{KF}$ (116 mg, $2.0 \mathrm{mmol}, 2.0$ equiv.), $\mathrm{MgCl}_{2}$ (95 mg, $1.0 \mathrm{mmol}, 1.0$ equiv.), appropriate potassium trifluoro(4-(trifluoromethyl) phenyl)borate ( $328 \mathrm{mg}, 1.3 \mathrm{mmol}, 1.3$ equiv.), the $\mathbf{L 1}$ ( $130 \mathrm{mg}, 0.2 \mathrm{mmol}, 0.2$ equiv.), $\mathrm{CuBr}_{2}$ (22.3 $\mathrm{mg}, 0.1 \mathrm{mmol}, 0.1$ equiv.), and hexafluoropropanol $(0.12 \mathrm{mmol} / \mathrm{mL})$. The purification of the dry crude performed by column chromatography on silica gel provides the amide $5 \mathbf{v}$ (271 $\mathrm{mg}, 0.84 \mathrm{mmol}, 84 \%)$.

Flash column chromatography was performed using a mixture of hexane/ethyl acetate 12:1 as an eluent to provide the corresponding amide product.

White solid, mp 158-159 ${ }^{\circ} \mathrm{C} .{ }^{1} \mathrm{H}-\mathrm{NMR}\left(500 \mathrm{MHz}, \mathrm{CDCl}_{3}\right): \delta 1.70$ (s, 6H, Adam), 2.11 (s, 9H, Adam), $5.91(\mathrm{~s}, 1 \mathrm{H}, \mathrm{NH}), 7.61\left(\mathrm{~d}, 2 \mathrm{H},{ }^{3} \mathrm{~J}=8.6 \mathrm{~Hz}, \mathrm{CH}_{\mathrm{Ar}}\right), 7.78\left(\mathrm{~d}, 2 \mathrm{H},{ }^{3} \mathrm{~J}=7.9 \mathrm{~Hz}\right.$, $\mathrm{CH}_{\mathrm{Ar}}$ ).

${ }^{13} \mathrm{C}\left\{{ }^{1} \mathrm{H}\right\}$-NMR $\left(126 \mathrm{MHz}, \mathrm{CDCl}_{3}\right): \delta 29.4,36.2,41.5,52.6,123.7\left(\mathrm{q},{ }^{1} J_{C F}=273.6 \mathrm{~Hz}\right.$, $\left.\mathrm{CF}_{3}\right), 125.4(\mathrm{~m}), 127.2,132.6\left(\mathrm{q},{ }^{2} J_{\mathrm{CF}}=31.1 \mathrm{~Hz}, \mathrm{CCF}_{3}\right), 139.3$.

HRMS (TOF MS ES+): Calcd for $\mathrm{C}_{18} \mathrm{H}_{21} \mathrm{NOF}_{3}(\mathrm{M}+\mathrm{H})$ 324.1583. Found 324.1575.

3-chloro-N-cyclopropylbenzamide 5w. The title compound was prepared starting with an appropriate 2-bromo-2,2-difluoroacetamide $1 \mathbf{r}$ ( $214 \mathrm{mg}, 1.0 \mathrm{mmol}, 1.0$ equiv.), KF (116 mg, $2.0 \mathrm{mmol}, 2.0$ equiv.), $\mathrm{MgCl}_{2}$ (95 mg, $1.0 \mathrm{mmol}, 1.0$ equiv.), appropriate aryl boronic acid 2k (203 mg, $1.3 \mathrm{mmol}, 1.3$ equiv.), the $\mathbf{L 1}$ (130 mg, $0.2 \mathrm{mmol}, 0.2$ equiv.), $\mathrm{CuBr}_{2}$ (22.3 $\mathrm{mg}, 0.1 \mathrm{mmol}, 0.1$ equiv.), and hexafluoropropanol $(0.12 \mathrm{mmol} / \mathrm{mL})$. The purification of the dry crude performed by column chromatography on silica gel provides the amide 5 w $(127 \mathrm{mg}, 0.65 \mathrm{mmol}, 65 \%)$.

Alternatively, the title compound was prepared starting with an appropriate 2-bromo2,2-difluoroacetamide $1 \mathrm{r}(214 \mathrm{mg}, 1.0 \mathrm{mmol}, 1.0$ equiv.), $\mathrm{KF}(116 \mathrm{mg}, 2.0 \mathrm{mmol}, 2.0$ equiv.), $\mathrm{MgCl}_{2}$ (95 mg, $1.0 \mathrm{mmol}, 1.0$ equiv.), appropriate trialkoxysilane $3 \mathbf{i}(326 \mathrm{mg}, 1.4 \mathrm{mmol}$, 1.4 equiv.), the $\mathbf{L 1}$ (130 mg, $0.2 \mathrm{mmol}, 0.2$ equiv.), $\mathrm{CuBr}_{2}$ (22.3 mg, $0.1 \mathrm{mmol}, 0.1$ equiv.), and hexafluoropropanol $(0.12 \mathrm{mmol} / \mathrm{mL})$. The purification of the dry crude performed by column chromatography on silica gel provides the amide $5 \mathrm{w}$ ( $125 \mathrm{mg}, 0.64 \mathrm{mmol}, 64 \%$ ).

Alternatively, the title compound was prepared starting with an appropriate 2-bromo2,2-difluoroacetamide $1 \mathrm{r}(214 \mathrm{mg}$, $1.0 \mathrm{mmol}, 1.0$ equiv.), $\mathrm{KF}$ (116 mg, $2.0 \mathrm{mmol}, 2.0$ equiv.), $\mathrm{MgCl}_{2}$ (95 mg, $1.0 \mathrm{mmol}, 1.0$ equiv.), aryl sulphonium salt $4 \mathbf{d}(516 \mathrm{mg}, 1.6 \mathrm{mmol}, 1.6$ equiv.), the $\mathbf{L} 2$ (202 $\mathrm{mg}, 0.25 \mathrm{mmol}, 0.25$ equiv.), [ $\mathrm{Ru}\left(\mathrm{p} \text {-cymene) } \mathrm{Cl}_{2}\right]_{2}$ (122 $\mathrm{mg}, 0.2 \mathrm{mmol}, 0.2$ equiv.), $\mathrm{CuBr}_{2}(67 \mathrm{mg}, 0.3 \mathrm{mmol}, 0.3$ equiv.), and hexafluoropropanol $(0.12 \mathrm{mmol} / \mathrm{mL})$. The purification of the dry crude performed by column chromatography on silica gel provides the amide $5 \mathrm{w}$ (113 $\mathrm{mg}, 0.58 \mathrm{mmol}, 58 \%)$.

Flash column chromatography was performed using a mixture of hexane/ethyl acetate 7:1 as an eluent to provide the corresponding amide product.

Colorless solid, mp 142-143 ${ }^{\circ} \mathrm{C} .{ }^{1} \mathrm{H}-\mathrm{NMR}\left(500 \mathrm{MHz}, \mathrm{CDCl}_{3}\right): \delta 0.83-0.86\left(\mathrm{~m}, 2 \mathrm{H}, \mathrm{CH}_{2}\right)$, $1.06-1.09\left(\mathrm{~m}, 2 \mathrm{H}, \mathrm{CH}_{2}\right), 1.48-1.53(\mathrm{~m}, 1 \mathrm{H}, \mathrm{CH}), 7.04\left(\mathrm{~d}, 1 \mathrm{H},{ }^{3} \mathrm{~J}=7.9 \mathrm{~Hz}, \mathrm{CH}_{\mathrm{Ar}}\right), 7.20(\mathrm{t}, 1 \mathrm{H}$, $\left.{ }^{3} J=8.7 \mathrm{~Hz}, \mathrm{CH}_{\mathrm{Ar}}\right), 7.32\left(\mathrm{~d}, 1 \mathrm{H},{ }^{3} \mathrm{~J}=7.9 \mathrm{~Hz}, \mathrm{CH}_{\mathrm{Ar}}\right), 7.63$ (br. s, $\left.1 \mathrm{H}, \mathrm{CH}_{\mathrm{Ar}}\right), 7.74(\mathrm{~s}, 1 \mathrm{H}, \mathrm{NH})$.

$\left.{ }^{13} \mathrm{C}_{1}{ }^{1} \mathrm{H}\right\}$-NMR $\left(126 \mathrm{MHz}, \mathrm{CDCl}_{3}\right): \delta 8.2,15.7,17.7,19.9,124.0,129.9,134.6,139.2,172.3$. HRMS (TOF MS ES+): Calcd for $\mathrm{C}_{10} \mathrm{H}_{11} \mathrm{NOCl}(\mathrm{M}+\mathrm{H})$ 196.0532. Found 196.0529.

[1, $1^{\prime}$-biphenyl]-4-yl(pyrrolidin-1-yl)methanone $5 x$. The title compound was prepared starting with an appropriate 2-bromo-2,2-difluoroacetamide $1 \mathrm{~s}$ (228 mg, $1.0 \mathrm{mmol}, 1.0$ equiv.), $\mathrm{KF}$ (116 mg, $2.0 \mathrm{mmol}, 2.0$ equiv.), $\mathrm{MgCl}_{2}$ (95 mg, $1.0 \mathrm{mmol}, 1.0$ equiv.), appropriate aryl boronic acid $2 \mathbf{e}\left(257 \mathrm{mg}, 1.3 \mathrm{mmol}, 1.3\right.$ equiv.), the $\mathbf{~} \mathbf{1}$ ( $130 \mathrm{mg}, 0.2 \mathrm{mmol}, 0.2$ equiv.), $\mathrm{CuBr}_{2}$ (22.3 $\mathrm{mg}, 0.1 \mathrm{mmol}, 0.1$ equiv.), and hexafluoropropanol $(0.12 \mathrm{mmol} / \mathrm{mL})$. The purification 
of the dry crude performed by column chromatography on silica gel provides the amide $5 \mathbf{x}$ (326 mg, $0.91 \mathrm{mmol}, 91 \%$ ).

Alternatively, the title compound was prepared starting with an appropriate 2-bromo2,2-difluoroacetamide $1 \mathrm{~s} \mathrm{(228} \mathrm{mg,} 1.0 \mathrm{mmol}, 1.0$ equiv.), $\mathrm{KF}$ (116 mg, $2.0 \mathrm{mmol}, 2.0$ equiv.), $\mathrm{MgCl}_{2}$ (95 mg, $1.0 \mathrm{mmol}, 1.0$ equiv.), appropriate trialkoxysilane $3 \mathrm{e}(442 \mathrm{mg}, 1.4 \mathrm{mmol}$, 1.4 equiv.), the $\mathbf{L 1}$ (130 mg, $0.2 \mathrm{mmol}, 0.2$ equiv.), $\mathrm{CuBr}_{2}$ ( $22.3 \mathrm{mg}, 0.1 \mathrm{mmol}, 0.1$ equiv.), and hexafluoropropanol $(0.12 \mathrm{mmol} / \mathrm{mL})$. The purification of the dry crude performed by column chromatography on silica gel provides the amide $5 x(223 \mathrm{mg}, 0.89 \mathrm{mmol}, 89 \%)$.

Alternatively, the title compound was prepared starting with an appropriate 2-bromo2,2-difluoroacetamide $1 \mathrm{~s}$ ( $228 \mathrm{mg}$, $1.0 \mathrm{mmol}, 1.0$ equiv.), $\mathrm{KF}$ (116 mg, $2.0 \mathrm{mmol}, 2.0$ equiv.), $\mathrm{MgCl}_{2}$ (95 mg, $1.0 \mathrm{mmol}, 1.0$ equiv.), aryl sulphonium salt $4 \mathrm{e}$ (582 mg, $1.6 \mathrm{mmol}, 1.6$ equiv.), the $\mathbf{L} 2$ ( $202 \mathrm{mg}, 0.25 \mathrm{mmol}, 0.25$ equiv.), [ $\mathrm{Ru}\left(\mathrm{p} \text {-cymene) } \mathrm{Cl}_{2}\right]_{2}$ ( $122 \mathrm{mg}, 0.2 \mathrm{mmol}, 0.2$ equiv.), $\mathrm{CuBr}_{2}(67 \mathrm{mg}, 0.3 \mathrm{mmol}, 0.3$ equiv.), and hexafluoropropanol $(0.12 \mathrm{mmol} / \mathrm{mL})$. The purification of the dry crude performed by column chromatography on silica gel provides the amide $5 x$ (206 mg, $0.82 \mathrm{mmol}, 82 \%$ ).

Flash column chromatography was performed using a mixture of hexane/ethyl acetate 8:1 as an eluent to provide the corresponding amide product.

White solid, mp 139-140 ${ }^{\circ} \mathrm{C} .{ }^{1} \mathbf{H}-\mathbf{N M R}\left(500 \mathrm{MHz}, \mathrm{CDCl}_{3}\right): \delta 1.86$ (m, 4H, Pyrr), 3.39 (s, $2 \mathrm{H}$, Pyrr), $3.58\left(\mathrm{~s}, 2 \mathrm{H}\right.$, Pyrr), $7.34\left(\mathrm{t}, 1 \mathrm{H},{ }^{3} \mathrm{~J}=7.7 \mathrm{~Hz}, \mathrm{CH}_{\mathrm{Ar}}\right), 7.43\left(\mathrm{t}, 2 \mathrm{H},{ }^{3} \mathrm{~J}=7.7 \mathrm{~Hz}, \mathrm{CH}_{\mathrm{Ar}}\right)$, $7.57-7.59\left(\mathrm{~m}, 6 \mathrm{H}, \mathrm{CH}_{\mathrm{Ar}}\right)$.

$\left.{ }^{13} \mathrm{C}^{1}{ }^{1} \mathrm{H}\right\}-\mathrm{NMR}\left(126 \mathrm{MHz}, \mathrm{CDCl}_{3}\right): \delta 24.3,26.3,46.1,49.5,126.7,127.0,127.6,128.7$, $135.8,140.1,142.4,169.3$.

HRMS (TOF MS ES+): Calcd for $\mathrm{C}_{17} \mathrm{H}_{18} \mathrm{NO}(\mathrm{M}+\mathrm{H})$ 252.1391. Found 252.1388.

N-phenethyl-1-naphthamide 5y. The title compound was prepared starting with an appropriate 2-bromo-2,2-difluoroacetamide $1 \mathrm{t}(278 \mathrm{mg}$, $1.0 \mathrm{mmol}$, 1.0 equiv.), KF (116 mg, $2.0 \mathrm{mmol}, 2.0$ equiv.), $\mathrm{MgCl}_{2}$ (95 mg, $1.0 \mathrm{mmol}, 1.0$ equiv.), appropriate aryl boronic acid 2d (224 mg, $1.3 \mathrm{mmol}, 1.3$ equiv.), the $\mathbf{~} \mathbf{1}$ (130 mg, $0.2 \mathrm{mmol}, 0.2$ equiv.), $\mathrm{CuBr}_{2}$ (22.3 mg, $0.1 \mathrm{mmol}, 0.1$ equiv.), and hexafluoropropanol $(0.12 \mathrm{mmol} / \mathrm{mL})$. The purification of the dry crude performed by column chromatography on silica gel provides the amide $5 \mathrm{y}$ ( $228 \mathrm{mg}$, $0.83 \mathrm{mmol}, 83 \%$ ). The gram scale synthesis was performed on $10 \mathrm{mmol}$ of the starting 2-bromo-2,2-difluoroacetamide $\mathbf{1 t}$ and the amide $5 \mathrm{y}$ was prepared in $78 \%$ yield $(2.15 \mathrm{~g}$, $7.8 \mathrm{mmol})$.

Alternatively, the title compound was prepared starting with an appropriate 2-bromo2,2-difluoroacetamide $1 \mathrm{t}(278 \mathrm{mg}, 1.0 \mathrm{mmol}, 1.0$ equiv.), $\mathrm{KF}$ (116 mg, $2.0 \mathrm{mmol}, 2.0$ equiv.), $\mathrm{MgCl}_{2}$ (95 mg, $1.0 \mathrm{mmol}, 1.0$ equiv.), appropriate trialkoxysilane $3 \mathrm{~d}(347 \mathrm{mg}, 1.4 \mathrm{mmol}$, 1.4 equiv.), the $\mathbf{L 1}\left(130 \mathrm{mg}, 0.2 \mathrm{mmol}, 0.2\right.$ equiv.), $\mathrm{CuBr}_{2}$ ( $22.3 \mathrm{mg}, 0.1 \mathrm{mmol}, 0.1$ equiv.), and hexafluoropropanol $(0.12 \mathrm{mmol} / \mathrm{mL})$. The purification of the dry crude performed by column chromatography on silica gel provides the amide $5 \mathrm{y}(212 \mathrm{mg}, 0.77 \mathrm{mmol}$, $77 \%$ ). The gram scale synthesis was performed on $10 \mathrm{mmol}$ of the starting 2-bromo-2,2difluoroacetamide $\mathbf{1 t}$ and the amide $5 \mathrm{y}$ was prepared in $70 \%$ yield $(1.93 \mathrm{~g}, 7 \mathrm{mmol})$.

Flash column chromatography was performed using a mixture of hexane/ethyl acetate 5:1 as an eluent to provide the corresponding amide product.

White solid, mp 117-118 ${ }^{\circ} \mathrm{C} .{ }^{1} \mathrm{H}-\mathrm{NMR}\left(500 \mathrm{MHz}, \mathrm{CDCl}_{3}\right): \delta 3.00\left(\mathrm{t}, 2 \mathrm{H},{ }^{3} \mathrm{~J}=6.1 \mathrm{~Hz}\right.$, $\left.\mathrm{CH}_{2}\right), 3.80\left(\mathrm{q}, 2 \mathrm{H},{ }^{3} \mathrm{~J}=7.2 \mathrm{~Hz}, \mathrm{CH}_{2}\right), 6.13(\mathrm{~s}, 1 \mathrm{H}, \mathrm{NH}), 7.27-7.28\left(\mathrm{~m}, 3 \mathrm{H}, \mathrm{CH}_{\mathrm{Ar}}\right), 7.33-7.37$ $\left(\mathrm{m}, 2 \mathrm{H}, \mathrm{CH}_{\mathrm{Ar}}\right), 7.40-7.43\left(\mathrm{~m}, 1 \mathrm{H}, \mathrm{CH}_{\mathrm{Ar}}\right), 7.48-7.54\left(\mathrm{~m}, 3 \mathrm{H}, \mathrm{CH}_{\mathrm{Ar}}\right), 7.86-7.88\left(\mathrm{~m}, 1 \mathrm{H}, \mathrm{CH}_{\mathrm{Ar}}\right)$, $7.89\left(\mathrm{~d}, 1 \mathrm{H},{ }^{3} \mathrm{~J}=8.0 \mathrm{~Hz}, \mathrm{CH}_{\mathrm{Ar}}\right), 8.20-8.22\left(\mathrm{~m}, 1 \mathrm{H}, \mathrm{CH}_{\mathrm{Ar}}\right)$.

${ }^{13} \mathrm{C}\left\{{ }^{1} \mathrm{H}\right\}-N M R\left(126 \mathrm{MHz}, \mathrm{CDCl}_{3}\right): \delta 35.6,41.0,124.6,124.85,125.3,126.3,126.5,127.0$, $128.2,128.7,128.8,130.0,130.4,133.6,134.5,138.7$.

HRMS (TOF MS ES+): Calcd for $\mathrm{C}_{19} \mathrm{H}_{18} \mathrm{NO}(\mathrm{M}+\mathrm{H})$ 276.1396. Found 276.1388.

$N$-benzyl-3-chlorobenzamide $5 z$. The title compound was prepared starting with an appropriate 2-bromo-2,2-difluoroacetamide $1 \mathbf{u}(264 \mathrm{mg}, 1.0 \mathrm{mmol}$, 1.0 equiv.), $\mathrm{KF}$ (116 mg, $2.0 \mathrm{mmol}, 2.0$ equiv.), $\mathrm{MgCl}_{2}$ (95 mg, $1.0 \mathrm{mmol}, 1.0$ equiv.), appropriate aryl boronic acid 2k (203 mg, $1.3 \mathrm{mmol}, 1.3$ equiv.), the $\mathbf{L 1}$ (130 mg, $0.2 \mathrm{mmol}, 0.2$ equiv.), $\mathrm{CuBr}_{2}$ (22.3 mg, $0.1 \mathrm{mmol}, 0.1$ equiv.), and hexafluoropropanol $(0.12 \mathrm{mmol} / \mathrm{mL})$. The purification of the dry 
crude performed by column chromatography on silica gel provides the amide $5 \mathbf{z}$ (209 mg, $0.85 \mathrm{mmol}, 85 \%$ ).

Alternatively, the title compound was prepared starting with an appropriate 2-bromo2,2-difluoroacetamide $1 \mathbf{u}$ (264 mg, $1.0 \mathrm{mmol}, 1.0$ equiv.), $\mathrm{KF}$ (116 mg, $2.0 \mathrm{mmol}, 2.0$ equiv.), $\mathrm{MgCl}_{2}$ (95 mg, $1.0 \mathrm{mmol}, 1.0$ equiv.), appropriate trialkoxysilane $3 \mathbf{i}(372 \mathrm{mg}, 1.4 \mathrm{mmol}$, 1.4 equiv.), the $\mathbf{L 1}$ (130 mg, $0.2 \mathrm{mmol}, 0.2$ equiv.), $\mathrm{CuBr}_{2}$ (22.3 mg, $0.1 \mathrm{mmol}, 0.1$ equiv.), and hexafluoropropanol $(0.12 \mathrm{mmol} / \mathrm{mL})$. The purification of the dry crude performed by column chromatography on silica gel provides the amide $5 z$ (194 mg, $0.79 \mathrm{mmol}, 79 \%$ ).

Flash column chromatography was performed using a mixture of hexane/ethyl acetate 2:1 as an eluent to provide the corresponding amide product.

Yellowish solid, mp 93-94 ${ }^{\circ} \mathrm{C} .{ }^{1} \mathbf{H}-N M R\left(500 \mathrm{MHz}, \mathrm{CDCl}_{3}\right): \delta 4.57\left(\mathrm{~d}, 2 \mathrm{H},{ }^{3} \mathrm{~J}=5.5 \mathrm{~Hz}\right.$, $\left.\mathrm{CH}_{2}\right), 6.77(\mathrm{~s}, 1 \mathrm{H}, \mathrm{NH}), 7.27-7.35\left(\mathrm{~m}, 6 \mathrm{H}, \mathrm{CH}_{\mathrm{Ar}}\right), 7.44\left(\mathrm{dd}, 1 \mathrm{H},{ }^{3} \mathrm{~J}=8.0 \mathrm{~Hz},{ }^{4} J=1.0 \mathrm{~Hz}\right.$, $\left.\mathrm{CH}_{\mathrm{Ar}}\right), 7.64\left(\mathrm{~d}, 1 \mathrm{H},{ }^{3} \mathrm{~J}=8.0 \mathrm{~Hz}, \mathrm{CH}_{\mathrm{Ar}}\right), 7.77\left(\mathrm{~d}, 1 \mathrm{H},{ }^{4} \mathrm{~J}=1.8 \mathrm{~Hz}, \mathrm{CH}_{\mathrm{Ar}}\right)$.

$\left.{ }^{13} \mathrm{C}_{1}{ }^{1} \mathrm{H}\right\}-N M R\left(126 \mathrm{MHz}, \mathrm{CDCl}_{3}\right): \delta 44.1,125.1,127.3,127.6,127.8,128.7,129.8,131.5$, $134.7,136.1,137.8,166.1$.

HRMS (TOF MS ES+): Calcd for $\mathrm{C}_{14} \mathrm{H}_{13} \mathrm{NOCl}(\mathrm{M}+\mathrm{H})$ 246.0686. Found 246.0686.

$\mathrm{N}$-(4-fluorobenzyl)-2-methylbenzamide 5aa. The title compound was prepared starting with an appropriate 2-bromo-2,2-difluoroacetamide $1 \mathbf{v}(282 \mathrm{mg}, 1.0 \mathrm{mmol}, 1.0$ equiv.), $\mathrm{KF}$ (116 mg, $2.0 \mathrm{mmol}, 2.0$ equiv.), $\mathrm{MgCl}_{2}$ ( $95 \mathrm{mg}, 1.0 \mathrm{mmol}, 1.0$ equiv.), appropriate aryl boronic acid 21 ( $203 \mathrm{mg}, 1.3 \mathrm{mmol}, 1.3$ equiv.), the $\mathbf{L 1}$ ( $130 \mathrm{mg}, 0.2 \mathrm{mmol}, 0.2$ equiv.), $\mathrm{CuBr}_{2}$ (22.3 $\mathrm{mg}, 0.1 \mathrm{mmol}, 0.1$ equiv.), and hexafluoropropanol $(0.12 \mathrm{mmol} / \mathrm{mL})$. The purification of the dry crude performed by column chromatography on silica gel provides the amide 5aa (189 mg, $0.72 \mathrm{mmol}, 72 \%)$.

Alternatively, the title compound was prepared starting with an appropriate 2-bromo2,2-difluoroacetamide 1v (282 mg, $1.0 \mathrm{mmol}, 1.0$ equiv.), $\mathrm{KF}$ (116 mg, $2.0 \mathrm{mmol}, 2.0$ equiv.), $\mathrm{MgCl}_{2}$ (95 mg, $1.0 \mathrm{mmol}, 1.0$ equiv.), aryl sulphonium salt $4 \mathrm{f}(516 \mathrm{mg}, 1.6 \mathrm{mmol}, 1.6$ equiv.), the $\mathbf{L} 2$ (202 mg, $0.25 \mathrm{mmol}, 0.25$ equiv.), [ $\mathrm{Ru}\left(\mathrm{p} \text {-cymene) } \mathrm{Cl}_{2}\right]_{2}$ ( $122 \mathrm{mg}, 0.2 \mathrm{mmol}, 0.2$ equiv.), $\mathrm{CuBr}_{2}$ (67 mg, $0.3 \mathrm{mmol}, 0.3$ equiv.), and hexafluoropropanol $(0.12 \mathrm{mmol} / \mathrm{mL})$. The purification of the dry crude performed by column chromatography on silica gel provides the amide 5 aa (158 mg, $0.60 \mathrm{mmol}, 60 \%)$.

Alternatively, the title compound was prepared starting with an appropriate 2-bromo2,2-difluoroacetamide 1v (282 mg, $1.0 \mathrm{mmol}, 1.0$ equiv.), KF (116 mg, $2.0 \mathrm{mmol}, 2.0$ equiv.), $\mathrm{MgCl}_{2}$ (95 mg, $1.0 \mathrm{mmol}, 1.0$ equiv.), appropriate potassium (2-chlorophenyl)trifluoroborate ( $283 \mathrm{mg}, 1.3 \mathrm{mmol}, 1.3$ equiv.), the $\mathbf{L 1}$ (130 mg, $0.2 \mathrm{mmol}, 0.2$ equiv.), $\mathrm{CuBr}_{2}(22.3 \mathrm{mg}, 0.1$ $\mathrm{mmol}, 0.1$ equiv.), and hexafluoropropanol $(0.12 \mathrm{mmol} / \mathrm{mL})$. The purification of the dry crude performed by column chromatography on silica gel provides the amide $5 \mathbf{a a}(181 \mathrm{mg}$, $0.69 \mathrm{mmol}, 69 \%)$.

Flash column chromatography was performed using a mixture of hexane/ethyl acetate 4:1 as an eluent to provide the corresponding amide product.

Colorless solid, mp 119-120 ${ }^{\circ} \mathrm{C} .{ }^{1} \mathrm{H}-\mathrm{NMR}\left(500 \mathrm{MHz}, \mathrm{CDCl}_{3}\right): \delta 2.36(\mathrm{~s}, 3 \mathrm{H}, \mathrm{Me}), 4.46$ $\left(\mathrm{d}, 2 \mathrm{H},{ }^{3} \mathrm{~J}=5.7 \mathrm{~Hz}, \mathrm{CH}_{2}\right), 6.40(\mathrm{~s}, 1 \mathrm{H}, \mathrm{NH}), 6.97\left(\mathrm{t}, 2 \mathrm{H},{ }^{3} \mathrm{~J}=8.7 \mathrm{~Hz}, \mathrm{CH}_{\mathrm{Ar}}\right), 7.11-7.17(\mathrm{~m}, 2 \mathrm{H}$, $\left.\mathrm{CH}_{\mathrm{Ar}}\right), 7.23-7.28\left(\mathrm{~m}, 4 \mathrm{H}, \mathrm{CH}_{\mathrm{Ar}}\right)$.

$\left.{ }^{13} \mathrm{C}^{1}{ }^{1} \mathrm{H}\right\}$-NMR $\left(126 \mathrm{MHz}, \mathrm{CDCl}_{3}\right): \delta 19.7,42.9,115.4\left(\mathrm{~d}, J_{\mathrm{CF}}=22.0 \mathrm{~Hz}\right), 125.6,126.6$, $129.3\left(\mathrm{~d}, J_{C F}=8.9 \mathrm{~Hz}\right), 129.9,130.9,134.1\left(\mathrm{~d}, J_{C F}=2.3 \mathrm{~Hz}\right), 136.0\left(\mathrm{~d}, J_{C F}=2.7 \mathrm{~Hz}\right), 126.1(\mathrm{~d}$, $\left.{ }^{1} J_{C F}=243.3 \mathrm{~Hz}\right), 169.9$.

HRMS (TOF MS ES+): Calcd for $\mathrm{C}_{15} \mathrm{H}_{15} \mathrm{NOF}(\mathrm{M}+\mathrm{H})$ 244.1143. Found 244.1138.

$\mathrm{N}, \mathrm{N}$-dibenzyl-4-fluorobenzamide 5ab. The title compound was prepared starting with an appropriate 2-bromo-2,2-difluoroacetamide $1 \mathrm{~m}$ (354 mg, $1.0 \mathrm{mmol}, 1.0$ equiv.), $\mathrm{KF}$ (116 mg, $2.0 \mathrm{mmol}, 2.0$ equiv.), $\mathrm{MgCl}_{2}$ (95 mg, $1.0 \mathrm{mmol}, 1.0$ equiv.), appropriate aryl boronic acid $\mathbf{2 f}$ ( $174 \mathrm{mg}, 1.3 \mathrm{mmol}, 1.3$ equiv.), the $\mathbf{L 1}$ ( $130 \mathrm{mg}, 0.2 \mathrm{mmol}, 0.2$ equiv.), $\mathrm{CuBr}_{2}$ (22.3 $\mathrm{mg}, 0.1 \mathrm{mmol}, 0.1$ equiv.), and hexafluoropropanol $(0.12 \mathrm{mmol} / \mathrm{mL})$. The purification of the dry crude performed by column chromatography on silica gel provides the amide 5 ab $(290 \mathrm{mg}, 0.91 \mathrm{mmol}, 91 \%)$. The gram scale synthesis was performed on $10 \mathrm{mmol}$ of the 
starting 2-bromo-2,2-difluoroacetamide $\mathbf{1 m}$ and the amide $5 \mathrm{ab}$ was prepared in $83 \%$ yield $(2.65 \mathrm{~g}, 8.3 \mathrm{mmol})$.

Alternatively, the title compound was prepared starting with an appropriate 2-bromo2,2-difluoroacetamide $1 \mathrm{~m}$ ( $354 \mathrm{mg}, 1.0 \mathrm{mmol}, 1.0$ equiv.), KF (116 mg, $2.0 \mathrm{mmol}, 2.0$ equiv.), $\mathrm{MgCl}_{2}$ (95 mg, $1.0 \mathrm{mmol}, 1.0$ equiv.), appropriate trialkoxysilane $3 \mathrm{f}(361 \mathrm{mg}, 1.4 \mathrm{mmol}$, 1.4 equiv.), the $\mathbf{L 1}$ (130 mg, $0.2 \mathrm{mmol}, 0.2$ equiv.), $\mathrm{CuBr}_{2}$ ( $22.3 \mathrm{mg}, 0.1 \mathrm{mmol}, 0.1$ equiv.), and hexafluoropropanol $(0.12 \mathrm{mmol} / \mathrm{mL})$. The purification of the dry crude performed by column chromatography on silica gel provides the amide $5 \mathrm{ab}(287 \mathrm{mg}, 0.90 \mathrm{mmol}$, $90 \%$ ). The gram scale synthesis was performed on $10 \mathrm{mmol}$ of the starting 2-bromo-2,2difluoroacetamide $1 \mathrm{~m}$ and the amide $\mathbf{5 a b}$ was prepared in $78 \%$ yield $(2.45 \mathrm{~g}, 7.8 \mathrm{mmol})$.

Alternatively, the title compound was prepared starting with an appropriate 2-bromo2,2-difluoroacetamide $1 \mathrm{~m}$ ( $354 \mathrm{mg}, 1.0 \mathrm{mmol}, 1.0$ equiv.), KF (116 mg, $2.0 \mathrm{mmol}, 2.0$ equiv.), $\mathrm{MgCl}_{2}$ (95 mg, $1.0 \mathrm{mmol}, 1.0$ equiv.), aryl sulphonium salt $4 \mathrm{~g}$ ( $490 \mathrm{mg}, 1.6 \mathrm{mmol}, 1.6$ equiv.), the $\mathbf{L} 2$ (202 mg, $0.25 \mathrm{mmol}, 0.25$ equiv.), [ $\mathrm{Ru}\left(\mathrm{p} \text {-cymene) } \mathrm{Cl}_{2}\right]_{2}$ (122 mg, $0.2 \mathrm{mmol}, 0.2$ equiv.), $\mathrm{CuBr}_{2}(67 \mathrm{mg}, 0.3 \mathrm{mmol}, 0.3$ equiv.), and hexafluoropropanol $(0.12 \mathrm{mmol} / \mathrm{mL})$. The purification of the dry crude performed by column chromatography on silica gel provides the amide $5 \mathrm{ab}(281 \mathrm{mg}, 0.88 \mathrm{mmol}, 88 \%)$.

Alternatively, the title compound was prepared starting with an appropriate 2-bromo2,2-difluoroacetamide $1 \mathrm{~m}$ (354 mg, $1.0 \mathrm{mmol}, 1.0$ equiv.), KF (116 mg, $2.0 \mathrm{mmol}, 2.0$ equiv.), $\mathrm{MgCl}_{2}$ (95 mg, $1.0 \mathrm{mmol}, 1.0$ equiv.), appropriate 2-(4-fluorophenyl)-4,4,5,5-tetramethyl1,3,2-dioxaborolane ( $289 \mathrm{mg}, 1.3 \mathrm{mmol}, 1.3$ equiv.), the $\mathbf{L 1}$ ( $130 \mathrm{mg}, 0.2 \mathrm{mmol}, 0.2$ equiv.), $\mathrm{CuBr}_{2}$ (22.3 mg, $0.1 \mathrm{mmol}, 0.1$ equiv.), and hexafluoropropanol $(0.12 \mathrm{mmol} / \mathrm{mL})$. The purification of the dry crude performed by column chromatography on silica gel provides the amide $5 \mathrm{ab}$ ( $284 \mathrm{mg}, 0.89 \mathrm{mmol}, 89 \%)$.

Flash column chromatography was performed using a mixture of hexane/ethyl acetate 3:1 as an eluent to provide the corresponding amide product.

White solid, mp 86-87 ${ }^{\circ} \mathrm{C} .{ }^{1} \mathrm{H}-\mathrm{NMR}\left(500 \mathrm{MHz}, \mathrm{CDCl}_{3}\right): \delta 4.20\left(\mathrm{~s}, 2 \mathrm{H}, \mathrm{CH}_{2}\right), 4.71(\mathrm{~s}$, $\left.2 \mathrm{H}, \mathrm{CH}_{2}\right), 7.07\left(\mathrm{t}, 2 \mathrm{H},{ }^{3} \mathrm{~J}=7.6 \mathrm{~Hz}, \mathrm{CH}_{\mathrm{Ar}}\right), 7.15$ (br. s, $\left.2 \mathrm{H}, \mathrm{CH}_{\mathrm{Ar}}\right), 7.30-7.33\left(\mathrm{~m}, 4 \mathrm{H}, \mathrm{CH}_{\mathrm{Ar}}\right)$, 7.36-7.39 (m, 4H, $\left.\mathrm{CH}_{\mathrm{Ar}}\right), 8.50-7.53\left(\mathrm{~m}, 2 \mathrm{H}, \mathrm{CH}_{\mathrm{Ar}}\right)$.

$\left.{ }^{13} \mathrm{C}_{1}{ }^{1} \mathrm{H}\right\}$-NMR $\left(126 \mathrm{MHz}, \mathrm{CDCl}_{3}\right): \delta 47.2,51.6,115.6\left(\mathrm{~d}, J_{\mathrm{CF}}=22.2 \mathrm{~Hz}\right), 126.8(\mathrm{~m})$, $127.7(\mathrm{~m}), 128.4(\mathrm{~m}), 128.7(\mathrm{~m}), 128.9,129.0,132.0(\mathrm{~m}), 136.5\left(\mathrm{~d}, J_{C F}=67.9 \mathrm{~Hz}\right), 163.3(\mathrm{~d}$, $\left.{ }^{1} J_{C F}=247.5 \mathrm{~Hz}\right), 171.3$.

HRMS (TOF MS ES+): Calcd for $\mathrm{C}_{21} \mathrm{H}_{19} \mathrm{NOF}(\mathrm{M}+\mathrm{H})$ 320.1455. Found 320.1451.

$\mathrm{N}, \mathrm{N}$-dibenzyl-4-((trifluoromethyl)thio)benzamide 5ac. The title compound was prepared starting with an appropriate 2-bromo-2,2-difluoroacetamide $1 \mathrm{~m}$ ( $354 \mathrm{mg}, 1.0 \mathrm{mmol}$, 1.0 equiv.), KF (116 mg, $2.0 \mathrm{mmol}, 2.0$ equiv.), $\mathrm{MgCl}_{2}$ (95 mg, $1.0 \mathrm{mmol}, 1.0$ equiv.), appropriate aryl boronic acid $2 \mathrm{~s}(289 \mathrm{mg}, 1.3 \mathrm{mmol}, 1.3$ equiv.), the $\mathbf{L 1}(130 \mathrm{mg}, 0.2 \mathrm{mmol}$, 0.2 equiv.), $\mathrm{CuBr}_{2}(22.3 \mathrm{mg}, 0.1 \mathrm{mmol}, 0.1$ equiv.), and hexafluoropropanol $(0.12 \mathrm{mmol} / \mathrm{mL})$. The purification of the dry crude performed by column chromatography on silica gel provides the amide $5 \mathrm{ac}(337 \mathrm{mg}, 0.84 \mathrm{mmol}, 84 \%)$.

Flash column chromatography was performed using a mixture of hexane/ethyl acetate 3:1 as an eluent to provide the corresponding amide product.

Colorless solid, mp 68-70 ${ }^{\circ} \mathrm{C} .{ }^{1} \mathrm{H}-\mathrm{NMR}\left(500 \mathrm{MHz}, \mathrm{CDCl}_{3}\right): \delta 4.39\left(\mathrm{~s}, 2 \mathrm{H}, \mathrm{CH}_{2}\right), 4.74$ $\left(\mathrm{s}, 2 \mathrm{H}, \mathrm{CH}_{2}\right), 7.13\left(\mathrm{~d}, 2 \mathrm{H},{ }^{3} \mathrm{~J}=6.9 \mathrm{~Hz}, \mathrm{CH}_{\mathrm{Ar}}\right), 7.30-7.34\left(\mathrm{~m}, 4 \mathrm{H}, \mathrm{CH}_{\mathrm{Ar}}\right), 7.37-7.40(\mathrm{~m}, 4 \mathrm{H}$, $\left.\mathrm{CH}_{\mathrm{Ar}}\right), 7.54\left(\mathrm{~d}, 2 \mathrm{H},{ }^{3} \mathrm{~J}=8.1 \mathrm{~Hz}, \mathrm{CH}_{\mathrm{Ar}}\right), 7.67\left(\mathrm{~d}, 2 \mathrm{H},{ }^{3} \mathrm{~J}=8.1 \mathrm{~Hz}, \mathrm{CH}_{\mathrm{Ar}}\right)$.

$\left.{ }^{13} \mathrm{C}_{1}{ }^{1} \mathrm{H}\right\}-\mathrm{NMR}\left(126 \mathrm{MHz}, \mathrm{CDCl}_{3}\right): \delta 47.1,51.5,127.1\left(\mathrm{q},{ }^{1} J_{\mathrm{CF}}=258.2 \mathrm{~Hz}, \mathrm{SCF}_{3}\right), 126.9$, $127.7,128.2,128.4,128.8,129.0,135.9,136.2,136.5,138.6,170.8$.

HRMS (TOF MS ES+): Calcd for $\mathrm{C}_{22} \mathrm{H}_{19} \mathrm{NOF}_{3} \mathrm{~S}(\mathrm{M}+\mathrm{H})$ 402.1141. Found 402.1139.

$\mathrm{N}, \mathrm{N}$-dibenzyl-2-(trifluoromethyl)benzamide 5ad. The title compound was prepared starting with an appropriate 2-bromo-2,2-difluoroacetamide $1 \mathrm{~m} \mathrm{(354} \mathrm{mg,} 1.0 \mathrm{mmol}$, 1.0 equiv.), $\mathrm{KF}$ (116 mg, $2.0 \mathrm{mmol}, 2.0$ equiv.), $\mathrm{MgCl}_{2}$ (95 mg, $1.0 \mathrm{mmol}, 1.0$ equiv.), appropriate aryl boronic acid $2 \mathrm{o}(247 \mathrm{mg}, 1.3 \mathrm{mmol}, 1.3$ equiv.), the $\mathbf{L 1}(130 \mathrm{mg}, 0.2 \mathrm{mmol}$, 0.2 equiv.), $\mathrm{CuBr}_{2}$ (22.3 mg, $0.1 \mathrm{mmol}, 0.1$ equiv.), and hexafluoropropanol $(0.12 \mathrm{mmol} / \mathrm{mL})$. 
The purification of the dry crude performed by column chromatography on silica gel provides the amide $5 \mathrm{ac}(247 \mathrm{mg}, 0.67 \mathrm{mmol}, 67 \%)$.

Alternatively, the title compound was prepared starting with an appropriate 2-bromo2,2-difluoroacetamide $1 \mathrm{~m}$ ( $354 \mathrm{mg}, 1.0 \mathrm{mmol}, 1.0$ equiv.), KF (116 mg, $2.0 \mathrm{mmol}, 2.0$ equiv.), $\mathrm{MgCl}_{2}$ (95 mg, $1.0 \mathrm{mmol}, 1.0$ equiv.), appropriate trialkoxysilane 31 (372 mg, $1.4 \mathrm{mmol}$, 1.4 equiv.), the $\mathbf{L 1}$ (130 mg, $0.2 \mathrm{mmol}, 0.2$ equiv.), $\mathrm{CuBr}_{2}$ (22.3 mg, $0.1 \mathrm{mmol}, 0.1$ equiv.), and hexafluoropropanol $(0.12 \mathrm{mmol} / \mathrm{mL})$. The purification of the dry crude performed by column chromatography on silica gel provides the amide $5 \mathrm{ab}(229 \mathrm{mg}, 0.62 \mathrm{mmol}, 62 \%$ ).

Flash column chromatography was performed using a mixture of hexane/ethyl acetate 4:1 as an eluent to provide the corresponding amide product.

Colorless solid, mp 135-136 ${ }^{\circ} \mathrm{C} .{ }^{1} \mathrm{H}-\mathrm{NMR}\left(500 \mathrm{MHz}, \mathrm{CDCl}_{3}\right): \delta 4.11\left(\mathrm{t}, 2 \mathrm{H},{ }^{3} \mathrm{~J}=15.3 \mathrm{~Hz}\right.$, $\left.\mathrm{CH}_{2}\right), 4.25\left(\mathrm{~d}, 1 \mathrm{H},{ }^{3} \mathrm{~J}=15.8 \mathrm{~Hz}, \mathrm{CH}_{2}\right), 5.33\left(\mathrm{~d}, 1 \mathrm{H},{ }^{3} \mathrm{~J}=14.8 \mathrm{~Hz}, \mathrm{CH}_{2}\right), 7.11\left(\mathrm{~m}, 2 \mathrm{H},{ }^{3} \mathrm{~J}=7.1 \mathrm{~Hz}\right.$, $\left.\mathrm{CH}_{\mathrm{Ar}}\right), 7.29-7.37\left(\mathrm{~m}, 8 \mathrm{H}, \mathrm{CH}_{\mathrm{Ar}}\right), 7.47-7.51\left(\mathrm{~m}, 2 \mathrm{H}, \mathrm{CH}_{\mathrm{Ar}}\right), 7.56\left(\mathrm{t}, 1 \mathrm{H},{ }^{3} \mathrm{~J}=7.1 \mathrm{~Hz}, \mathrm{CH}_{\mathrm{Ar}}\right)$, $7.71\left(\mathrm{~d}, 1 \mathrm{H},{ }^{3} \mathrm{~J}=7.8 \mathrm{~Hz}, \mathrm{CH}_{\mathrm{Ar}}\right)$.

$\left.{ }^{13} \mathrm{C}^{1}{ }^{1} \mathrm{H}\right\}$-NMR $\left(126 \mathrm{MHz}, \mathrm{CDCl}_{3}\right): \delta 46.4,51.1,123.7\left(\mathrm{q},{ }^{1} J_{C F}=274.8 \mathrm{~Hz}, \mathrm{CF}_{3}\right), 126.6$ (m), 127.3, 127.4, 127.7, 127.8, 128.5, 128.8, 129.1, 129.1, 132.1, 135.0, 135.5, 136.1, 169.2.

HRMS (TOF MS ES+): Calcd for $\mathrm{C}_{22} \mathrm{H}_{19} \mathrm{NOF}_{3}(\mathrm{M}+\mathrm{H})$ 370.1422. Found 370.1419.

N1,N1,N3,N3-tetrabenzylisophthalamide 5ae. The title compound was prepared starting with an appropriate 2-bromo-2,2-difluoroacetamide $1 \mathrm{~m}(885 \mathrm{mg}, 2.5 \mathrm{mmol}$, 2.5 equiv.), $\mathrm{KF}$ ( $232 \mathrm{mg}, 2.0 \mathrm{mmol}, 2.0$ equiv.), $\mathrm{MgCl}_{2}$ (190 mg, $2.0 \mathrm{mmol}, 2.0$ equiv.), appropriate aryl boronic acid $\mathbf{2 v}(166 \mathrm{mg}, 1.0 \mathrm{mmol}, 1.0$ equiv.), the $\mathbf{L 1}(260 \mathrm{mg}, 0.4 \mathrm{mmol}$, 0.4 equiv.), $\mathrm{CuBr}_{2}(44.6 \mathrm{mg}, 0.2 \mathrm{mmol}, 0.2$ equiv.), and hexafluoropropanol $(0.08 \mathrm{mmol} / \mathrm{mL})$. The purification of the dry crude performed by column chromatography on silica gel provides the amide $5 \mathrm{ae}(445 \mathrm{mg}, 0.85 \mathrm{mmol}, 85 \%)$.

Alternatively, the title compound was prepared starting with an appropriate 2-bromo2,2-difluoroacetamide $1 \mathrm{~m}$ ( $885 \mathrm{mg}, 2.5 \mathrm{mmol}, 2.5$ equiv.), KF (232 mg, $4.0 \mathrm{mmol}, 4.0$ equiv.), $\mathrm{MgCl}_{2}(190 \mathrm{mg}, 2.0 \mathrm{mmol}, 2.0$ equiv.), appropriate trialkoxysilane $3 \mathrm{q}(318 \mathrm{mg}, 1.0 \mathrm{mmol}$, 1.0 equiv.), the $\mathbf{L 1}$ ( $260 \mathrm{mg}, 0.4 \mathrm{mmol}, 0.4$ equiv.), $\mathrm{CuBr}_{2}$ ( $44.6 \mathrm{mg}, 0.2 \mathrm{mmol}, 0.2$ equiv.), and hexafluoropropanol $(0.08 \mathrm{mmol} / \mathrm{mL})$. The purification of the dry crude performed by column chromatography on silica gel provides the amide $5 \mathrm{ae}(419 \mathrm{mg}, 0.80 \mathrm{mmol}, 80 \%)$.

Flash column chromatography was performed using a mixture of hexane/ethyl acetate $1: 2$ as an eluent to provide the corresponding amide product.

White solid, mp 172-173 ${ }^{\circ} \mathrm{C} .{ }^{1} \mathrm{H}-\mathrm{NMR}\left(500 \mathrm{MHz}, \mathrm{CDCl}_{3}\right): \delta 4.39\left(\mathrm{~s}, 4 \mathrm{H}, 2 \mathrm{xCH}_{2}\right), 4.71$ $\left(\mathrm{s}, 4 \mathrm{H}, 2 \mathrm{CCH}_{2}\right), 7.12\left(\mathrm{~d}, 4 \mathrm{H},{ }^{3} \mathrm{~J}=6.8 \mathrm{~Hz}, \mathrm{CH}_{\mathrm{Ar}}\right), 7.28-7.37\left(\mathrm{~m}, 16 \mathrm{H}, \mathrm{CH}_{\mathrm{Ar}}\right), 7.53\left(\mathrm{~s}, 4 \mathrm{H}, \mathrm{CH}_{\mathrm{Ar}}\right)$. $\left.{ }^{13} \mathrm{C}_{\{}{ }^{1} \mathrm{H}\right\}$-NMR $\left(126 \mathrm{MHz}, \mathrm{CDCl}_{3}\right): \delta 46.9,51.4,126.8,126.9,127.6,127.7,128.4,128.7$, $128.9,136.0,136.6,137.4,171.3$.

HRMS (TOF MS ES+): Calcd for $\mathrm{C}_{36} \mathrm{H}_{33} \mathrm{~N}_{2} \mathrm{O}_{2}(\mathrm{M}+\mathrm{H})$ 525.2540. Found 525.2539.

\section{Conclusions}

Summing up, basing on the mechanism assumption, we described three new mechanism-guided copper-catalyzed protocols for the direct arylation of 2-bromo-2,2-difluoroacetamides using aryl boronic acids, aryl trialloxysilanes, and aryl sulphonium salts as the aryl donors. The deployment of the scope of the reactions showcased the unique tolerance of the developed methodologies towards vast range of structural patterns and substituents on all coupling parts. These methods offer rapid entry to structurally diverse aromatic amides from simple and commercially availed precursors. Noteworthily, all methodologies were prone for scale-up to gram quantities.

Supplementary Materials: Scheme S1: List of 2-bromo-2,2-difluoroacetamides 1 used for preparation of amides 5; Scheme S2: List of aryl boronic acids 2 used for preparation of amides 5; Scheme S3: List of (aryl)trialkoxysilanes 3 used for preparation of amides 5; Scheme S4: List of (aryl)dimethylsulfonium salts 4 used for preparation of amides 5 ; Copies ${ }^{1} \mathrm{H}$ and ${ }^{13} \mathrm{C}-\mathrm{NMR}$ spectra. 
Author Contributions: Conceptualization, V.O.I. and S.M.; methodology, S.M.; validation, S.M., M.J. and S.L.; investigation, S.M., M.J., S.L., V.O.I. and S.M.; writing-original draft preparation, V.O.I., S.M. and M.P.; writing—review and editing, V.O.I., S.M. and M.P.; visualization, V.O.I., S.M. and M.P.; supervision, V.O.I.; project administration, V.O.I., S.M. and M.P.; funding acquisition, V.O.I., S.M. and M.P. All authors have read and agreed to the published version of the manuscript.

Funding: This research was supported by a grant from National Science Centre (NSC) Poland within the framework of European Union's Horizon 2020 research and innovation program under the Marie Skłodowska-Curie grant agreement No. 665778 (POLONEZ 2 grant, Nr. 2016/21/P/ST5/00630) obtained by Satenik Mkrtchyan. This research project was also supported by a grant from National Science Centre (NSC) Poland, the SONATA 10 (Nr. 2015/19/D/ST5/02774) obtained by Viktor Iaroshenko. Viktor Iaroshenko also acknowledges the funding for his Visiting Professorship from the University of Salerno. Michael Pittelkow appreciates the support from the Danish Council for Independent Research (DFF 4181-00206 and 9040-00265) and from the University of Copenhagen.

Institutional Review Board Statement: Not applicable.

Informed Consent Statement: Not applicable.

Data Availability Statement: Data is contained within the article or Supplementary Materials.

Acknowledgments: Open access funding provided by University of Helsinki.

Conflicts of Interest: The authors declare no conflict of interest.

Sample Availability: Samples of all compounds are available from the authors.

\section{References}

1. Harrington, A.; Tal-Gan, Y. The Importance of Amide Protons in Peptide Drug Development. Available online: https://www. future-science.com/doi/abs/10.4155/fmc-2019-0238?journalCode=fmc (accessed on 8 November 2019).

2. Allen, C.L.; Williams, J.M. Metal-Catalysed Approaches to Amide Bond Formation. Chem. Soc. Rev. 2011, 40, 3405-3415. [CrossRef]

3. de Figueiredo, R.M.; Suppo, J.S.; Campagne, J.M. Nonclassical Routes for Amide Bond Formation. Chem. Rev. 2016, 116, 12029-12122. [CrossRef]

4. Valeur, E.; Bradley, M. Amide Bond Formation: Beyond the Myth of Coupling Reagents. Chem. Soc. Rev. 2009, 38, 606-631. [CrossRef] [PubMed]

5. Pattabiraman, V.R.; Bode, J.W. Rethinking Amide Bond Synthesis. Nature 2011, 480, 471-479. [CrossRef]

6. Ojeda-Porras, A.; Gamba-Sanchez, D. Recent Developments in Amide Synthesis Using Nonactivated Starting Materials. J. Org. Chem. 2016, 81, 11548-11555. [CrossRef] [PubMed]

7. Greenberg, A.; Breneman, C.M.; Liebman, J.F. The Amide Linkage: Structural Significance. In Chemistry, Biochemistry, and Materials Science; Wiley: Hoboken, NJ, USA, 2000.

8. Yun, S.H.; Ingole, P.G.; Choi, W.K.; Kim, J.H.; Lee, H.K. Synthesis of cross-linked amides and esters as thin film composite membrane materials yields permeable and selective material for water vapor/gas separation. J. Mater. Chem. A 2015, 3, 7888-7899. [CrossRef]

9. Atwood, J.L. Comprehensive Supramolecular Chemistry II; Elsevier Ltd.: Amsterdam, The Netherlands, 2017; 4568p.

10. Neri, P.; Sessler, J.L.; Wang, M.-X. Calixarenes and Beyond; Springer: Berlin/Heidelberg, Germany, 2016.

11. Urbach, A.R.; Ramalingam, V. Molecular Recognition of Amino Acids, Peptides, and Proteins by Cucurbit[n]uril Receptors. Isr. J. Chem. 2011, 51, 664-678. [CrossRef]

12. Zozulia, O.; Dolan, M.A.; Korendovych, I.V. Catalytic peptide assemblies. Chem. Soc. Rev. 2018, 47, 3621-3639. [CrossRef] [PubMed]

13. Metrano, A.J.; Miller, S.J. Peptide-Based Catalysts Reach the Outer Sphere through Remote Desymmetrization and Atroposelectivity. Acc. Chem. Res. 2019, 52, 199-215. [CrossRef]

14. Zabicky, J.; Patai, S. (Eds.) The Chemistry of the Amides, Part 1; Wiley: Hoboken, NJ, USA, 2010; ISBN 978-0-470-77123-5.

15. Chen, D.-H.; Sun, W.-T.; Zhu, C.-J.; Lu, G.-S.; Wu, D.-P.; Wang, A.-E.; Huang, P.-Q. Enantioselective Reductive Cyanation and Phosphonylation of Secondary Amides by Iridium and Chiral Thiourea Sequential Catalysis. Angew. Chem. Int. Ed. 2021, 60, 8827-8831. [CrossRef]

16. Tona, V.; Maryasin, B.; de la Torre, A.; Sprachmann, J.; González, L.; Maulide, N. Direct Regioselective Synthesis of Tetrazolium Salts by Activation of Secondary Amides under Mild Conditions. Org. Lett. 2017, 19, 2662-2665. [CrossRef] [PubMed]

17. Ou, W.; Huang, P.-Q. Amides as surrogates of aldehydes for C-C bond formation: Amide-based direct Knoevenagel-type condensation reaction and related reactions. Sci. China Chem. 2020, 63, 11-15. [CrossRef]

18. Takahashi, Y.; Sato, T.; Chida, N. Iridium-catalyzed Reductive Nucleophilic Addition to Tertiary Amides. Chem. Lett. 2019, 48, 1138-1141. [CrossRef] 
19. Chen, H.; Huang, Y.-H.; Ye, J.-L.; Huang, P.-Q. Double Addition of Alkynyllithium Reagents to Amides/Lactams: A Direct and Flexible Synthesis of 3-Amino-1,4-diynes Bearing an Aza-Quaternary Carbon Center. J. Org. Chem. 2019, 84, 9270-9281. [CrossRef] [PubMed]

20. Yoritate, M.; Takahashi, Y.; Tajima, H.; Ogihara, C.; Yokoyama, T.; Soda, Y.; Oishi, T.; Sato, T.; Chida, N. Unified Total Synthesis of Stemoamide-Type Alkaloids by Chemoselective Assembly of Five-Membered Building Blocks. J. Am. Chem. Soc. 2017, 139, 18386-18391. [CrossRef] [PubMed]

21. Dunetz, J.R.; Magano, J.; Weisenburger, G.A. Large-Scale Applications of Amide Coupling Reagents for the Synthesis of Pharmaceuticals. Org. Process Res. Dev. 2016, 20, 140-177. [CrossRef]

22. Montalbetti, C.A.G.N.; Falque, V. Amide Bond Formation and Peptide Coupling. Tetrahedron 2005, 61, 10827-10852. [CrossRef]

23. Sabatini, M.T.; Boulton, L.T.; Sneddon, H.F.; Sheppard, T.D. A green chemistry perspective on catalytic amide bond formation. Nat. Catal. 2019, 2, 10-17. [CrossRef]

24. Shangguan, N.; Katukojvala, S.; Greenberg, R.; Williams, L.J. The Reaction of Thio Acids with Azides: A New Mechanism and New Synthetic Applications. J. Am. Chem. Soc. 2003, 125, 7754-7755. [CrossRef]

25. Barnard, C.F.J. Palladium-Catalyzed Carbonylation-A Reaction Come of Age. Organometallics 2008, 27, 5402-5422. [CrossRef]

26. Friis, S.D.; Lindhardt, A.T.; Skrydstrup, T. The Development and Application of Two-Chamber Reactors and Carbon Monoxide Precursors for Safe Carbonylation Reactions. Acc. Chem. Res. 2016, 49, 594-605. [CrossRef]

27. Molander, G.A.; Wolfe, J.P.; Larhed, M. (Eds.) Science of Synthesis: Cross-Coupling and Heck-Type Reactions; Thieme: Stuttgart, Germany, 2013.

28. Liao, G.; Chen, H.M.; Shi, B.F. Synthesis of Phthalic Acid Derivatives via Pd-catalyzed Alkoxycarbonylation of Aromatic C-H Bonds with Alkyl Chloroformates. Chem. Commun. 2018, 54, 10859-10862. [CrossRef] [PubMed]

29. Diaba, F.; Montiel, J.A.; Serban, G.; Bonjoch, J. Synthesis of Normorphans through an Efficient Intramolecular Carbamoylation of Ketones. Org. Lett. 2015, 17, 3860-3863. [CrossRef]

30. Adachi, M.; Miyasaka, T.; Hashimoto, H.; Nishikawa, T. One-Step Transformation of Trichloroacetamide into Isonitrile. Org. Lett. 2017, 19, 380-383. [CrossRef]

31. Chen, J.; Guo, Y.P.; Sun, M.H.; Fan, G.T.; Zhou, L. Bromoform reaction of tertiary amines with $N, N$-dibromosulfonamides or NBS/sulfonamides. Chem. Commun. 2014, 50, 12367-12370. [CrossRef] [PubMed]

32. Ko, S.; Han, H.; Chang, S. Ru-Catalyzed Hydroamidation of Alkenes and Cooperative Aminocarboxylation Procedure with Chelating Formamide. Org. Lett. 2003, 2687-2690. [CrossRef] [PubMed]

33. Jo, Y.; Ju, J.; Choe, J.; Song, K.H.; Lee, S. The Scope and Limitation of Nickel-Catalyzed Aminocarbonylation of Aryl Bromides from Formamide Derivatives. J. Org. Chem. 2009, 74, 6358-6361. [CrossRef]

34. Iranpoor, N.; Panahi, F.; Roozbin, F.; Erfan, S.; Rahimi, S. Palladium-Catalyzed Aminocarbonylation of Aryl Halides with 2,4,6-Trichloro-1,3,5-triazine/Formamide Mixed Reagent. Eur. J. Org. Chem. 2016, 9, 1781-1787. [CrossRef]

35. Xiang, H.; Zhao, Q.; Tang, Z.; Xiao, J.; Xia, P.; Wang, C.; Yang, C.; Chen, X.; Yang, H. Visible-Light-Driven, Radical-Triggered Tandem Cyclization of o-Hydroxyaryl Enaminones: Facile Access to 3- $\mathrm{CF}_{2} / \mathrm{CF}_{3}-$ Containing Chromones. Org. Lett. 2017, 19, 146-149. [CrossRef] [PubMed]

36. An, L.; Xiao, Y.L.; Zhang, S.; Zhang, X.G. Bulky Diamine Ligand Promotes Cross-Coupling of Difluoroalkyl Bromides by Iron Catalysis. Angew. Chem. Int. Ed. 2018, 57, 6921-6925. [CrossRef]

37. Arlow, S.I.; Hartwig, J.F. Synthesis, Characterization, and Reactivity of Palladium Fluoroenolate Complexes. J. Am. Chem. Soc. 2017, 139, 16088-16091. [CrossRef] [PubMed]

38. Tao, N.; Wang, J.; Yuan, C.; Zeng, R.; Zhao, Y.-S. Palladium-Catalyzed Carboxylate-Assisted Ethoxycarboxylation of Aromatic Acids to Synthesize Monoethyl Phthalate Derivatives with Ethyl Bromodi fluoroacetate. Org. Lett. 2019, 21, 8607-8610. [CrossRef] [PubMed]

39. Ma, X.; Song, Q. Recent progress on selective deconstructive modes of halodifluoromethyl and trifluoromethyl-containing reagents. Chem. Soc. Rev. 2020, 49, 9197-9219. [CrossRef] [PubMed]

40. Santosh, K.; Thapa, G.S.; Vangala, A.S.; Giri, R. Copper-Catalyzed Hiyama Coupling of (Hetero)aryltriethoxysilanes with (Hetero)aryl Iodides. Org. Lett. 2013, 15, 5378-5381.

41. Gurung, S.K.; Thapa, S.; Kafle, A.; Dickie, D.A.; Giri, R. Copper-Catalyzed Suzuki-Miyaura Coupling of Arylboronate Esters: Transmetalation with (PN)CuF and Identification of Intermediates. Org. Lett. 2014, 16, 1264-1267. [CrossRef] [PubMed]

42. Gurung, S.K.; Thapa, S.; Shrestha, B.; Giri, R. Copper-catalysed cross-couplings of arylboronate esters with aryl and heteroaryl iodides and bromides. Org. Chem. Front. 2015, 2, 649-653. [CrossRef]

43. Zhu, L.; Le, L.; Yan, M.; Au, C.-T.; Qiu, R.; Kambe, N. Carbon-Carbon Bond Formation of Trifluoroacetyl Amides with Grignard Reagents via $\mathrm{C}(\mathrm{O})-\mathrm{CF}_{3}$ Bond Cleavage. J. Org. Chem. 2019, 84, 5635-5644. [CrossRef]

44. Pahl, J.; Brand, S.; Elsen, H.; Harder, S. Highly Lewis acidic cationic alkaline earth metal complexes. Chem. Commun. 2018, 54, 8685-8688. [CrossRef]

45. Harder, S.; Friedrich, A.; Pahl, J.; Eyselein, J.; Langer, J.; Hommes, N.V.E.; Görling, A. Magnesium-Halobenzene Bonding: Mapping the Halogen Sigma-Hole with a Lewis-Acidic Complex. Chem. Sci. 2021, 12, 2410-2418.

46. Majek, M.; von Wangelin, A.J. Mechanistic Perspectives on Organic Photoredox Catalysis for Aromatic Substitutions. Acc. Chem. Res. 2016, 49, 2316-2327. [CrossRef] 
47. Romero, N.A.; Nicewicz, D.A. Photoredox Catalysis for Building C-C Bonds from C(sp $\left.{ }^{2}\right)-H$ Bonds. Chem. Rev. 2016, 116, 10075-10166. [CrossRef] [PubMed]

48. Prier, C.K.; Rankic, D.A.; MacMillan, D.W.C. Visible Light Photoredox Catalysis with Transition Metal Complexes: Applications in Organic Synthesis. Chem. Rev. 2013, 113, 5322-5363. [CrossRef]

49. Lv, Y.; Pu, W.; Wang, Q.; Chen, Q.; Niu, J.; Zhang, Q. Copper-Catalyzed Aminodifluoroalkylation of Alkenes with $\alpha$ Bromodifluoroacetamides: Synthesis of 3,3-Difluoropyrrolidin-2-ones. Adv. Synth. Catal. 2017, 359, 3114-3119. [CrossRef]

50. Lv, Y.; Pu, W.; Chen, Q.; Wang, Q.; Niu, J.; Zhang, Q. Cu-Catalyzed Aminodifluoroalkylation of Alkynes and $\alpha-$ Bromodifluoroacetamides. J. Org. Chem. 2017, 82, 8282-8289. [CrossRef] [PubMed]

51. Zhang, M.; Li, W.; Duan, Y.; Xu, P.; Zhang, S.; Zhu, C. Cascade Photoredox/Iodide Catalysis: Access to Difluoro- $\gamma$-lactams via Aminodifluoroalkylation of Alkenes. Org. Lett. 2016, 18, 3266-3269. [CrossRef] [PubMed]

52. Ye, Z.; Gettys, K.E.; Shen, X.; Dai, M. Copper-Catalyzed Cyclopropanol Ring Opening Csp ${ }^{3}$-Csp ${ }^{3}$ Cross-Couplings with (Fluoro)Alkyl Halides. Org. Lett. 2015, 17, 6074-6077. [CrossRef] [PubMed]

53. Wei, X.-J.; Wang, L.; Du, S.-F.; Wub, L.-Z.; Liu, Q. Visible-light photoredox intramolecular difluoroacetamidation: Facile synthesis of 3,3-difluoro-2-oxindoles from bromodifluoroacetamides. Org. Biomol. Chem. 2016, 14, 2195-2199. [CrossRef]

54. Lermontov, S.A.; Polivanova, A.G.; Shkavrov, S.B. Interaction of $\alpha, \alpha$-difluoroazides with trivalent phosphorus compounds and triphenylantimony. Russ. J. Gen. Chem. 2010, 80, 1646-1651. [CrossRef]

55. Deng, S.; Chen, H.; Ma, X.; Zhou, Y.; Yang, K.; Lan, Y.; Song, Q. S8-Catalyzed triple cleavage of bromodifluoro compounds for the assembly of N-containing heterocycles. Chem. Sci. 2019, 10, 6828-6833. [CrossRef]

56. Chen, C.; Zeng, R.; Zhang, J.; Zhao, Y. Ruthenium-Catalyzed Difluoroalkylation of 8-Aminoquinoline Amides at the C5-Position. Eur. J. Org. Chem. 2017, 10, 6947-6950. [CrossRef]

57. Ye, Z.-P.; Xia, P.-J.; Liu, F.; Hu, Y.-Z.; Song, D.; Xiao, J.-A.; Huang, P.; Xiang, H.-Y.; Chen, X.-Q.; Yang, H. Visible-Light-Induced, Catalyst-Free Radical Cross-Coupling Cyclization of $N$-Allylbromodifluoroacetamides with Disulfides or Diselenides. J. Org. Chem. 2020, 85, 5670-5682. [CrossRef] [PubMed]

58. Lu, M.-Z.; Ding, X.; Shao, C.; Hu, Z.; Luo, H.; Zhi, S.; Hu, H.; Kan, Y.; Loh, T.-P. Direct Hiyama Cross-Coupling of (Hetero)arylsilanes with $\mathrm{C}\left(\mathrm{sp}^{2}\right)-\mathrm{H}$ Bonds Enabled by Cobalt Catalysis. Org. Lett. 2020, 22, 2663-2668. [CrossRef]

59. Wang, Z.; Chang, S. Copper-Mediated Transformation of Organosilanes to Nitriles with DMF and Ammonium Iodide. Org. Lett. 2013, 15, 1990-1993. [CrossRef] [PubMed]

60. Luo, H.; Xie, Q.; Sun, K.; Deng, J.; Xu, L.; Wang, K.; Luo, X. Rh(iii)-catalyzed C-7 arylation of indolines with arylsilanes via C-H activation. RSC Adv. 2019, 9, 18191-18195. [CrossRef]

61. Varenikov, A.; Gandelman, M. Synthesis of chiral $\alpha$-trifluoromethyl alcohols and ethers via enantioselective Hiyama crosscouplings of bisfunctionalized electrophiles. Nat. Commun. 2018, 9, 3566. [CrossRef]

62. Lu, M.-Z.; Luo, H.; Hu, Z.; Shao, C.; Kan, Y.; Loh, T.-P. Directed Palladium(II)-Catalyzed Intermolecular Anti-Markovnikov Hydroarylation of Unactivated Alkenes with (Hetero)arylsilanes. Org. Lett. 2020, 22, 9022-9028. [CrossRef]

63. Nguyen, T.V.Q.; Yoo, W.-J.; Kobayashi, S. Copper-Catalyzed Carboxylation of Aryl- and Alkenyltrialkoxysilanes. Asian J. Org. Chem. 2018, 7, 116-118. [CrossRef]

64. von der Lehr, M.; Ellinghaus, R.; Smarsly, B.M. Hierarchically porous monolithic silica with varying porosity using bis(trimethoxysilyl)arenes as precursors. New J. Chem. 2016, 40, 4455-4463. [CrossRef]

65. Huang, C.; Feng, J.; Ma, R.; Fang, S.; Lu, T.; Tang, W.; Du, D.; Gao, J. Redox-Neutral Borylation of Aryl Sulfonium Salts via C-S Activation Enabled by Light. Org. Lett. 2019, 21, 9688-9692. [CrossRef]

66. Yanagi, T.; Somerville, R.J.; Nogi, K.; Martin, R.; Yorimitsu, H. Ni-Catalyzed Carboxylation of C(sp $\left.{ }^{2}\right)-S$ Bonds with $\mathrm{CO}_{2}$ : Evidence for the Multifaceted Role of Zn. ACS Catal. 2020, 10, 2117-2123. [CrossRef]

67. Uno, D.; Minami, H.; Otsuka, S.; Nogi, K.; Yorimitsu, H. Palladium-Catalyzed Mizoroki-Heck-Type Alkenylation of Monoaryldialkylsulfoniums. Chem. Asian J. 2018, 13, 2397-2400.

68. Sutariya, P.G.; Soni, H.; Gandhi, S.A.; Pandya, A. Luminescent behavior of pyrene-allied calix[4]arene for the highly pH-selective recognition and determination of $\mathrm{Zn}^{2+}, \mathrm{Hg}^{2+}$ and $\mathrm{I}^{-}$via the CHEF-PET mechanism: Computational experiment and paper-based device. New J. Chem. 2019, 43, 9855-9864. [CrossRef]

69. Mendoza-Espinosa, D.; Martinez-Ortega, A.B.; Quiroz-Guzman, M.; Golen, J.A.; Rheingold, L.A.; Hanna, T.A. Synthesis, structures and full characterization of $p$-tert-butylcalix[5]arene mono-, di-, tri- and pentaanionic ligand precursors. J. Organomet. Chem. 2009, 694, 1509-1523. [CrossRef] 